\title{
Cooperative P-H Bond Activation with Ruthenium and Iridium Carbene Complexes
}

\author{
Julia Weismann, Lennart T. Scharf and Viktoria H. Gessner*
}

Institut für Anorganische Chemie, Julius-Maximilians-Universität Würzburg, Am Hubland, 97074 Würzburg, Germany.

Present address: Lehrstuhl für Anorganische Chemie II, Ruhr-Universität Bochum, Universitätsstraße 150, D-44780 Bochum, Germany.

\section{Index}

1. Synthetic procedure for the $\mathrm{P}-\mathrm{H}$ bond activation in phosphines, NMR data and spectra of the reaction mixtures

2. ${ }^{1} \mathrm{H},{ }^{13} \mathrm{C}$ and ${ }^{31} \mathrm{P}$ NMR spectra of the isolated compounds $\quad \mathrm{S} 8$

$\begin{array}{ll}\text { 3. Crystal Structure Determination } & \text { S17 }\end{array}$

3.1 Crystal Structure Determination of $\mathbf{4 a} \quad$ S18

3.2 Crystal Structure Determination of $\mathbf{4 b} \quad$ S21

$\begin{array}{ll}\text { 3.3 Crystal Structure Determination of 5a } & \text { S24 }\end{array}$

3.4 Crystal Structure Determination of $\mathbf{5 b} \quad$ S26

4. Computational Details $\quad$ S30

$\begin{array}{lr}\text { 4.1. Thermodynamics of different isomers } & \text { S30 }\end{array}$

$\begin{array}{lr}\text { 4.2 Mechanistic Studies } & \text { S42 }\end{array}$ 


\section{Synthesis and Characterization of Complexes 3a-3f.}

General procedure: In a $J$. Young NMR tube, $30.0 \mathrm{mg}(49.5 \mu \mathrm{mol})$ of ruthenium carbene complex 1 were dissolved in $0.7 \mathrm{~mL}$ dry $\mathrm{C}_{6} \mathrm{D}_{6}$ (for reactions at room temperature) or dry toluene (for reactions at $\left.-78{ }^{\circ} \mathrm{C}\right)$ and the purple solution was treated with one equivalent $(49.5 \mu \mathrm{mol})$ of the appropriate phosphine. Subsequently, a color change to red/orange occurred, and the reaction mixture was screened by ${ }^{31} \mathrm{P}\left\{{ }^{1} \mathrm{H}\right\}$ NMR spectroscopy, indicating a complete conversion of the starting materials. Thus, the NMR spectroscopic studies confirmed the presence of more than one main product and consequently the desired activation products $\mathbf{3 a - 3 f}$ could not be isolated and fully characterized. ${ }^{1} \mathrm{H}$ and ${ }^{31} \mathrm{P}\left\{{ }^{1} \mathrm{H}\right]$ NMR data were determined out of the spectra of the reaction mixtures.

\section{${ }^{31} \mathbf{P}\left\{{ }^{1} \mathrm{H}\right\}$ and ${ }^{1} \mathbf{H}$ NMR data of Complexes $3 \mathrm{~b}-3 \mathrm{f}$.}

3b: ${ }^{1} \mathrm{H}$ NMR $\left(400.1 \mathrm{MHz}, \mathrm{C}_{6} \mathrm{D}_{6}\right): \delta=1.06\left(\mathrm{~d},{ }^{3} J_{\mathrm{HH}}=6.99 \mathrm{~Hz}, 3 \mathrm{H} ; \mathrm{CH}\left(\mathrm{CH}_{3}\right)_{2}\right), 1.16\left(\mathrm{~d},{ }^{3} J_{\mathrm{HH}}=6.94\right.$ $\mathrm{Hz}, 3 \mathrm{H} ; \mathrm{CH}\left(\mathrm{CH}_{3}\right)_{2}$ ), 1.60 (s, $3 \mathrm{H} ; \mathrm{CH}_{3}$ ), 2.88-2.98 (sept., $\left.{ }^{3} J_{\mathrm{HH}}=6.77 \mathrm{~Hz}, 1 \mathrm{H} ; \mathrm{CH}\left(\mathrm{CH}_{3}\right)_{2}\right), 3.56$ (dd, $\left.{ }^{2} J_{\mathrm{PH}}=10.5,{ }^{3} J_{\mathrm{PH}}=4.18 \mathrm{~Hz}, 1 \mathrm{H} ; \mathrm{PCHS}\right), 5.09\left(\mathrm{~d},{ }^{3} J_{\mathrm{HH}}=5.24 \mathrm{~Hz}, 1 \mathrm{H} ; \mathrm{CH}_{\text {Cymene }}\right), 5.27\left(\mathrm{~d},{ }^{3} J_{\mathrm{HH}}=5.82\right.$ $\mathrm{Hz}, 1 \mathrm{H}$; $\left.\mathrm{CH}_{\text {Cymene }}\right), 5.38$ (br., $2 \mathrm{H}$; $\left.\mathrm{CH}_{\text {Cymene }}\right), 6.16-8.32\left(\mathrm{CH}_{\text {arom. }}\right) .{ }^{31} \mathrm{P}\left\{{ }^{1} \mathrm{H}\right\} \mathrm{NMR}\left(162.0 \mathrm{MHz}, \mathrm{C}_{6} \mathrm{D}_{6}\right): \delta$ $=39.0\left(\mathrm{~d},{ }^{3} J_{\mathrm{PP}}=2.89 \mathrm{~Hz}\right), 56.7\left(\mathrm{~d},{ }^{3} J_{\mathrm{PP}}=3.74 \mathrm{~Hz} ; P \mathrm{~S}\right)$.

3c: ${ }^{1} \mathrm{H}$ NMR (400.1 MHz, $\left.\mathrm{C}_{6} \mathrm{D}_{6}\right): \delta=1.00\left(\mathrm{~d},{ }^{3} J_{\mathrm{HH}}=7.00 \mathrm{~Hz}, 3 \mathrm{H} ; \mathrm{CH}\left(\mathrm{CH}_{3}\right)_{2}\right), 1.18\left(\mathrm{~d},{ }^{3} J_{\mathrm{HH}}=6.81\right.$ $\mathrm{Hz}, 3 \mathrm{H} ; \mathrm{CH}\left(\mathrm{CH}_{3}\right)_{2}$ ), 1.63 (s, $3 \mathrm{H} ; \mathrm{CH}_{3}$ ), 2.70-2.80 (sept., $\left.{ }^{3} J_{\mathrm{HH}}=7.00 \mathrm{~Hz}, 1 \mathrm{H} ; \mathrm{CH}\left(\mathrm{CH}_{3}\right)_{2}\right), 3.61$ (dd, $\left.{ }^{2} J_{\mathrm{PH}}=10.1,{ }^{3} J_{\mathrm{PH}}=4.21 \mathrm{~Hz}, 1 \mathrm{H} ; \mathrm{PCHS}\right), 5.07\left(\mathrm{~d},{ }^{3} J_{\mathrm{HH}}=5.88 \mathrm{~Hz}, 1 \mathrm{H} ; \mathrm{C} H_{\text {Cymene }}\right), 5.19\left(\mathrm{~d},{ }^{3} J_{\mathrm{HH}}=5.70\right.$ $\mathrm{Hz}, 1 \mathrm{H}$; $\left.\mathrm{CH}_{\text {Cymene }}\right), 5.25$ (d, ${ }^{3} J_{\mathrm{HH}}=5.76 \mathrm{~Hz}, 1 \mathrm{H}$; $\left.\mathrm{CH}_{\text {Cymene }}\right), 5.56\left(\mathrm{~d},{ }^{3} J_{\mathrm{HH}}=5.95 \mathrm{~Hz}, 1 \mathrm{H} ; \mathrm{CH}_{\text {Cymene }}\right)$, 6.57-6.75 (m, $8 \mathrm{H}$; $\mathrm{CH}_{\text {arom. }}$ ), 7.03-7.11 (m, $5 \mathrm{H}$; $\left.\mathrm{CH}_{\text {arom. }}\right), 7.52-7.54\left(\mathrm{~m}, 2 \mathrm{H} ; \mathrm{CH}_{\text {arom. }}\right), 7.83$ (dd, $J=$ $1.90,4.36 \mathrm{~Hz}, 4 \mathrm{H}$; $\left.\mathrm{CH}_{\text {arom. }}\right), 8.15-8.21\left(\mathrm{~m}, 2 \mathrm{H} ; \mathrm{C} H_{\mathrm{P}(\mathrm{S}) \mathrm{Ph}, \text { ortho. }}{ }^{31} \mathrm{P}\left\{{ }^{1} \mathrm{H}\right\} \mathrm{NMR}\left(162.0 \mathrm{MHz}, \mathrm{C}_{6} \mathrm{D}_{6}\right): \delta=\right.$ 35.5 (br.), 59.5 (d, $\left.{ }^{3} J_{\mathrm{PP}}=14.8 \mathrm{~Hz} ; P \mathrm{~S}\right)$.

3d: ${ }^{1} \mathrm{H}$ NMR (400.1 MHz, $\left.\mathrm{C}_{6} \mathrm{D}_{6}\right): \delta=0.79\left(\mathrm{~d},{ }^{3} J_{\mathrm{HH}}=6.90 \mathrm{~Hz}, 3 \mathrm{H}\right.$; $\left.\mathrm{CH}\left(\mathrm{CH}_{3}\right)_{2}\right), 1.03\left(\mathrm{~d},{ }^{3} J_{\mathrm{HH}}=6.91\right.$ $\mathrm{Hz}, 3 \mathrm{H} ; \mathrm{CH}\left(\mathrm{CH}_{3}\right)_{2}$ ), 1.84 (s, $3 \mathrm{H} ; \mathrm{CH}_{3}$ ), 2.68-2.78 (sept., $\left.{ }^{3} J_{\mathrm{HH}}=6.85 \mathrm{~Hz}, 1 \mathrm{H} ; \mathrm{CH}\left(\mathrm{CH}_{3}\right)_{2}\right), 3.17$ (s, 3 $\left.\mathrm{H} ; \mathrm{OCH}_{3}\right), 3.24\left(\mathrm{~s}, 3 \mathrm{H} ; \mathrm{OCH}_{3}\right), 3.86\left(\mathrm{dd},{ }^{2} J_{\mathrm{PH}}=8.57,{ }^{3} J_{\mathrm{PH}}=6.87 \mathrm{~Hz}, 1 \mathrm{H} ; \mathrm{PCHS}\right), 5.47\left(\mathrm{~d},{ }^{3} J_{\mathrm{HH}}=5.86\right.$ $\mathrm{Hz}, 1 \mathrm{H}$; CH $\left.H_{\text {Cymene }}\right), 5.65$ (d, ${ }^{3} J_{\mathrm{HH}}=6.18 \mathrm{~Hz}, 1 \mathrm{H}$; $\left.\mathrm{C}_{\text {Cymene }}\right), 6.23$ (d, $\left.{ }^{3} J_{\mathrm{HH}}=5.59 \mathrm{~Hz}, 1 \mathrm{H} ; \mathrm{CH}_{\text {Cymene }}\right)$, 6.46-8.09 (CH $\left.\mathrm{C}_{\text {arom. }}\right) .{ }^{31} \mathrm{P}\left\{{ }^{1} \mathrm{H}\right\} \mathrm{NMR}\left(162.0 \mathrm{MHz}, \mathrm{C}_{6} \mathrm{D}_{6}\right): \delta=36.5\left(\mathrm{~d},{ }^{3} J_{\mathrm{PP}}=2.95 \mathrm{~Hz} ; P \mathrm{O}\right), 56.6\left(\mathrm{~d},{ }^{3} J_{\mathrm{PP}}=\right.$ $3.75 \mathrm{~Hz} ; P S)$.

3e: ${ }^{1} \mathrm{H}$ NMR $\left(400.1 \mathrm{MHz}, \mathrm{C}_{6} \mathrm{D}_{6}\right): \delta=0.83\left(\mathrm{~d},{ }^{3} J_{\mathrm{HH}}=6.91 \mathrm{~Hz}, 3 \mathrm{H}\right.$; $\left.\mathrm{CH}\left(\mathrm{CH}_{3}\right)_{2}\right), 1.29\left(\mathrm{~d},{ }^{3} J_{\mathrm{HH}}=6.80\right.$

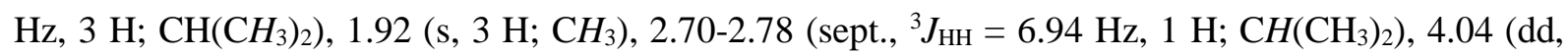
$\left.{ }^{2} J_{\mathrm{PH}}=8.36,{ }^{3} J_{\mathrm{PH}}=6.71 \mathrm{~Hz}, 1 \mathrm{H} ; \mathrm{PCHS}\right), 5.57\left(\mathrm{~d},{ }^{3} J_{\mathrm{HH}}=6.05 \mathrm{~Hz}, 1 \mathrm{H} ; \mathrm{CH}_{\text {Cymene }}\right), 5.72\left(\mathrm{~d},{ }^{3} J_{\mathrm{HH}}=6.32\right.$ $\mathrm{Hz}, 1 \mathrm{H}$; $\left.\mathrm{CH}_{\text {Cymene }}\right), 5.96\left(\mathrm{dd},{ }^{3} J_{\mathrm{HH}}=2.23,9.06 \mathrm{~Hz}, 1 \mathrm{H}\right.$; $\left.\mathrm{CH}_{\text {Cymene }}\right), 6.21-8.40\left(\mathrm{CH}_{\text {arom. }}\right) .{ }^{31} \mathrm{P}\left\{{ }^{1} \mathrm{H}\right\} \mathrm{NMR}$ (162.0 MHz, $\left.\mathrm{C}_{6} \mathrm{D}_{6}\right): \delta=34.3$ (br.), 56.5 (d, ${ }^{3} J_{\mathrm{PP}}=3.90 \mathrm{~Hz} ; P S$ ).

3f: ${ }^{1} \mathrm{H}$ NMR $\left(400.1 \mathrm{MHz}, \mathrm{C}_{6} \mathrm{D}_{6}\right): \delta=0.96\left(\mathrm{~d},{ }^{3} J_{\mathrm{HH}}=6.97 \mathrm{~Hz}, 3 \mathrm{H} ; \mathrm{CH}\left(\mathrm{CH}_{3}\right)_{2}\right), 1.01\left(\mathrm{~d},{ }^{3} J_{\mathrm{HH}}=6.84\right.$ $\mathrm{Hz}, 3 \mathrm{H} ; \mathrm{CH}\left(\mathrm{CH}_{3}\right)_{2}$ ), 1.63 (s, $3 \mathrm{H} ; \mathrm{CH}_{3}$ ), 2.54-2.64 (sept., $\left.{ }^{3} J_{\mathrm{HH}}=6.88 \mathrm{~Hz}, 1 \mathrm{H} ; \mathrm{CH}\left(\mathrm{CH}_{3}\right)_{2}\right), 3.90$ (dd, $\left.{ }^{2} J_{\mathrm{PH}}=9.29,{ }^{3} J_{\mathrm{PH}}=2.49 \mathrm{~Hz}, 1 \mathrm{H} ; \mathrm{PCHS}\right), 4.92\left(\mathrm{~d},{ }^{3} J_{\mathrm{HH}}=5.80 \mathrm{~Hz}, 1 \mathrm{H} ; \mathrm{CH}_{\text {Cymene }}\right), 4.97\left(\mathrm{~d},{ }^{3} J_{\mathrm{HH}}=5.68\right.$ $\left.\mathrm{Hz}, 1 \mathrm{H} ; \mathrm{CH}_{\text {Cymene }}\right), 5.20\left(\mathrm{~d},{ }^{3} J_{\mathrm{HH}}=5.80 \mathrm{~Hz}, 1 \mathrm{H} ; \mathrm{CH}_{\text {Cymene }}\right), 5.83\left(\mathrm{~d},{ }^{3} J_{\mathrm{HH}}=5.99 \mathrm{~Hz}, 1 \mathrm{H} ; \mathrm{C}_{\text {Cymene }}\right)$, 6.50-6.55 (m, $2 \mathrm{H}$; $\mathrm{CH}_{\text {arom }}$ ), 6.61-6.74 (m, $\left.6 \mathrm{H} ; \mathrm{CH}_{\text {arom. }}\right), 7.02-7.10\left(\mathrm{~m}, 3 \mathrm{H} ; \mathrm{C} H_{\text {arom }}\right), 7.40$ (d, ${ }^{2} J_{\mathrm{HH}}=$ $7.19 \mathrm{~Hz}, 2 \mathrm{H}$; $\left.\mathrm{CH}_{\text {arom }}\right), 7.64$ (s, $2 \mathrm{H}$; $\left.\mathrm{CH}_{\text {arom }}\right), 7.99-8.06$ (m, $\left.2 \mathrm{H} ; \mathrm{CH}_{\text {arom }}\right), 8.40$ (d, ${ }^{3} J_{\mathrm{HH}}=3.35 \mathrm{~Hz}, 4 \mathrm{H}$; $\left.\mathrm{CH}_{\text {arom. }}\right){ }^{31} \mathrm{P}\left\{{ }^{1} \mathrm{H}\right\}$ NMR $\left(162.0 \mathrm{MHz}, \mathrm{C}_{6} \mathrm{D}_{6}\right): \delta=24.4$ (br.), $62.8\left(\mathrm{~d},{ }^{3} J_{\mathrm{PP}}=14.0 \mathrm{~Hz} ; P \mathrm{~S}\right)$. 
7ach Zugabe Phosphan
APROTON C6D6 $\{$ :ITopspin21\} User 30

Current Data Parameters
NAME JUB877a 30290 EXPNO

F2- Acquisition Parameter
Date
20160129

$\begin{array}{ll}\text { Date } & 20160129 \\ \text { Time } & 12.30 \\ \text { TIST } & 12\end{array}$

INSTRUM $5 \mathrm{mpect}$

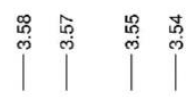

TD PROG 99336

SOLVENT ${ }_{16}^{99336}$

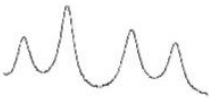

IIDRES

$0.083335 \mathrm{~Hz}$
AQ
$5.9999442 \mathrm{sec}$

90.5
60.400 use

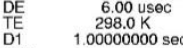

$\begin{array}{ll}\text { TDO } & 1 \\ \text { NUC1 } & 10\end{array}$

\begin{tabular}{ll} 
PI & 10.30 usec \\
PLW1 & -1.00000000 \\
\hline
\end{tabular}

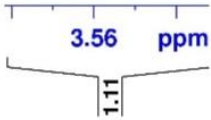

-

F2- Processing parameter
SS 131072 .
SF $400.130049 \mathrm{MHz}$
WDW
WM

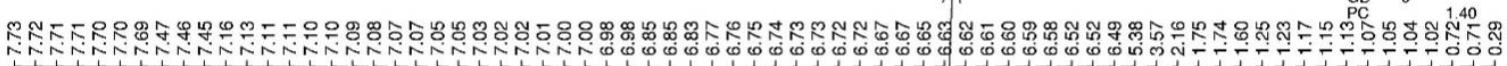
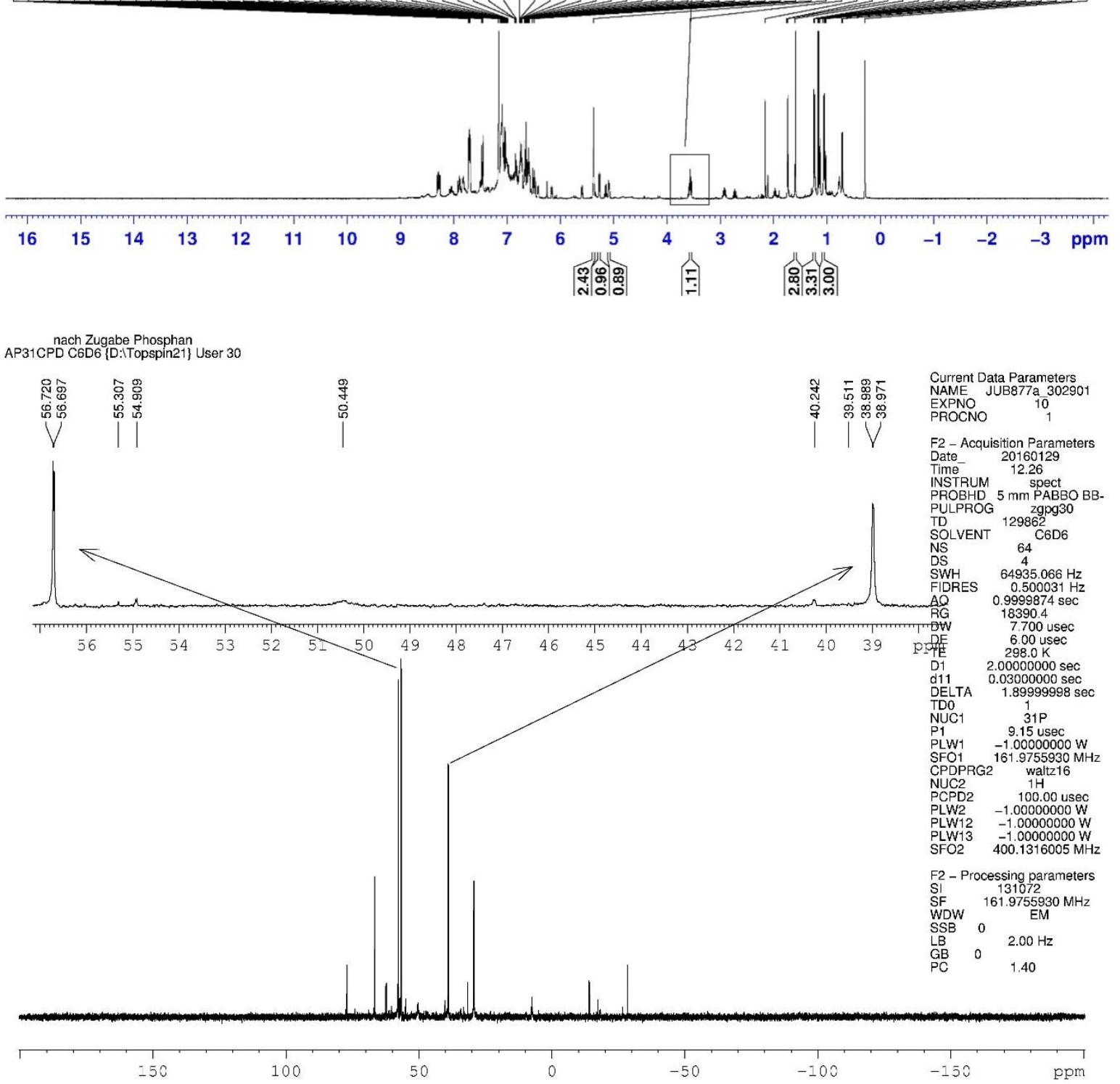

Figure S1. ${ }^{1} \mathrm{H}$ and ${ }^{31} \mathrm{P}\left\{{ }^{1} \mathrm{H}\right\}$ NMR spectra of $\mathbf{3 b}$ in $\mathrm{C}_{6} \mathrm{D}_{6}$. 


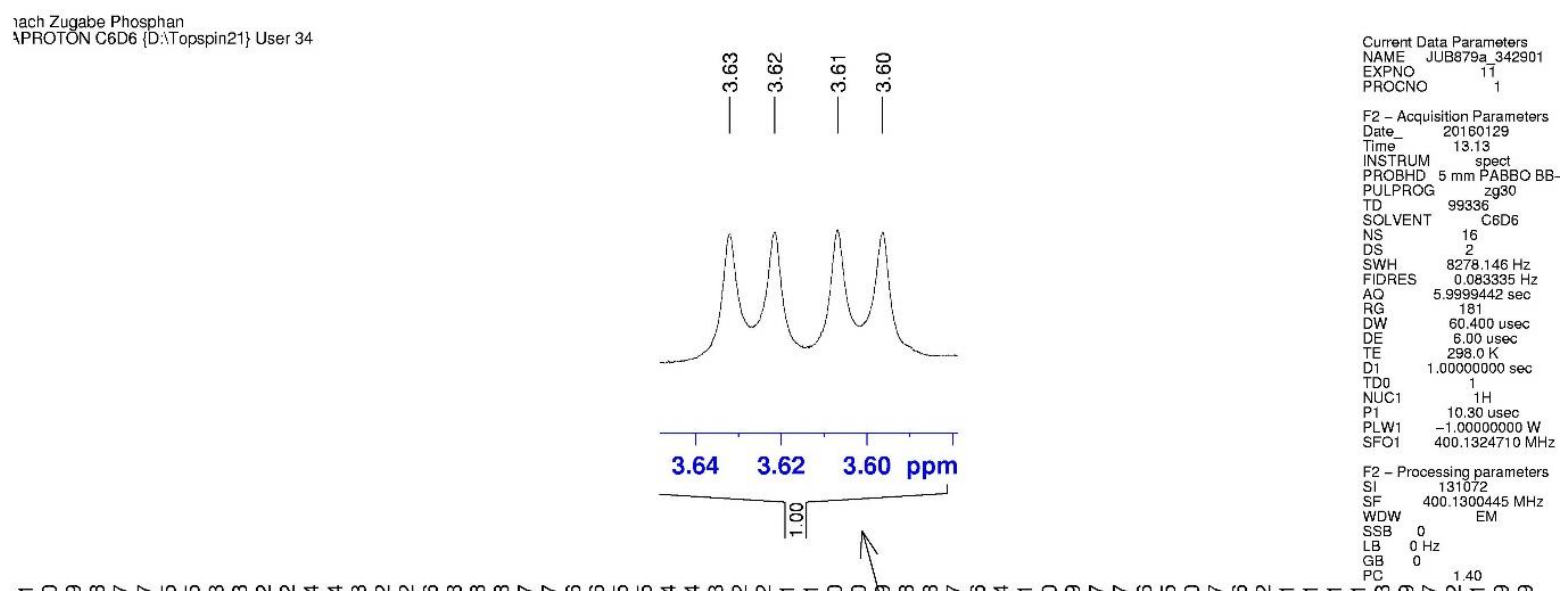

ำำ

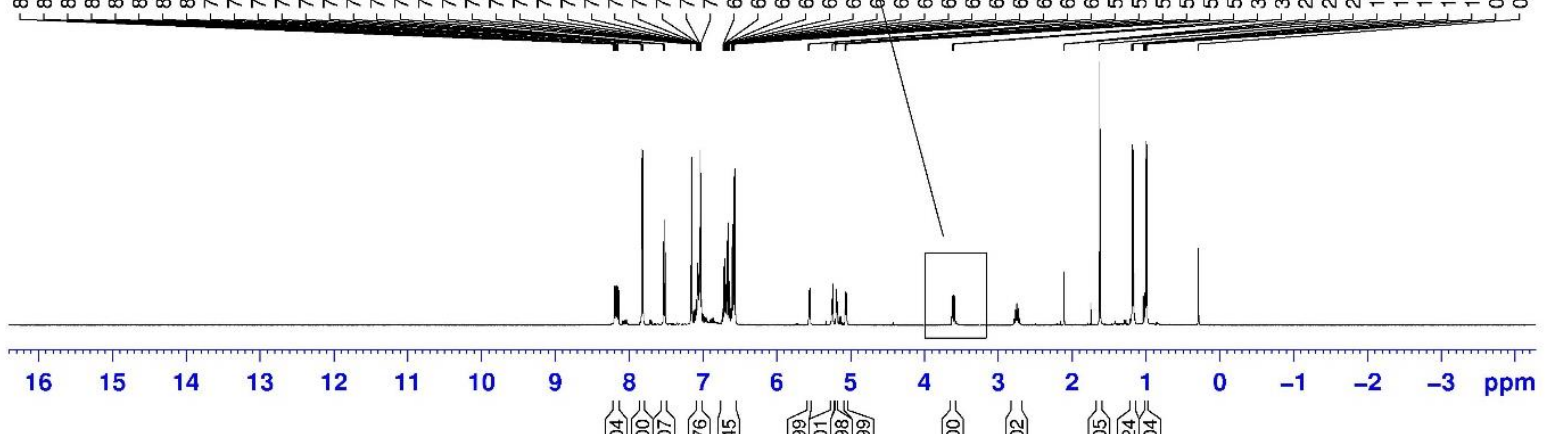

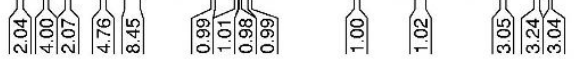

nach Zugabe Phosphan
AP31CPD C6D $\{$ D:TTopspin21\} User 34

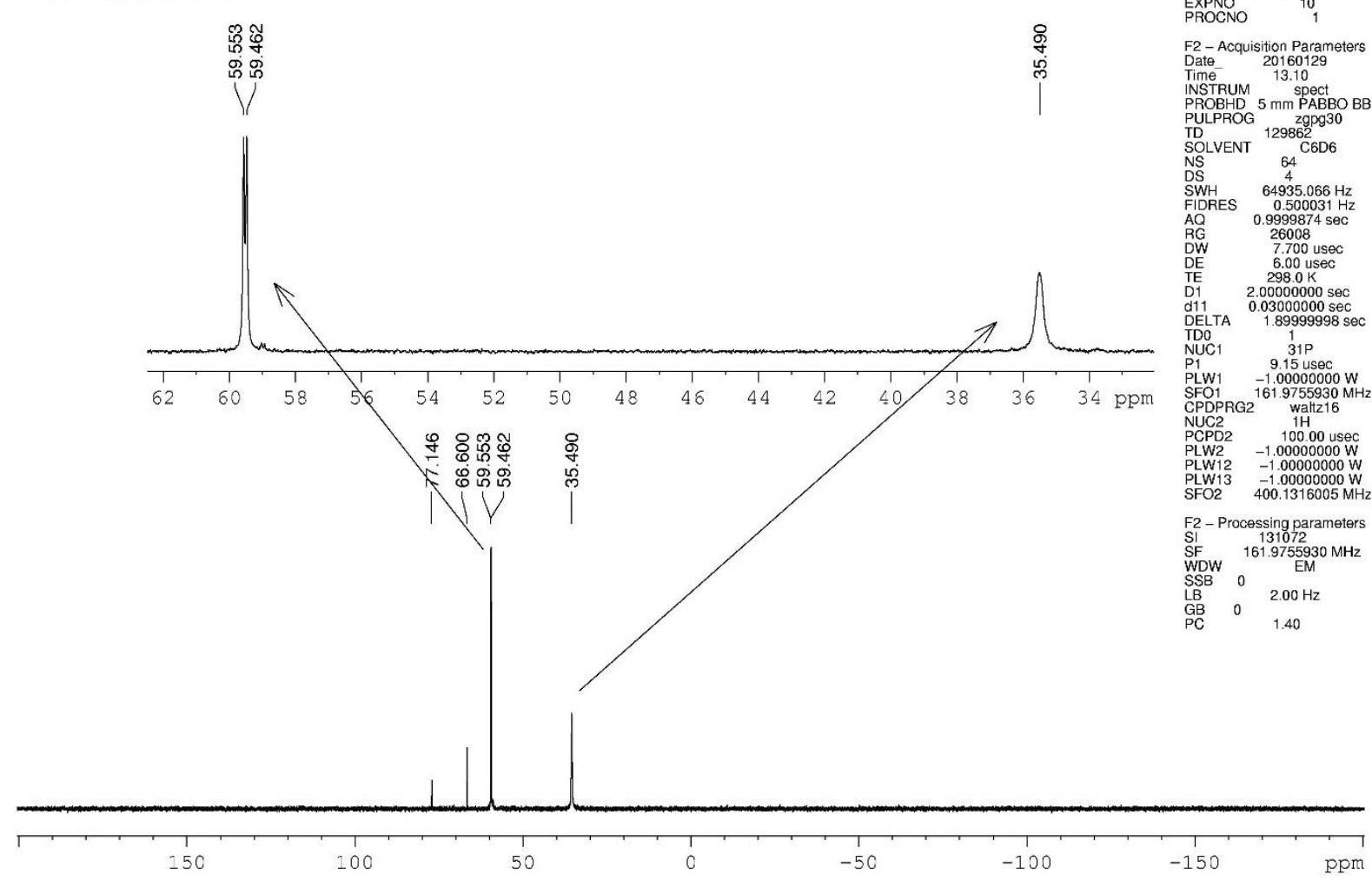

Figure S2. ${ }^{1} \mathrm{H}$ and ${ }^{31} \mathrm{P}\left\{{ }^{1} \mathrm{H}\right\}$ NMR spectra of $3 \mathbf{c}$ in $\mathrm{C}_{6} \mathrm{D}_{6}$. 
Current Data Parameters
NAME JUB880 100102

EXPNO
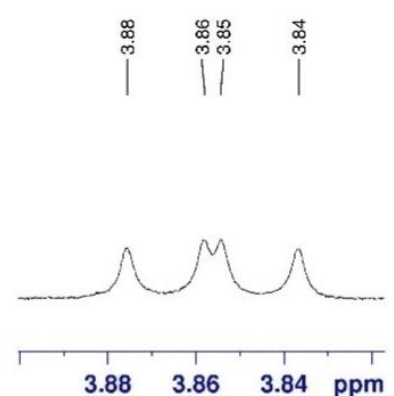

$\begin{array}{llll}3.88 & 3.86 & 3.84 & \mathrm{ppm}\end{array}$

]?:-

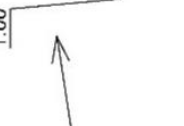

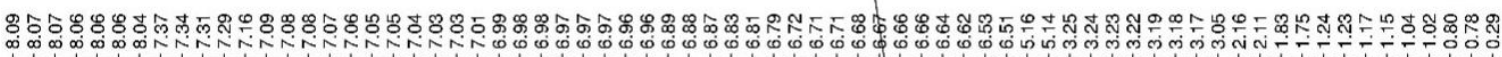
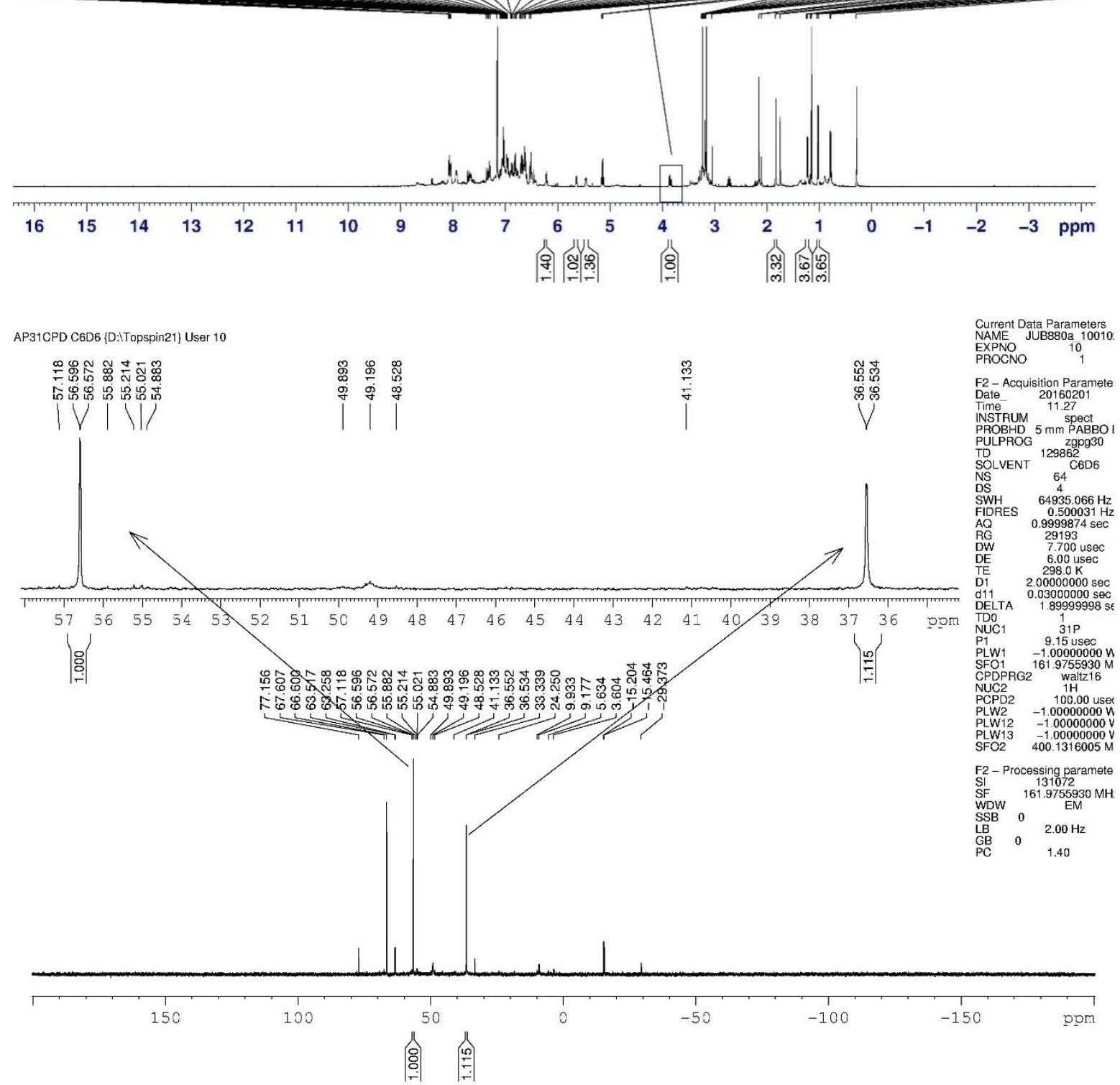

Figure S3. ${ }^{1} \mathrm{H}$ and ${ }^{31} \mathrm{P}\left\{{ }^{1} \mathrm{H}\right\}$ NMR spectra of $\mathbf{3 d}$ in $\mathrm{C}_{6} \mathrm{D}_{6}$. 
nach Zugabe Phosphan
APROTON C6D6 \{D:ITopspin21\} User 31

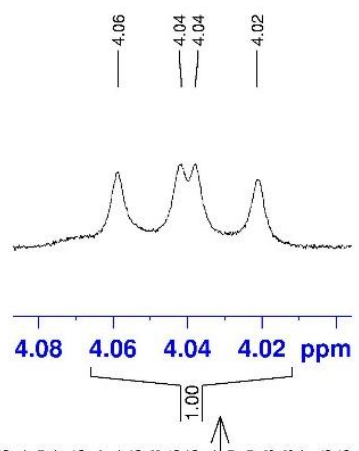

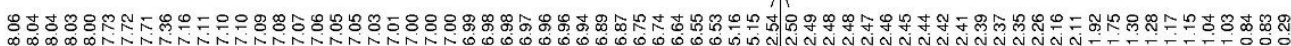
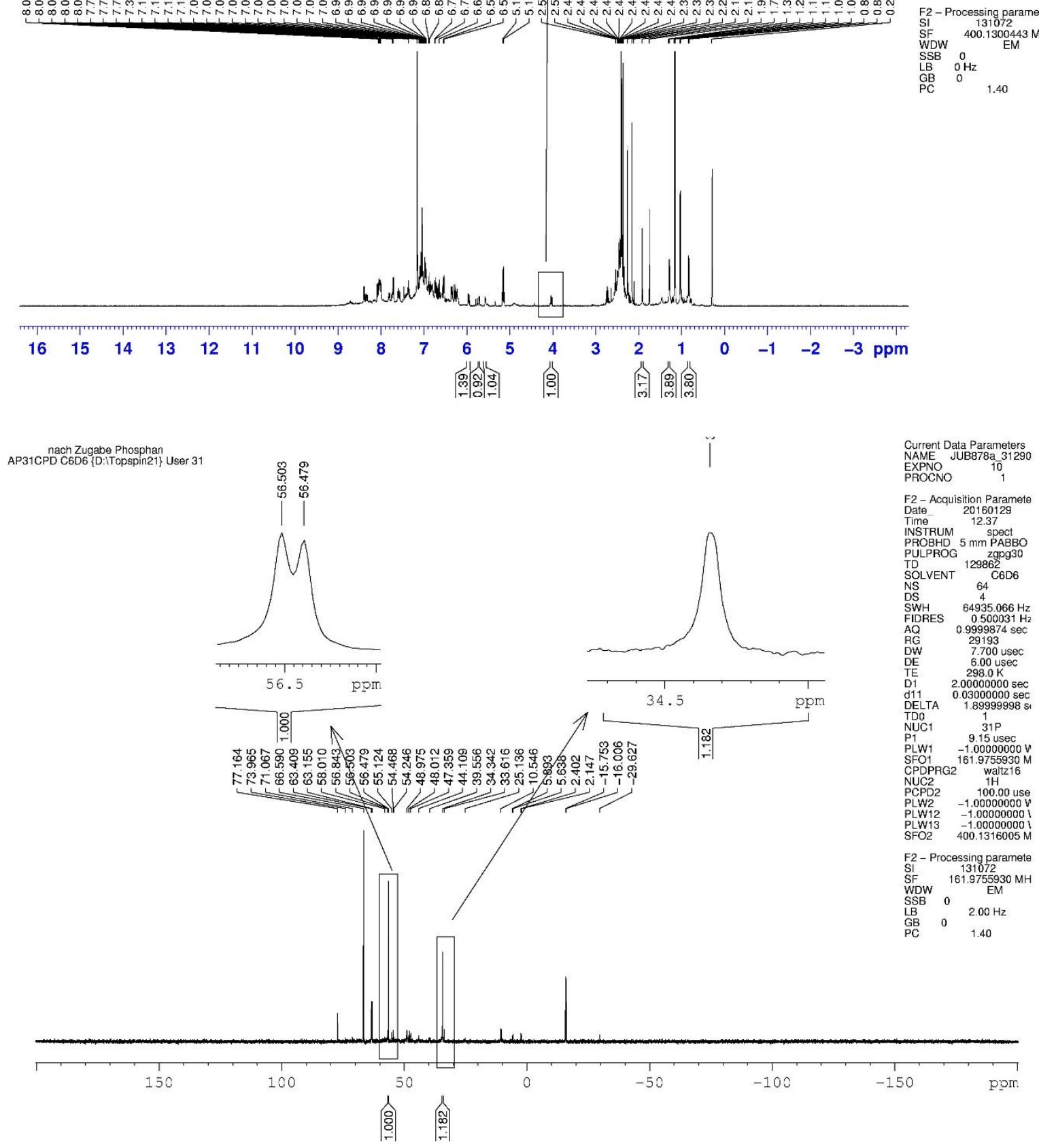

Figure S4. ${ }^{1} \mathrm{H}$ and ${ }^{31} \mathrm{P}\left\{{ }^{1} \mathrm{H}\right\}$ NMR spectra of $3 e$ in $\mathrm{C}_{6} \mathrm{D}_{6}$. 


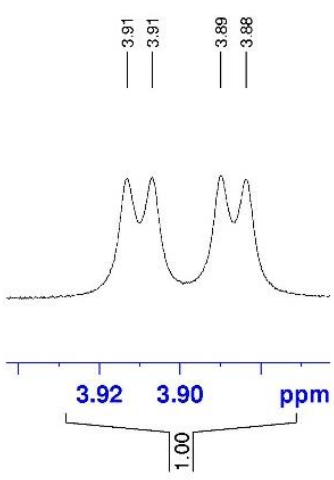

$\wedge$

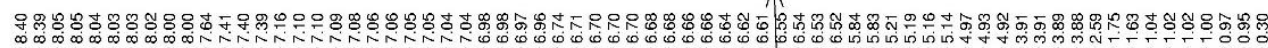

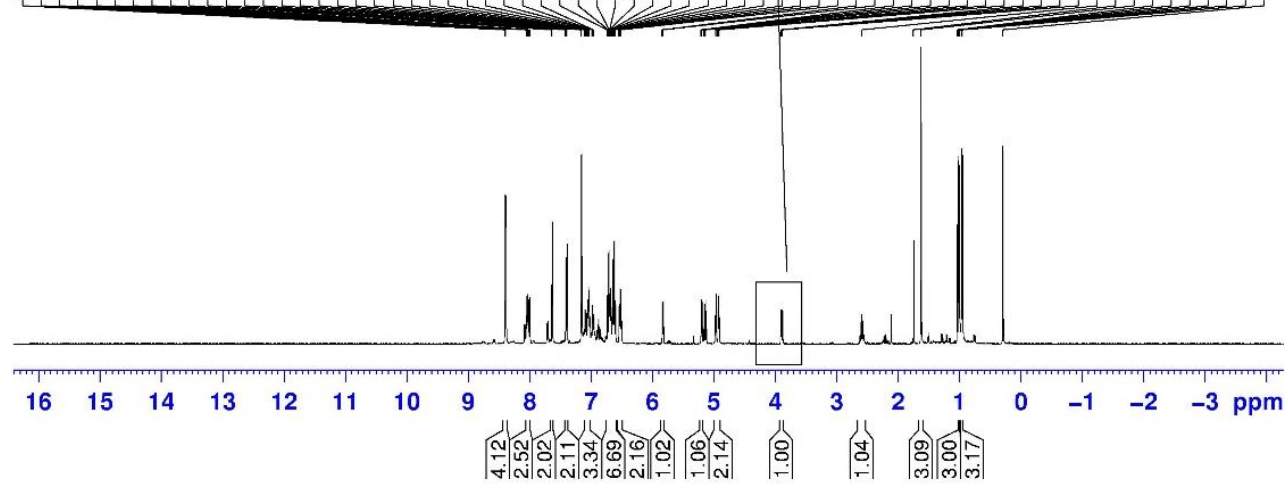

Current Data Parameters
NAME JUB881a 110102 EXPNO

F2 - Acquisition Parameters F2 - Acquisition Param
Date- 20160201
Time-
11.41

INSTRUM spect PULPROG $5 \mathrm{~mm}$ PABBO $\begin{array}{ll}\text { TD } & \\ \text { SOLVENT } & 99336 \\ \text { C6D6 }\end{array}$ NS $16^{\text {C6D }}$ SWH $\quad 8278.146 \mathrm{~Hz}$ FIDRES $\quad 0.083335 \mathrm{~Hz}$ $\begin{array}{lc}\text { AQ } & 5.9999442 \mathrm{sec} \\ \mathrm{RG} & 181 \\ \mathrm{DW} & 60.400 \mathrm{usec}\end{array}$ $\begin{array}{ll}\mathrm{DW} & 60.400 \mathrm{usec} \\ \mathrm{DE} & 6.00 \mathrm{usec}\end{array}$ $\begin{array}{ll}\text { TE } & 298.0 \mathrm{~K} \\ \mathrm{D} 1 & 1.00000000 \mathrm{sec}\end{array}$ $\begin{array}{lc}\text { TD0 } & 1.00000000 \\ \text { NUC1 } & 1 \\ \text { P } & 1 \mathrm{H}\end{array}$ $\begin{array}{ll}\text { NUC1 } & 1 \mathrm{H} \\ \text { P1 } & 10.30 \text { usec }\end{array}$ $\begin{array}{ll}\text { PLW1 } & -1.00000000 \mathrm{~W} \\ \text { SFO1 } & 400.1324710 \mathrm{MH}\end{array}$

2 - Processing parameters $\begin{array}{lr}\text { SI } & 131072 \\ \text { SF } & 400.1300444 \mathrm{MHz}\end{array}$ WSB SSB 00 $\begin{array}{lll}\mathrm{GB} & 0 & \\ \mathrm{PC} & & 1.40\end{array}$

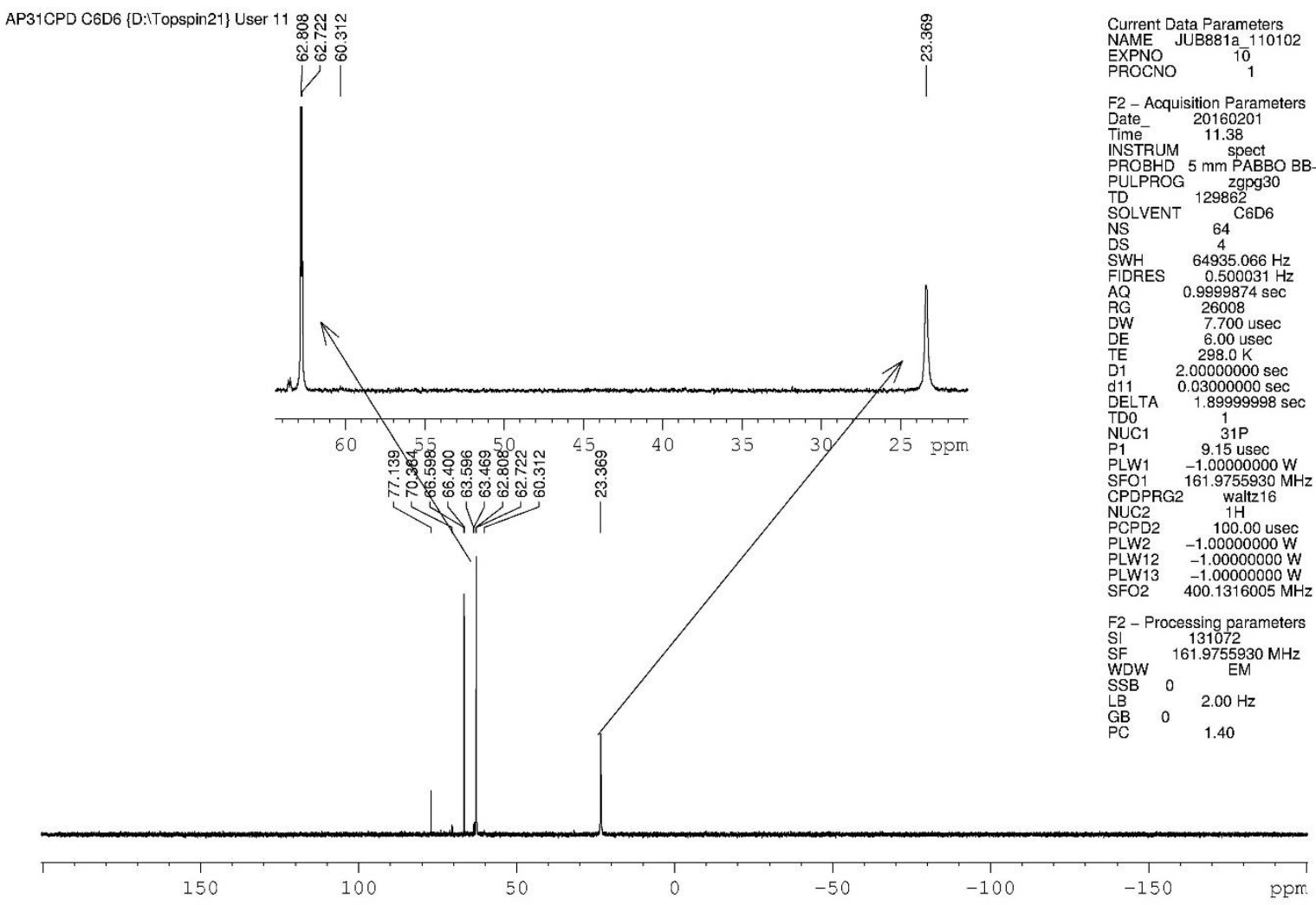

Figure S5. ${ }^{1} \mathrm{H}$ and ${ }^{31} \mathrm{P}\left\{{ }^{1} \mathrm{H}\right\}$ NMR spectra of $\mathbf{3 f}$ in $\mathrm{C}_{6} \mathrm{D}_{6}$. 
2. NMR spectra of the isolated complexes

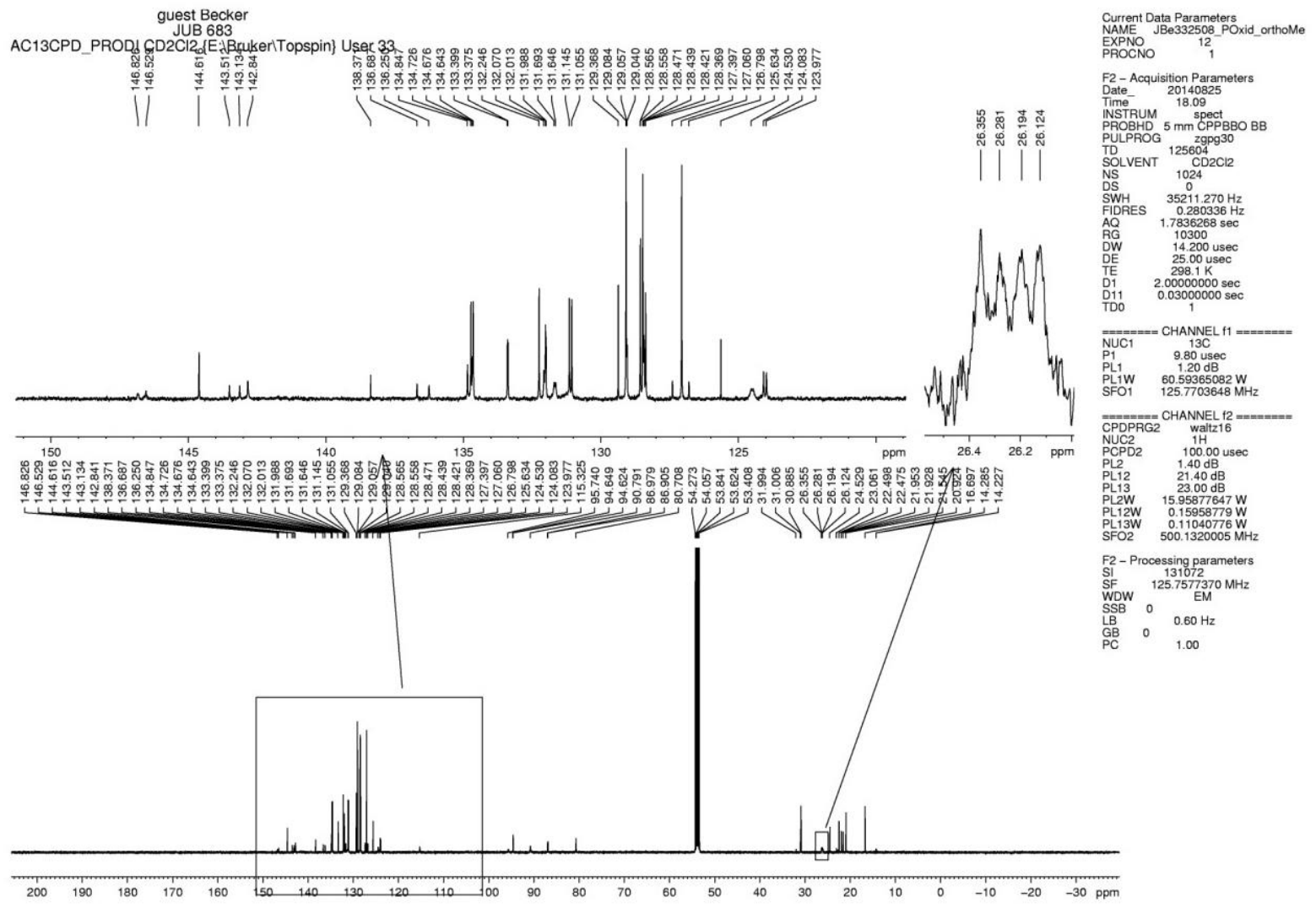

guest Becker
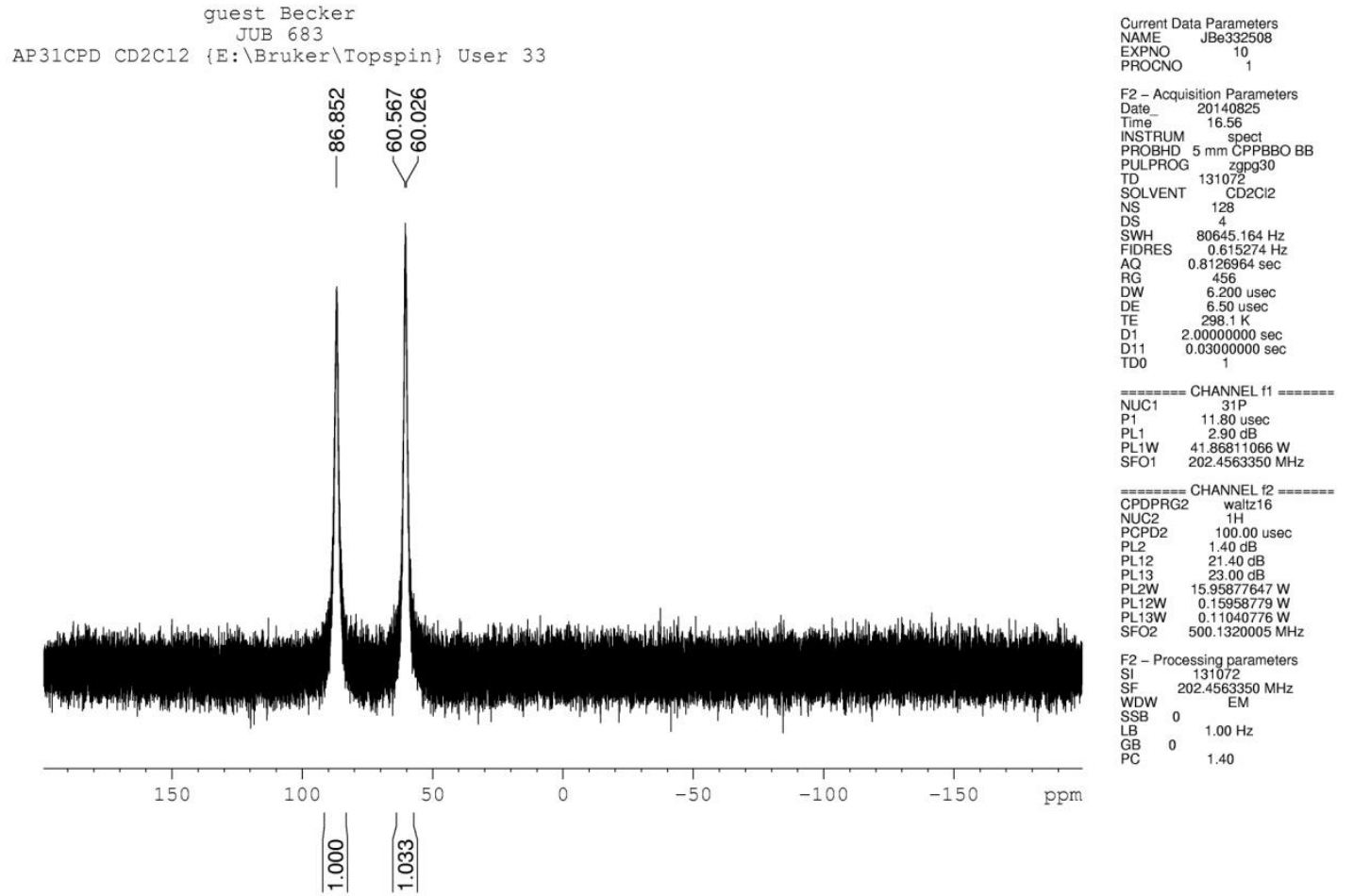

Figure S6. ${ }^{13} \mathrm{C}\left\{{ }^{1} \mathrm{H}\right\}$ and ${ }^{31} \mathrm{P}\left\{{ }^{1} \mathrm{H}\right\} \mathrm{NMR}$ spectra of $\mathbf{4 a}$ in $\mathrm{CD}_{2} \mathrm{Cl}_{2}$. 


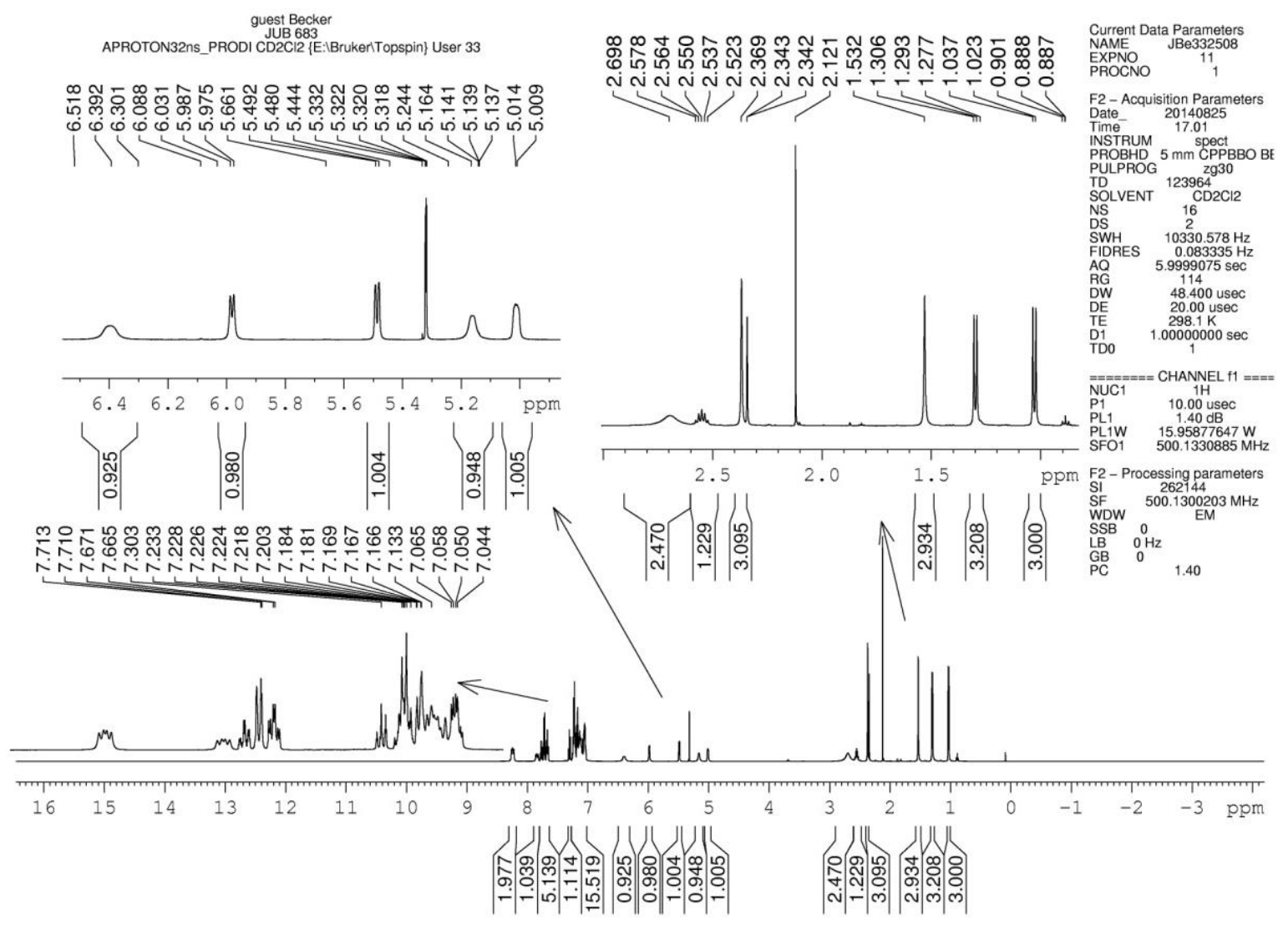

Figure S7. ${ }^{1} \mathrm{H}$ NMR spectrum of $4 \mathbf{a}$ in $\mathrm{CD}_{2} \mathrm{Cl}_{2}$.

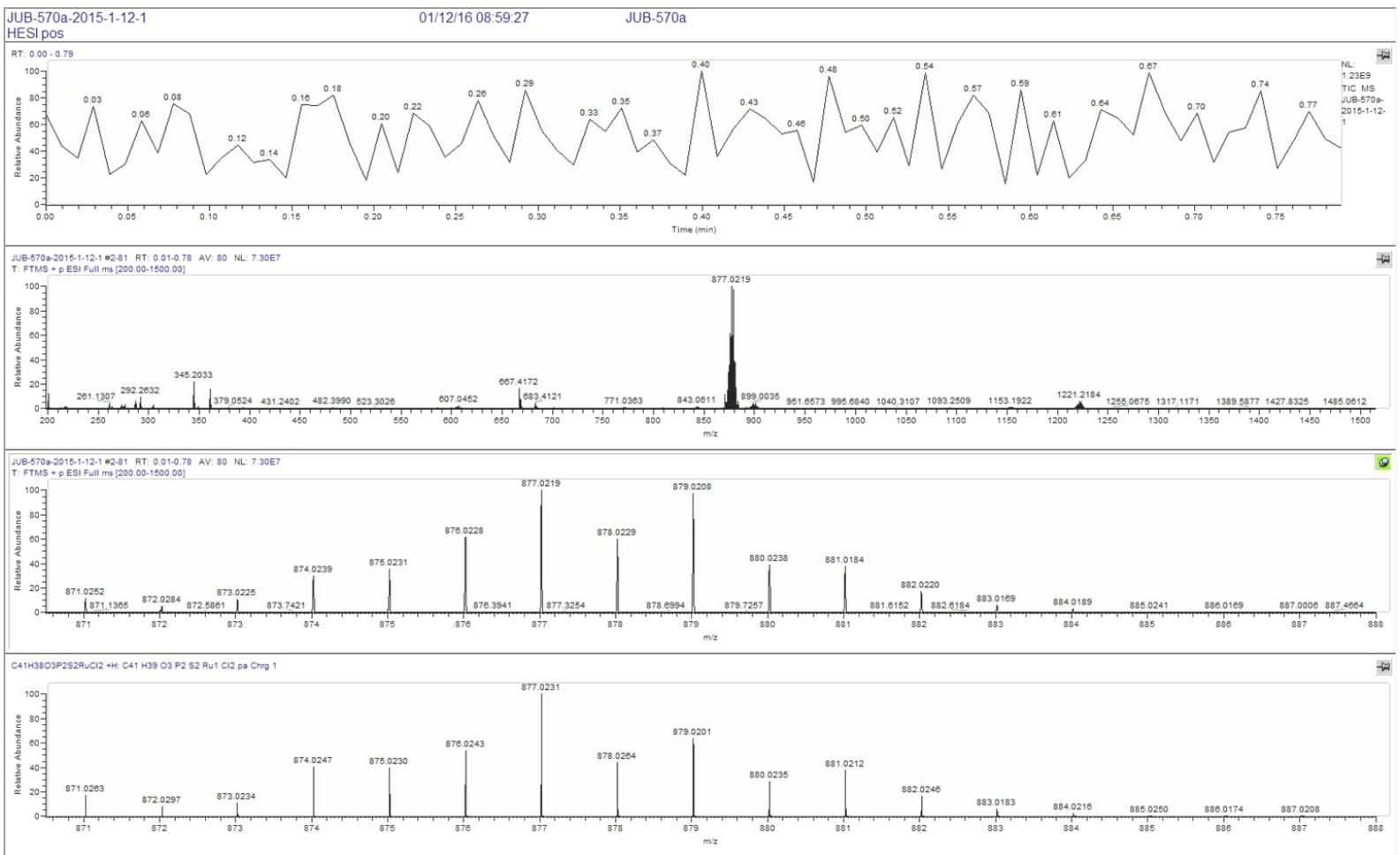

Figure S8. ESI-HR MS spectra of complex $\mathbf{4 b .}$ 

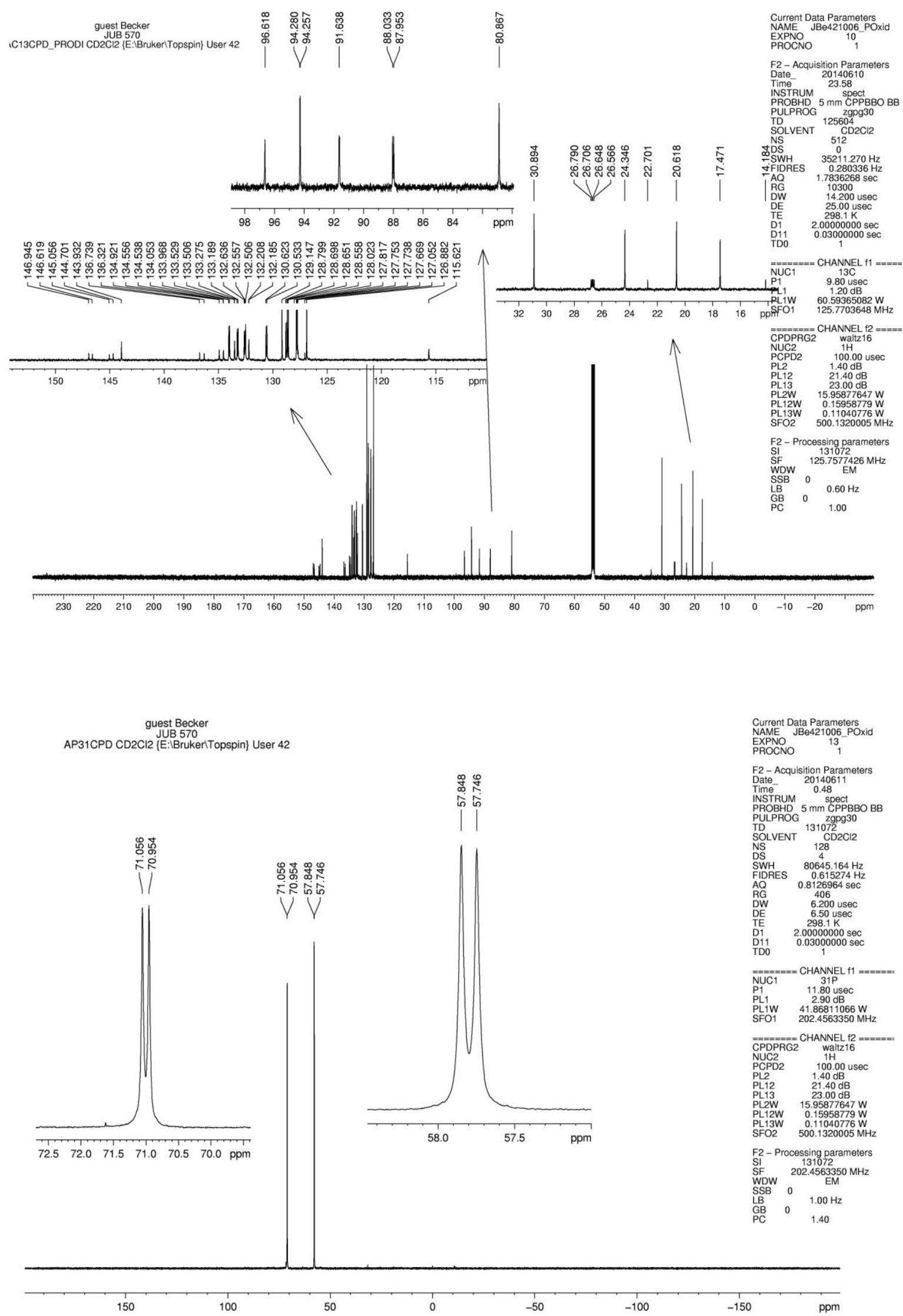

Figure S9. ${ }^{13} \mathrm{C}\left\{{ }^{1} \mathrm{H}\right\}$ and ${ }^{31} \mathrm{P}\left\{{ }^{1} \mathrm{H}\right\}$ NMR spectra of $\mathbf{4 b}$ in $\mathrm{CD}_{2} \mathrm{Cl}_{2}$. 


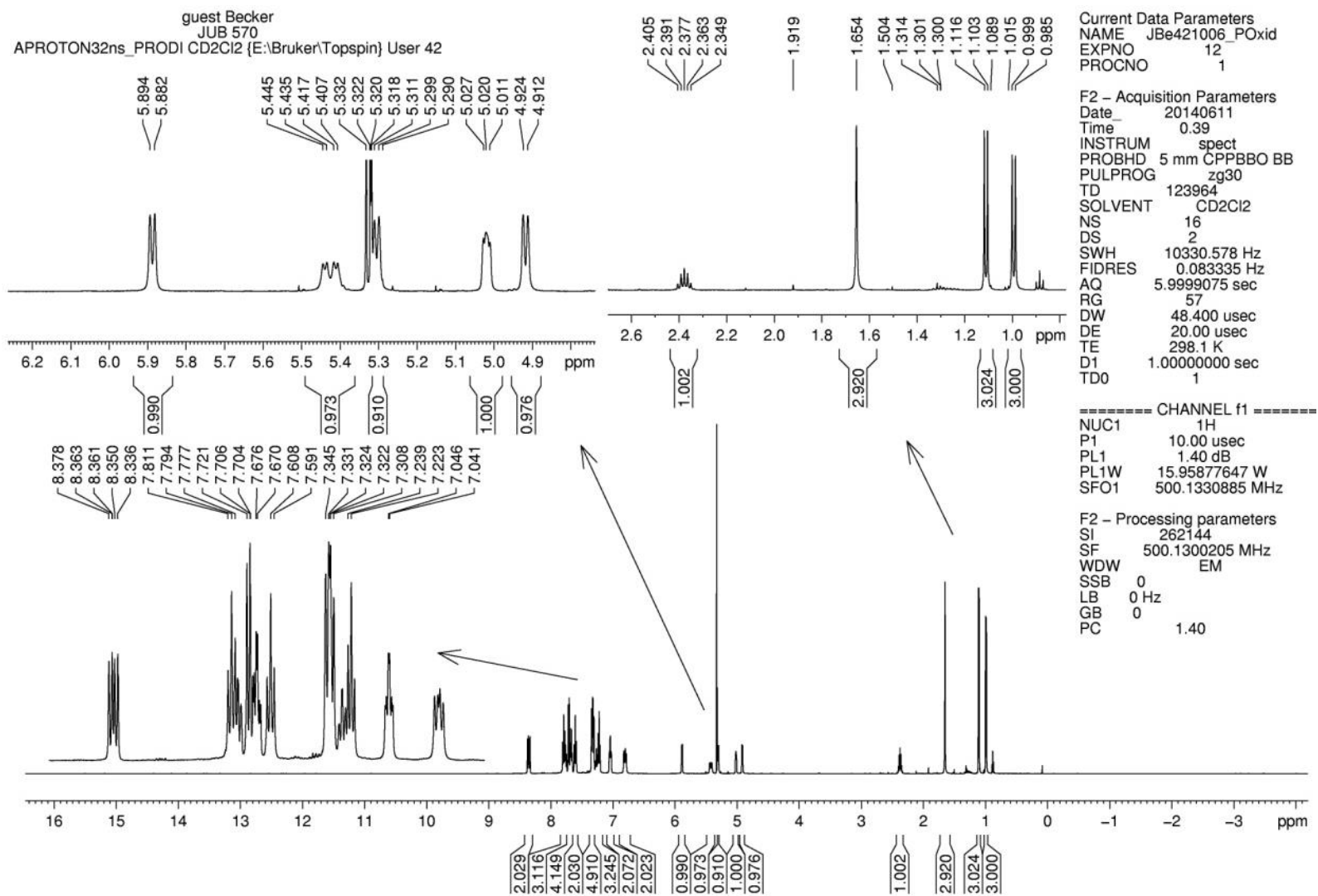

Figure S10. ${ }^{1} \mathrm{H}$ NMR spectrum of $\mathbf{4 b}$ in $\mathrm{CD}_{2} \mathrm{Cl}_{2}$.

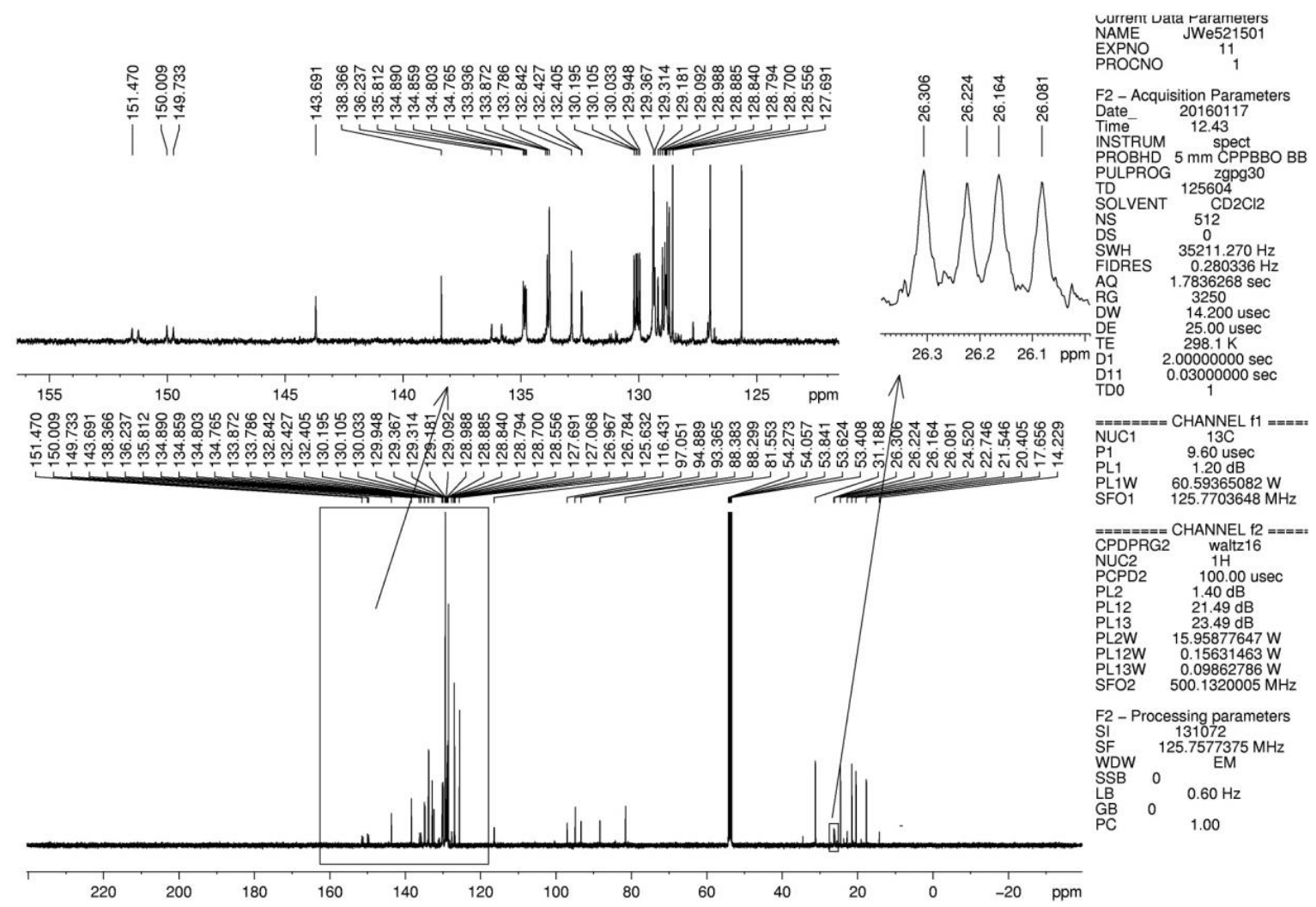

Figure S11. ${ }^{13} \mathrm{C}\left\{{ }^{1} \mathrm{H}\right\}$ NMR spectrum of $4 \mathbf{c}$ in $\mathrm{CD}_{2} \mathrm{Cl}_{2}$. 


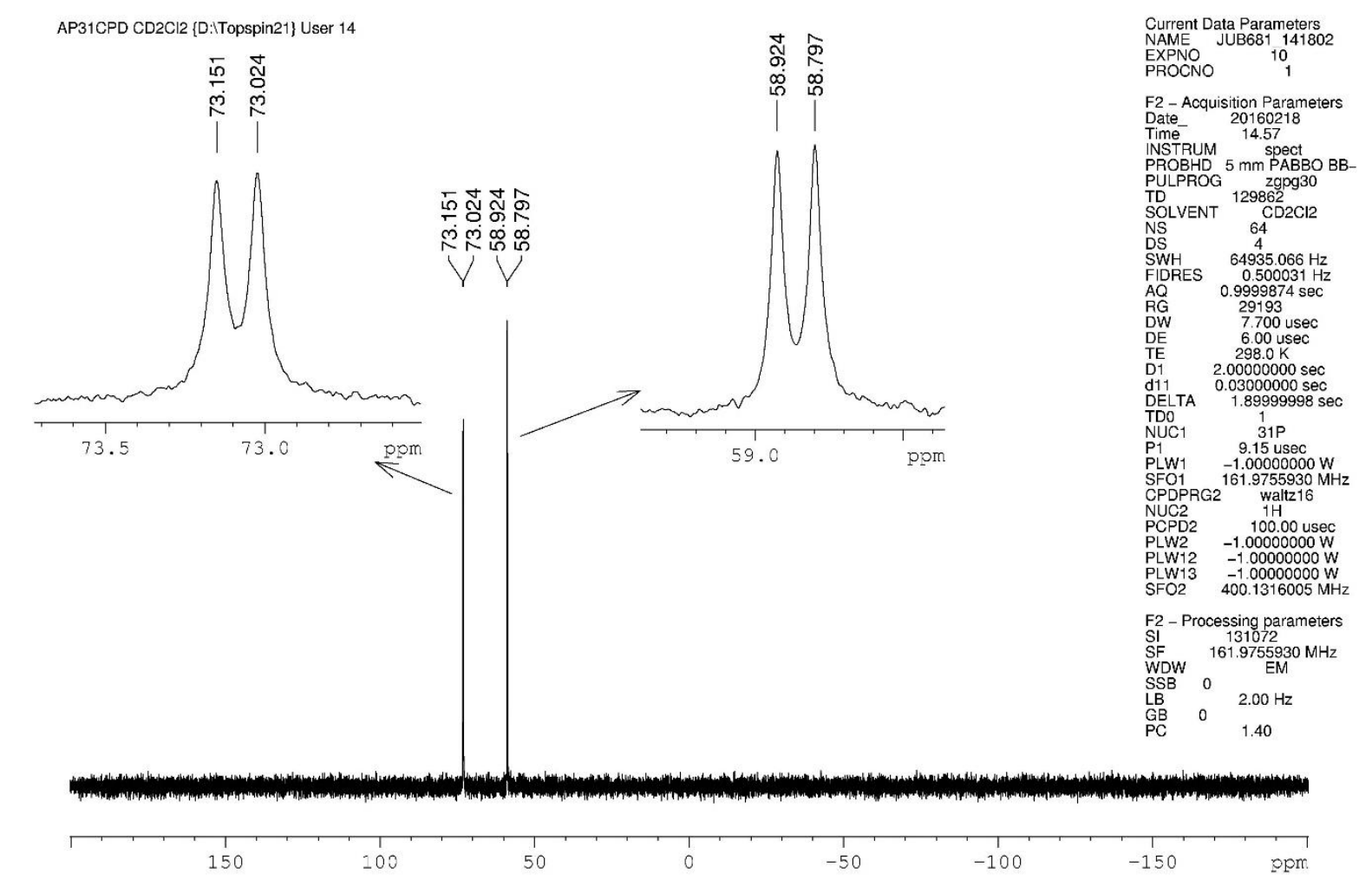

APROTON CD2Cl2 \{D:TTopspin21\} User 14

Current Data Parameters
NAME JUB681 141802
EXPNO
PROCNO

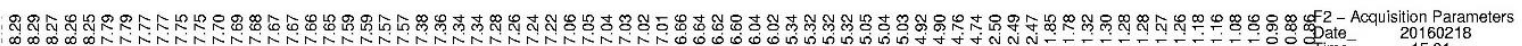

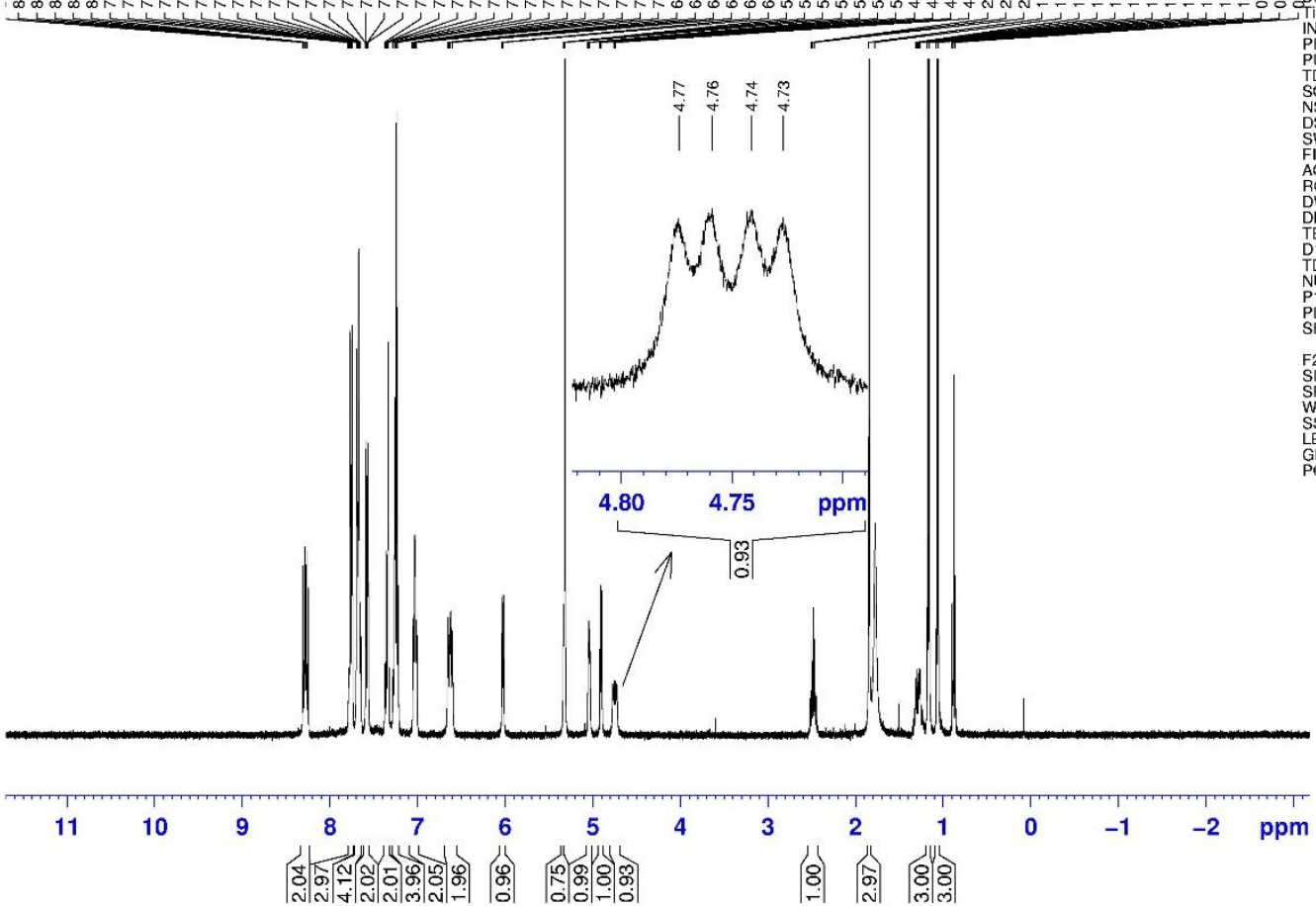

Figure S12. ${ }^{31} \mathrm{P}\left\{{ }^{1} \mathrm{H}\right\}$ and ${ }^{1} \mathrm{H}$ NMR spectra of $4 \mathbf{c}$ in $\mathrm{CD}_{2} \mathrm{Cl}_{2}$.

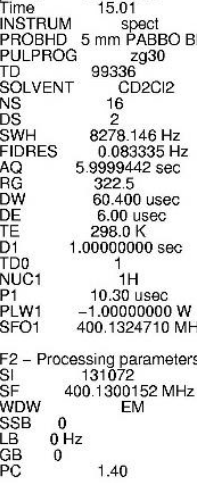

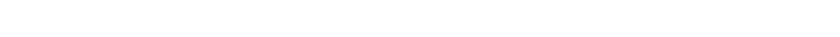




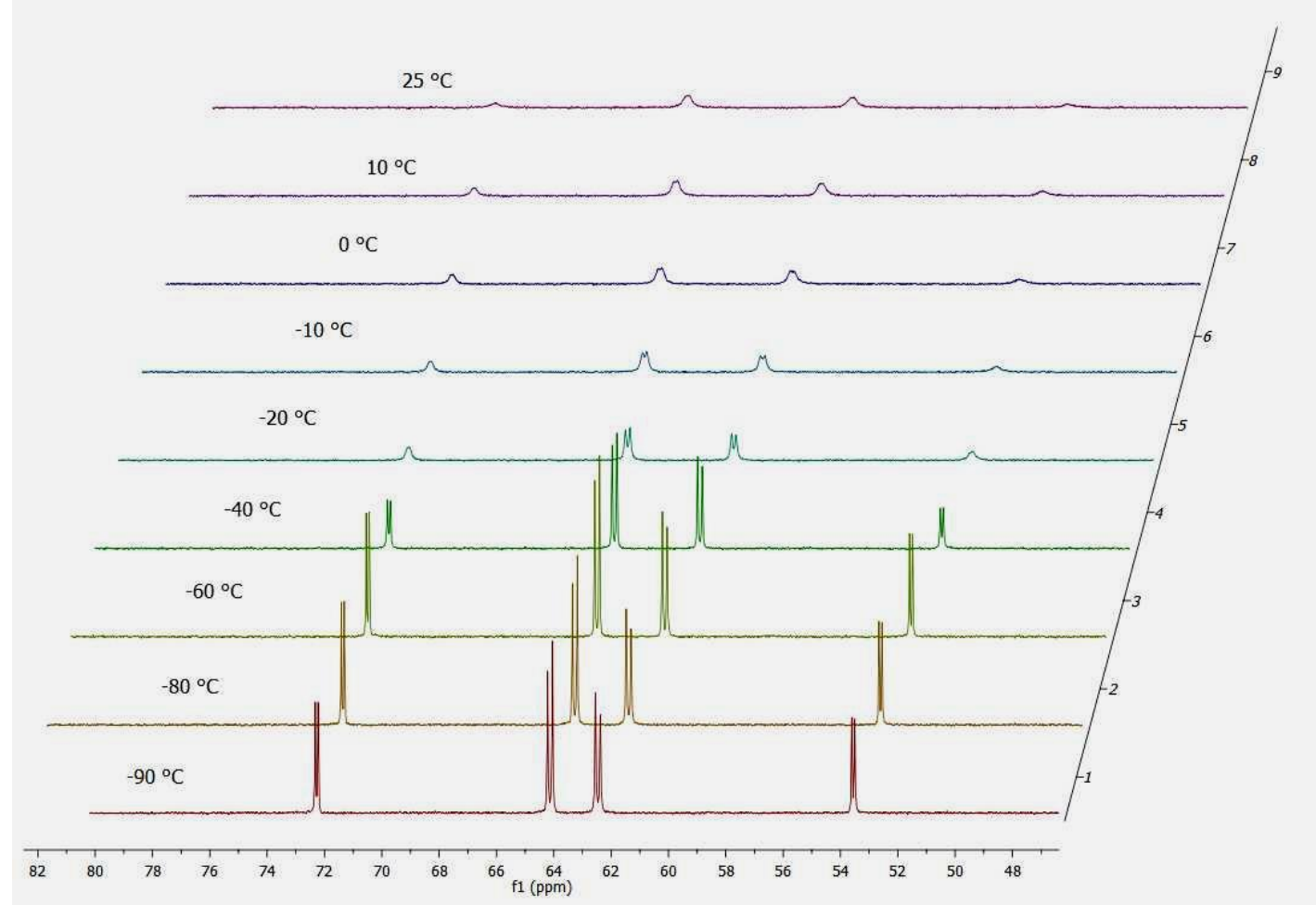

Figure S13a. VT ${ }^{31} \mathrm{P}\left\{{ }^{1} \mathrm{H}\right\} \mathrm{NMR}$ spectra of $\mathbf{5 a}$ in $\mathrm{CD}_{2} \mathrm{Cl}_{2}$ at low temperatures.

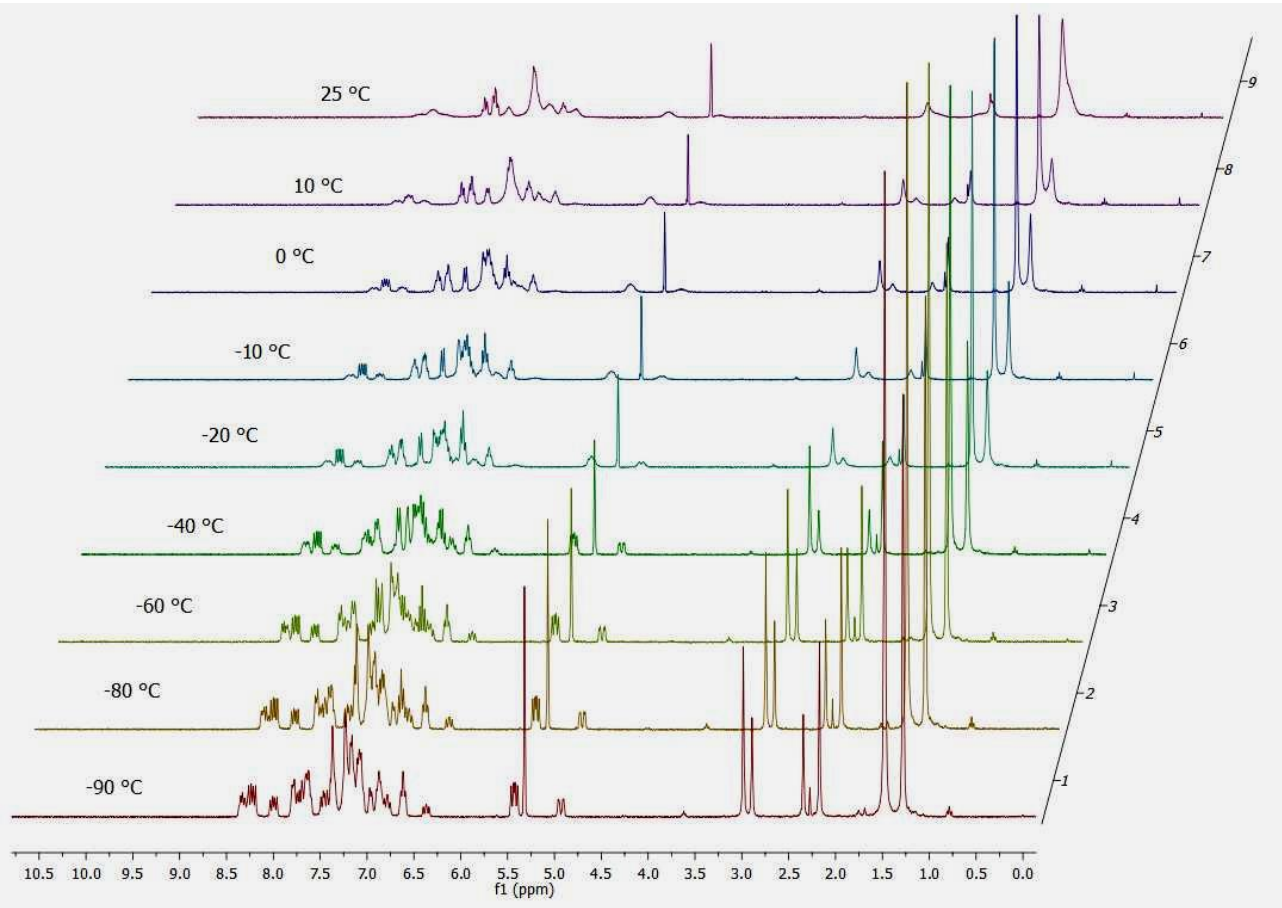

Figure S13b. VT ${ }^{1} \mathrm{H}$ NMR spectra of $\mathbf{5 a}$ in $\mathrm{CD}_{2} \mathrm{Cl}_{2}$ at low temperatures. 


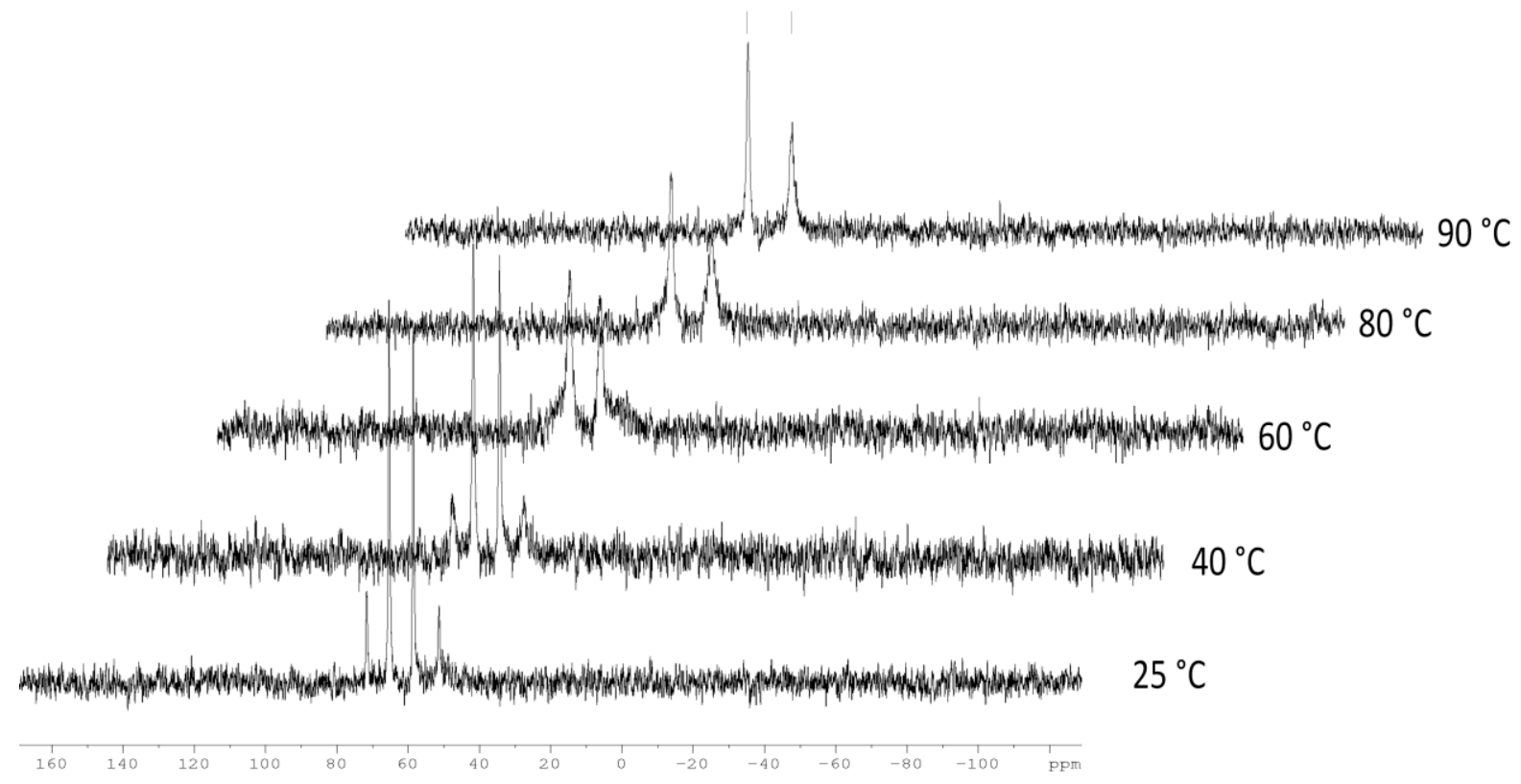

Figure 14a. VT ${ }^{31} \mathrm{P}\left\{{ }^{1} \mathrm{H}\right\}$ NMR spectra of $\mathbf{5 a}$ in $\mathrm{CD}_{2} \mathrm{Cl}_{2}$ at elevated temperatures. 
guest Weismann
JUB POMe

Messung bei $-90^{\circ} \mathrm{C}$
AC13CPD CD2Cl2 $\{$ E:IBrukerlTopspin\} User 40

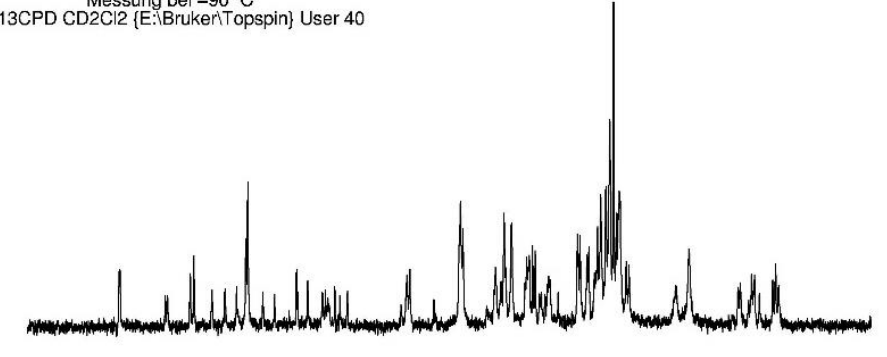

$\begin{array}{llllllllllllll}146 & 144 & 142 & 140 & 138 & 136 & 134 & 132 & 130 & 128 & 126 & 124 & 122 & \text { ppm }\end{array}$
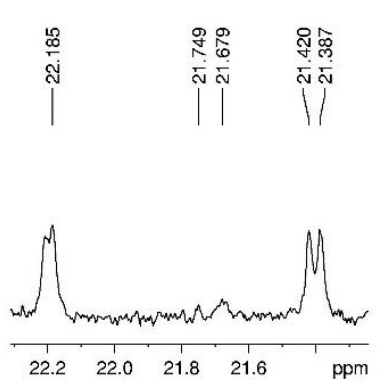

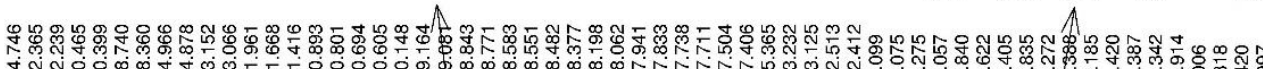

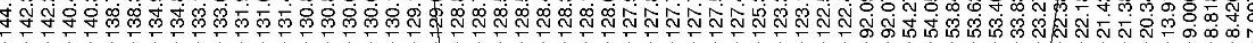

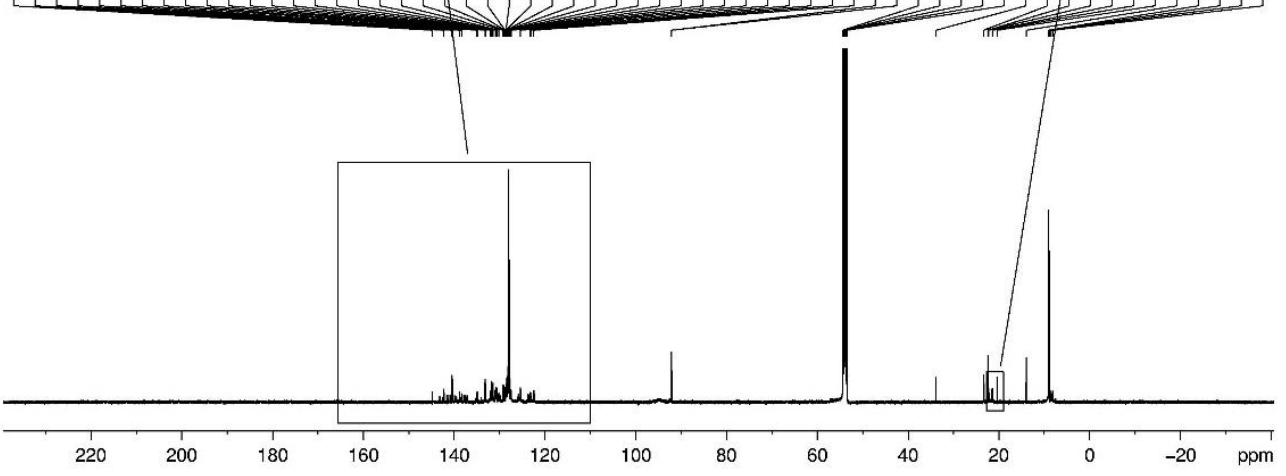

Current Data Parameters

EXPNO 14

PROCNO

F2 - Acquisition Parameters

$\begin{array}{ccc}2016011 \\ \text { Time } & 10.24\end{array}$

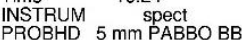

PULPROG zgpg30

SOLVENT ${ }^{125782} \mathrm{CD} 2 \mathrm{Cl} 2$

NS 4096

$\begin{array}{ll}4 & 4 \\ \text { WH } & 35211.270 \mathrm{~Hz}\end{array}$

IDRES $\quad 0.279939 \mathrm{H}$

$\begin{array}{ll}A Q & 1.7861544 \mathrm{sec} \\ \mathrm{RG} & 4600\end{array}$

14.200 usec

6.50 usec

$2.00000000 \mathrm{sec}$
$0.03000000 \mathrm{sec}$

$\begin{array}{ll}\text { D11 } & 0.03000000 \mathrm{sec} \\ \text { TD0 } & 1\end{array}$

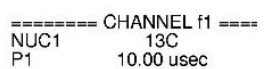

$\quad 10.00$ usec

$\begin{array}{ll}\text { PL1W } & 43.89622116 \mathrm{~W} \\ \text { SFO1 } & 125.7703648 \mathrm{MHz}\end{array}$

$======$
CPDPRG2 CHANNEL $f 2===$ $1 \mathrm{H}$
$\mathrm{NUC2}$

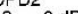

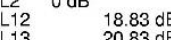

PL2W $22.02924347 \mathrm{~W}$

PLI2W $0.28840289 \mathrm{~W}$

$\begin{array}{ll}\text { SL13W } & 0.18196993 \mathrm{~W} \\ \text { SFO2 } & 500.1320005 \mathrm{MH}\end{array}$

2 - Processing parameters

$\begin{array}{ll}\text { SI } & 131072 \\ \text { SF } & 125.7578669 \mathrm{MHz}\end{array}$

WDW EM

$\begin{array}{lll}\mathrm{LB} & 0 & 0.60 \mathrm{~Hz} \\ \mathrm{~GB} & 0 & 1.00\end{array}$

Figure S15. ${ }^{13} \mathrm{C}\left\{{ }^{1} \mathrm{H}\right\}$ NMR spectra of 5a in $\mathrm{CD}_{2} \mathrm{Cl}_{2}$.

guest Becke

AC13CPD PRODI CD2CI2 737 :B:BrukerlTopspin\} User 39

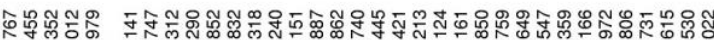

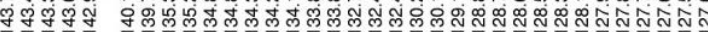

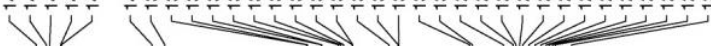
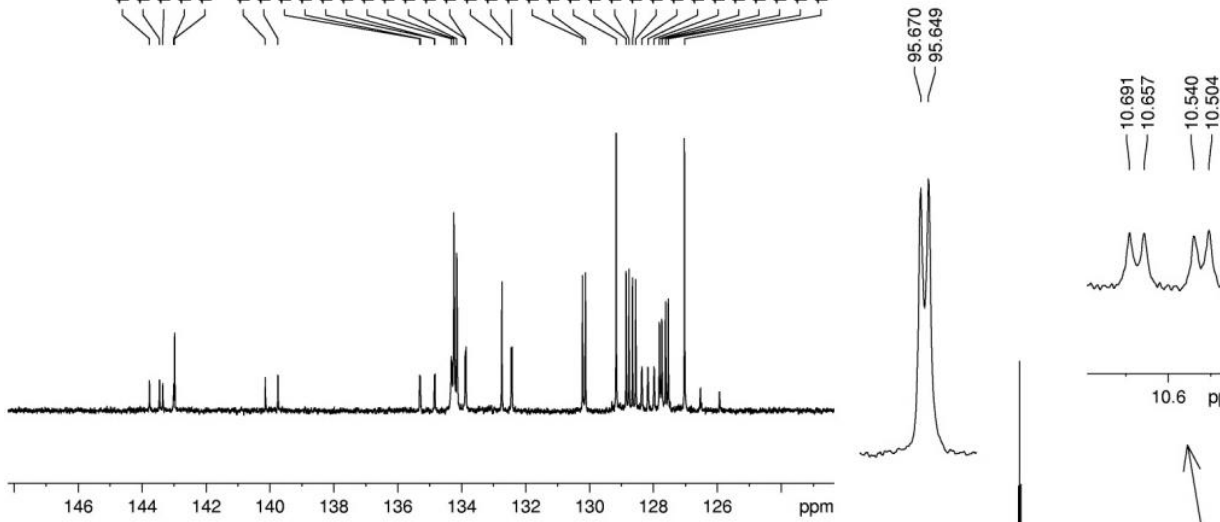

Current Data Parameters
NAME JBe390710

NAME ROCNO

F2 - Acquisition Parameters

20141007
Time

INSTRUM spect PROBHD $5 \mathrm{~mm}$ CPPBB

TD ${ }_{125604}^{2 g p g} 30$

SOLVENT 12560402

NS

$\begin{array}{lc}\text { DS } & 512 \\ \text { SWH } & 35211.270 \mathrm{~Hz}\end{array}$

$\begin{array}{lr}\text { SWH } & 35211.270 \mathrm{~Hz} \\ \text { FIDRES } & 0.280336 \mathrm{~Hz}\end{array}$

$\begin{array}{lc}\text { AQ } & 1.7836268 \mathrm{sec} \\ \text { RG } & 10300\end{array}$

$\begin{array}{ll}\text { RG } & 10300 \\ \text { DW } & 14.200 \text { usec }\end{array}$

$\begin{array}{ll}\text { DE } & 25.00 \text { usec } \\ \text { TE } & 298.1 \mathrm{~K}\end{array}$

$\begin{array}{ll}\text { D1 } & 2.00000000 \mathrm{sec} \\ \text { D11 } & 0.03000000 \mathrm{sec}\end{array}$

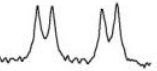

$======$ CHANNEL $f 1=$
NUC1 $13 \mathrm{C}=$
P1 $\quad 9.80 \mathrm{usec}$

$\begin{array}{ll}\text { PL1W } & 60.59365082 \mathrm{~W} \\ \text { SFO1 } & 125.7703648 \mathrm{MH}\end{array}$

$10.6 \mathrm{ppm}$

$===== \pm$ CHANNEL $12=$
CPDPRG2

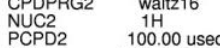

$\begin{array}{ll}100.00 \\ \mathrm{PL2} & 1.40 \mathrm{~dB}\end{array}$

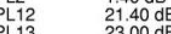

PL2W 15.95877647 W

$\begin{array}{ll}\text { PL12W } & 0.1595879 \mathrm{~W} \\ \mathrm{PL} & \mathrm{W}\end{array}$

SFO2 $500.1320005 \mathrm{MH}$

F2 - Processing parameters

131072

SFW $125.7577376 \mathrm{MHz}$

SSB

LB $0.60 \mathrm{~Hz}$

100

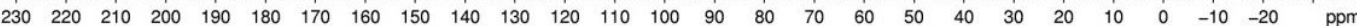

Figure S16. ${ }^{13} \mathrm{C}\left\{{ }^{1} \mathrm{H}\right\}$ spectrum of $\mathbf{5 b}$ in $\mathrm{CD}_{2} \mathrm{Cl}_{2}$. 


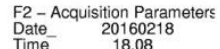

Time 18.08

$\begin{array}{ll}\text { INSTRUM } & \text { S. } \\ \text { PRect } \\ \text { PROBDO }\end{array}$

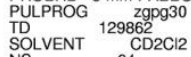

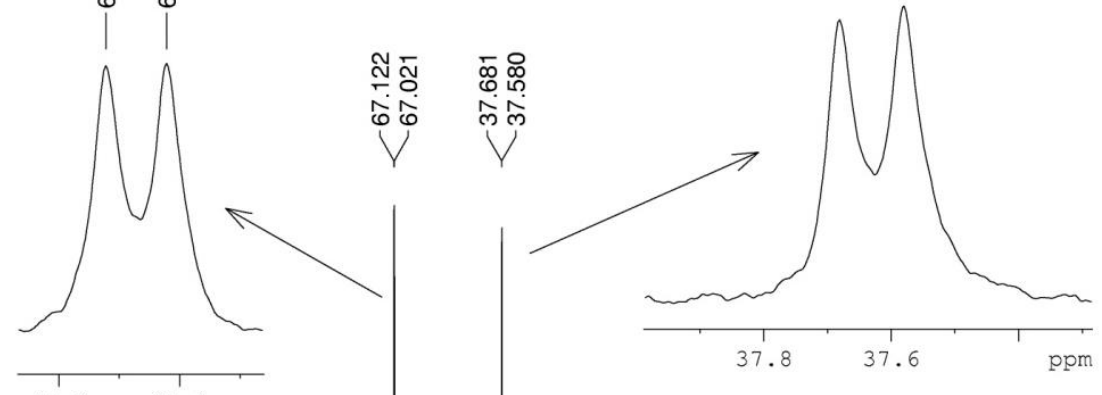

$\begin{array}{lll}\text { SOLVENT } & \text { CD2 } \\ \text { NS } & 64\end{array}$

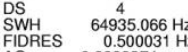

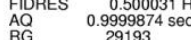

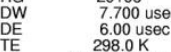

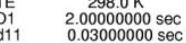

DELTA $0.030 .09999998 \mathrm{sec}$

NU0 11 1 31 P

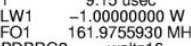

WC2

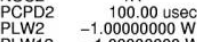

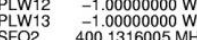

F2 - Processing parameters

SI $161.9755930 \mathrm{MHz}$

WDW 0 EM

$\begin{array}{lll} & \\ \text { GB } & 0 & 2.00 \mathrm{H} \\ \mathrm{GC} & & 1.40\end{array}$

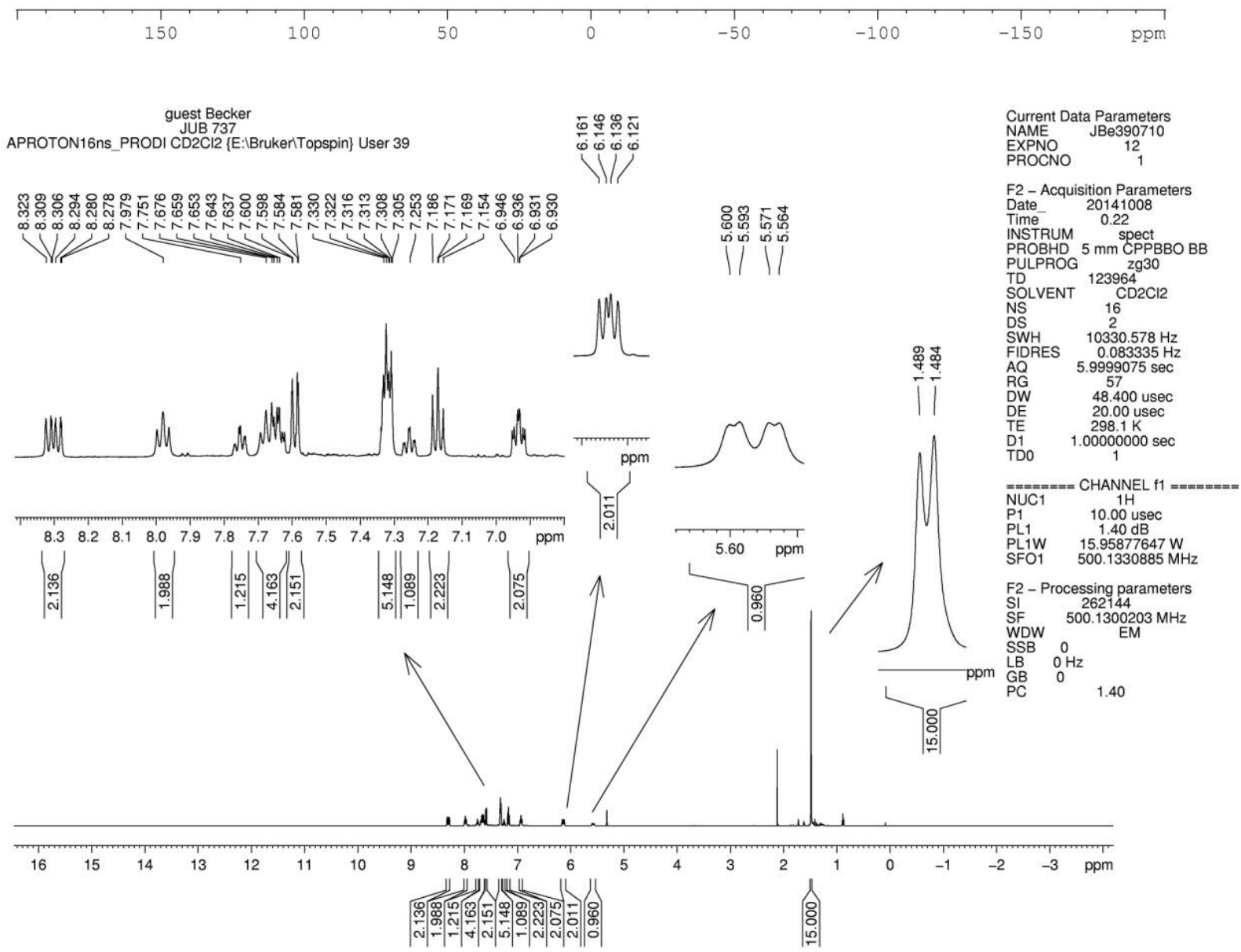

Figure S17. ${ }^{1} \mathrm{H}$ and ${ }^{31} \mathrm{P}\left\{{ }^{1} \mathrm{H}\right\}$ NMR spectra of $\mathbf{5 b}$ in $\mathrm{CD}_{2} \mathrm{Cl}_{2}$. 


\section{Crystal Structure Determination}

Table S1a. Data collection and structure refinement details for compounds $\mathbf{4 a}$ and $\mathbf{4 b}$.

\begin{tabular}{|c|c|c|}
\hline Compound & $4 \mathbf{a}$ & $4 b$ \\
\hline CCDC No. & CCDC 1450798 & CCDC 1450797 \\
\hline Formula & $\mathrm{C}_{43} \mathrm{H}_{43} \mathrm{O}_{3} \mathrm{P}_{2} \mathrm{RuS}_{2} \cdot 0.5 \mathrm{C}_{7} \mathrm{H}_{8}$ & $\mathrm{C}_{44} \mathrm{H}_{41} \mathrm{O}_{3} \mathrm{P}_{2} \mathrm{RuS}_{2} \mathrm{Cl}_{2}$ \\
\hline $\begin{array}{l}\text { Formula weight } \\
{\left[\mathrm{g} \cdot \mathrm{mol}^{-1}\right]}\end{array}$ & 880.97 & 915.80 \\
\hline Temperature [K] & $100(2)$ & $100(2)$ \\
\hline Wave length [̊] & 0.71073 & 0.71073 \\
\hline Crystal system & Monoclinic & Monoclinic \\
\hline Space group & $C 2 / \mathrm{c}$ & $C 2 / \mathrm{c}$ \\
\hline $\mathrm{a}[\AA]$ & $25.0763(13)$ & $27.165(3)$ \\
\hline $\mathrm{b}[\AA]$ & $12.6879(7)$ & $13.074(1)$ \\
\hline$c[\AA]$ & $26.8084(14)$ & $25.394(4)$ \\
\hline$\alpha\left[^{\circ}\right]$ & 90 & 90 \\
\hline$\beta\left[^{\circ}\right]$ & $109.414(2)$ & $118.508(3)$ \\
\hline$\gamma\left[{ }^{\circ}\right]$ & 90 & 90 \\
\hline Volume $\left[\AA^{3}\right]$ & $8044.5(7)$ & $7925.2(17)$ \\
\hline $\mathrm{Z}$ & 8 & 8 \\
\hline Calc. density $\left[\mathrm{Mg} \cdot \mathrm{m}^{-3}\right]$ & 1.455 & 1.535 \\
\hline$\mu\left(\mathrm{Mo}_{\mathrm{K} \alpha}\right)\left[\mathrm{mm}^{-1}\right]$ & 0.615 & 0.758 \\
\hline $\mathrm{F}(000)$ & 3648 & 3752 \\
\hline Crystal dimensions [mm] & $0.21 \times 0.19 \times 0.09$ & $0.19 \times 0.18 \times 0.07$ \\
\hline Theta range $\left[{ }^{\circ}\right]$ & 1.61 to 25.00 & 2.90 to 25 \\
\hline \multirow[t]{3}{*}{ Index ranges } & $-29 \leq \mathrm{h} \leq 29$ & $-32 \leq \mathrm{h} \leq 32$ \\
\hline & $-15 \leq \mathrm{k} \leq 15$ & $-15 \leq \mathrm{k} \leq 15$ \\
\hline & $-31 \leq 1 \leq 31$ & $-30 \leq 1 \leq 30$ \\
\hline Reflections collected & 59370 & 44370 \\
\hline Independent reflections & $7089\left[R_{\mathrm{int}}=0.0496\right]$ & $6950\left[R_{\mathrm{int}}=0.0969\right]$ \\
\hline Data/Restraints/Parameter & $7089 / 0 / 517$ & $6950 / 0 / 494$ \\
\hline Goodness-of-fit on $\mathrm{F}^{2}$ & 1.097 & 1.100 \\
\hline Final $R$ indices $[\mathrm{I}>2 \operatorname{sigma}(\mathrm{I})]$ & $\begin{array}{l}R 1=0.0413 \\
\mathrm{w} R 2=0.1229\end{array}$ & $\begin{array}{l}R 1=0.0381 \\
\mathrm{w} R 2=0.0661\end{array}$ \\
\hline$R$ indices (all data) & $\begin{array}{l}R 1=0.0486 \\
\mathrm{w} R 2=0.1275\end{array}$ & $\begin{array}{l}R 1=0.0738 \\
\mathrm{w} R 2=0.0774\end{array}$ \\
\hline Largest diff. peak and hole & 0779 und -0.793 & 0.417 und -0.428 \\
\hline
\end{tabular}

Table S1b. Data collection and structure refinement details for compounds $\mathbf{5 a}$ and $\mathbf{5 b}$.

\begin{tabular}{lll}
\hline Compound & $\mathbf{5 a}$ & $\mathbf{5 b}$ \\
\hline \hline CCDC No. & $\mathrm{CCDC} 1450796$ & $\mathrm{CCDC} \mathrm{1450795}$ \\
Formula & $\mathrm{C}_{43} \mathrm{H}_{45} \mathrm{O}_{3} \mathrm{P}_{2} \mathrm{IrS}_{2}$ & $\mathrm{C}_{41} \mathrm{H}_{39} \mathrm{Cl}_{2} \mathrm{IrO}_{3} \mathrm{P}_{2} \mathrm{~S}_{2} \cdot 1.5 \mathrm{C}_{6} \mathrm{H}_{6}$ \\
Formula weight $\left[\mathrm{g} \cdot \mathrm{mol}^{-1}\right]$ & 928.05 & 1086.05 \\
Temperature $[\mathrm{K}]$ & $100(2)$ & $100(2)$ \\
Wave length $[\AA]$ & 0.71073 & 0.71073 \\
Crystal system & Monoclinic & Monoclinic \\
Space group & $P 2{ }_{1} / \mathrm{c}$ & $P 2{ }_{1} / \mathrm{n}$ \\
a $[\AA]$ & $11.5779(12)$ & $13.0190(6)$ \\
b $[\AA]$ & $19.1195(19)$ & $23.4383(10)$
\end{tabular}




\begin{tabular}{|c|c|c|}
\hline$c[\AA]$ & $17.1015(17)$ & 18. $9496(7)$ \\
\hline$\alpha\left[^{\circ}\right]$ & 90 & 90 \\
\hline$\beta\left[{ }^{\circ}\right]$ & $96.535(3)$ & $95.295(2)^{\circ}$ \\
\hline$\gamma\left[{ }^{\circ}\right]$ & 90 & 90 \\
\hline Volume $\left[\AA^{3}\right]$ & $3761.1(7)$ & $4542.3(4)$ \\
\hline $\mathrm{Z}$ & 4 & 4 \\
\hline Calc. density $\left[\mathrm{Mg} \cdot \mathrm{m}^{-3}\right]$ & 1.639 & 1.588 \\
\hline$\mu\left(\mathrm{Mo}_{\mathrm{K} \alpha}\right)\left[\mathrm{mm}^{-1}\right]$ & 3.787 & 3.262 \\
\hline $\mathrm{F}(000)$ & 1864 & 2180 \\
\hline Crystal dimensions [mm] & $0.31 \times 0.28 \times 0.21$ & $0.21 \times 0.20 \times 0.11$ \\
\hline Theta range $\left[{ }^{\circ}\right]$ & 1.60 to 26.44 & 1.62 to 25.00 \\
\hline \multirow[t]{3}{*}{ Index ranges } & $-13 \leq \mathrm{h} \leq 13$ & $-15 \leq \mathrm{h} \leq 15$ \\
\hline & $-22 \leq \mathrm{k} \leq 22$ & $-27 \leq \mathrm{k} \leq 27$ \\
\hline & $-20 \leq 1 \leq 20$ & $-17 \leq 1 \leq 17$ \\
\hline Reflections collected & 45901 & 74061 \\
\hline Independent reflections & $6633\left[R_{\mathrm{int}}=0.0426\right]$ & $8005\left[R_{\mathrm{int}}=0.0352\right]$ \\
\hline Data/Restraints/Parameter & $6633 / 0 / 461$ & $8005 / 0 / 550$ \\
\hline Goodness-of-fit on $\mathrm{F}^{2}$ & 1.044 & 1.013 \\
\hline Final $R$ indices $[I>2 \operatorname{sigma}(I)]$ & $\begin{array}{l}R 1=0.0198 \\
\mathrm{w} R 2=0.0425\end{array}$ & $\begin{array}{l}R 1=0.0201 \\
\mathrm{w} R 2=0.0468\end{array}$ \\
\hline$R$ indices (all data) & $\begin{array}{l}R 1=0.0255 \\
\mathrm{w} R 2=0.0445\end{array}$ & $\begin{array}{l}R 1=0.0249 \\
\mathrm{w} R 2=0.0493\end{array}$ \\
\hline Largest diff. peak and hole & 0.467 and -0.397 & 0.797 and -0.366 \\
\hline
\end{tabular}

\subsection{Crystal Structure Determination of $\mathbf{4 a}$}

All hydrogen atoms were refined on ideal positions except for $\mathrm{H} 1$ at $\mathrm{C} 1$, which was found in the difference Fourier map and refined independently.

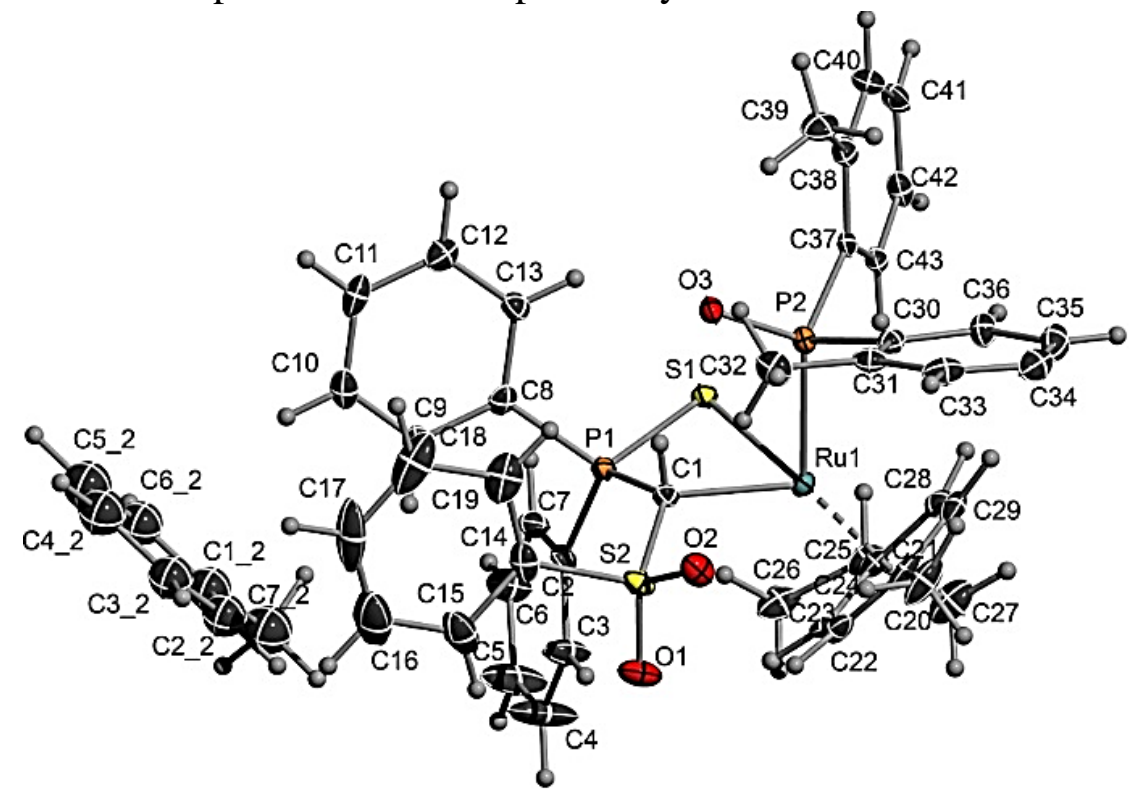

Figure S18. ORTEP Plot of complex 4a. Ellipsoids are drawn at the 50\% probability level. 
Table S2. Atomic coordinates $\left(\mathrm{x} 10^{4}\right)$ and equivalent isotropic displacement parameters $\left(\AA^{2} \times 10^{3}\right)$ for $4 \mathbf{a}$. $\mathrm{U}(\mathrm{eq})$ is defined as one third of the trace of the orthogonalized $\mathrm{U}^{\mathrm{ij}}$ tensor.

\begin{tabular}{|c|c|c|c|c|}
\hline & $\mathrm{X}$ & $\mathrm{y}$ & $\mathrm{Z}$ & $\mathrm{U}(\mathrm{eq})$ \\
\hline $\mathrm{Ru}(1)$ & $8207(1)$ & $10758(1)$ & $8434(1)$ & $12(1)$ \\
\hline $\mathrm{S}(1)$ & $8063(1)$ & $9027(1)$ & 7999(1) & $13(1)$ \\
\hline $\mathrm{S}(2)$ & $8819(1)$ & $9992(1)$ & $9721(1)$ & $16(1)$ \\
\hline$P(1)$ & $8545(1)$ & 8536(1) & $8723(1)$ & $12(1)$ \\
\hline$P(2)$ & $7262(1)$ & $10441(1)$ & $8417(1)$ & $12(1)$ \\
\hline $\mathrm{O}(1)$ & $9393(1)$ & $10233(2)$ & $9767(1)$ & $24(1)$ \\
\hline $\mathrm{O}(2)$ & $8509(1)$ & $10800(2)$ & $9885(1)$ & $21(1)$ \\
\hline $\mathrm{O}(3)$ & 7194(1) & $9515(2)$ & $8748(1)$ & $15(1)$ \\
\hline $\mathrm{C}(1)$ & $8412(2)$ & $9630(3)$ & $9079(1)$ & $13(1)$ \\
\hline $\mathrm{C}(2)$ & $9245(2)$ & $8241(3)$ & $8716(1)$ & $16(1)$ \\
\hline $\mathrm{C}(3)$ & $9742(2)$ & $8722(3)$ & $9020(2)$ & $30(1)$ \\
\hline $\mathrm{C}(4)$ & $10242(2)$ & $8484(5)$ & $8928(2)$ & $56(2)$ \\
\hline $\mathrm{C}(5)$ & $10247(2)$ & $7766(4)$ & $8540(2)$ & $52(2)$ \\
\hline $\mathrm{C}(6)$ & $9753(2)$ & $7275(3)$ & $8248(2)$ & $30(1)$ \\
\hline$C(7)$ & $9252(2)$ & $7503(3)$ & $8334(2)$ & $20(1)$ \\
\hline $\mathrm{C}(8)$ & $8288(2)$ & $7335(3)$ & $8927(1)$ & $16(1)$ \\
\hline $\mathrm{C}(9)$ & $8649(2)$ & $6687(3)$ & $9306(1)$ & $19(1)$ \\
\hline$C(10)$ & $8431(2)$ & $5849(3)$ & $9507(2)$ & $24(1)$ \\
\hline$C(11)$ & $7856(2)$ & $5644(3)$ & $9318(2)$ & $24(1)$ \\
\hline $\mathrm{C}(12)$ & $7499(2)$ & $6283(3)$ & $8935(2)$ & $22(1)$ \\
\hline$C(13)$ & $7708(2)$ & $7142(3)$ & $8744(2)$ & $18(1)$ \\
\hline $\mathrm{C}(14)$ & $8837(2)$ & $8885(3)$ & 10127(1) & $22(1)$ \\
\hline$C(15)$ & $8335(2)$ & $8469(3)$ & $10142(2)$ & $34(1)$ \\
\hline$C(16)$ & $8361(3)$ & $7619(4)$ & $10483(2)$ & $48(1)$ \\
\hline$C(17)$ & $8885(3)$ & $7252(4)$ & $10798(2)$ & $55(2)$ \\
\hline C(18) & $9377(3)$ & $7686(4)$ & $10781(2)$ & $53(2)$ \\
\hline C(19) & $9360(2)$ & $8508(4)$ & $10441(2)$ & $36(1)$ \\
\hline $\mathrm{C}(20)$ & $8238(2)$ & $12993(3)$ & $9157(2)$ & $34(1)$ \\
\hline $\mathrm{C}(21)$ & $8385(2)$ & $12409(3)$ & 8739(2) & $22(1)$ \\
\hline $\mathrm{C}(22)$ & $8897(2)$ & $11829(3)$ & $8861(2)$ & $20(1)$ \\
\hline $\mathrm{C}(23)$ & $9075(2)$ & $11345(3)$ & $8467(2)$ & $17(1)$ \\
\hline $\mathrm{C}(24)$ & $8722(2)$ & 11394(3) & $7935(1)$ & $16(1)$ \\
\hline$C(25)$ & $8888(2)$ & $10926(3)$ & $7490(2)$ & $22(1)$ \\
\hline $\mathrm{C}(26)$ & $9353(2)$ & 10109(3) & $7664(2)$ & $27(1)$ \\
\hline $\mathrm{C}(27)$ & $9046(2)$ & $11825(4)$ & $7183(2)$ & $36(1)$ \\
\hline $\mathrm{C}(28)$ & $8200(2)$ & $11944(3)$ & $7807(2)$ & 19(1) \\
\hline $\mathrm{C}(29)$ & $8038(2)$ & $12435(3)$ & $8203(2)$ & $23(1)$ \\
\hline $\mathrm{C}(30)$ & $6959(2)$ & $11639(3)$ & $8602(1)$ & $15(1)$ \\
\hline $\mathrm{C}(31)$ & $6944(2)$ & $11829(3)$ & $9114(2)$ & $18(1)$ \\
\hline $\mathrm{C}(32)$ & $7096(2)$ & $11027(3)$ & $9548(2)$ & $23(1)$ \\
\hline $\mathrm{C}(33)$ & $6777(2)$ & $12831(3)$ & $9225(2)$ & $23(1)$ \\
\hline $\mathrm{C}(34)$ & $6625(2)$ & $13627(3)$ & $8854(2)$ & $26(1)$ \\
\hline$C(35)$ & $6615(2)$ & $13418(3)$ & $8342(2)$ & $26(1)$ \\
\hline$C(36)$ & $6774(2)$ & $12434(3)$ & $8223(2)$ & $19(1)$ \\
\hline $\mathrm{C}(37)$ & $6768(1)$ & $10228(3)$ & $7738(1)$ & $14(1)$ \\
\hline $\mathrm{C}(38)$ & $6201(2)$ & $9927(3)$ & $7647(2)$ & 19(1) \\
\hline C(39) & $5941(2)$ & $9911(3)$ & $8080(2)$ & $25(1)$ \\
\hline $\mathrm{C}(40)$ & $5865(2)$ & $9662(3)$ & $7139(2)$ & $24(1)$ \\
\hline $\mathrm{C}(41)$ & $6065(2)$ & $9693(3)$ & $6717(2)$ & $23(1)$ \\
\hline $\mathrm{C}(42)$ & $6614(2)$ & $10012(3)$ & 6801(1) & $20(1)$ \\
\hline$C(43)$ & $6955(2)$ & $10275(3)$ & $7307(1)$ & 14(1) \\
\hline C12 & $9972(3)$ & 4946(4) & $9878(2)$ & $51(5)$ \\
\hline $\mathrm{C} 22$ & $9941(3)$ & $5524(4)$ & $10312(3)$ & $47(3)$ \\
\hline C32 & $9704(3)$ & $5069(6)$ & $10666(2)$ & $46(3)$ \\
\hline $\mathrm{C} 42$ & $9498(3)$ & $4035(6)$ & $10585(2)$ & $49(3)$ \\
\hline C52 & $9529(3)$ & $3457(4)$ & $10150(3)$ & $53(3)$ \\
\hline C62 & $9766(3)$ & $3912(4)$ & $9797(2)$ & $43(3)$ \\
\hline $\mathrm{C} 72$ & $10230(4)$ & $5440(7)$ & $9494(3)$ & $55(3)$ \\
\hline
\end{tabular}


Table S3. Anisotropic displacement parameters $\left(\AA^{2} \times 10^{3}\right)$ for $\mathbf{4 a}$. The anisotropic displacement factor exponent takes the form: $-2 \mathrm{p}^{2}\left[\mathrm{~h}^{2} \mathrm{a}^{* 2} \mathrm{U}^{11}+\ldots+2 \mathrm{~h} \mathrm{k} \mathrm{a}^{*} \mathrm{~b}^{*} \mathrm{U}^{12}\right]$

\begin{tabular}{|c|c|c|c|c|c|c|}
\hline & $\mathrm{U}^{11}$ & $\mathrm{U}^{22}$ & $\mathrm{U}^{33}$ & $\mathrm{U}^{23}$ & $\mathrm{U}^{13}$ & $\mathrm{U}^{12}$ \\
\hline $\operatorname{Ru}(1)$ & $13(1)$ & $10(1)$ & $13(1)$ & $-1(1)$ & $5(1)$ & $0(1)$ \\
\hline $\mathrm{S}(1)$ & $14(1)$ & $13(1)$ & $12(1)$ & $-1(1)$ & $3(1)$ & $0(1)$ \\
\hline$S(2)$ & $14(1)$ & $19(1)$ & $13(1)$ & $-4(1)$ & $2(1)$ & 1(1) \\
\hline $\mathrm{P}(1)$ & $11(1)$ & $11(1)$ & $13(1)$ & $-1(1)$ & $2(1)$ & $0(1)$ \\
\hline $\mathrm{P}(2)$ & $13(1)$ & $12(1)$ & $12(1)$ & $1(1)$ & $4(1)$ & $2(1)$ \\
\hline $\mathrm{O}(1)$ & $14(1)$ & $33(2)$ & $23(2)$ & $-11(1)$ & $4(1)$ & $-2(1)$ \\
\hline $\mathrm{O}(2)$ & $22(1)$ & $22(1)$ & $19(1)$ & $-5(1)$ & $5(1)$ & $4(1)$ \\
\hline $\mathrm{O}(3)$ & $14(1)$ & $15(1)$ & $16(1)$ & $3(1)$ & $5(1)$ & $3(1)$ \\
\hline $\mathrm{C}(1)$ & $13(2)$ & $12(2)$ & $12(2)$ & $-3(1)$ & $2(2)$ & $0(1)$ \\
\hline$C(2)$ & $12(2)$ & $15(2)$ & $20(2)$ & $0(2)$ & $3(2)$ & $5(1)$ \\
\hline $\mathrm{C}(3)$ & $16(2)$ & $33(2)$ & $37(2)$ & $-21(2)$ & $5(2)$ & $-2(2)$ \\
\hline $\mathrm{C}(4)$ & $15(2)$ & $71(4)$ & $75(4)$ & $-53(3)$ & $8(2)$ & $-7(2)$ \\
\hline $\mathrm{C}(5)$ & $16(2)$ & $67(4)$ & $72(4)$ & $-44(3)$ & $13(2)$ & $2(2)$ \\
\hline $\mathrm{C}(6)$ & $21(2)$ & $31(2)$ & $37(3)$ & $-18(2)$ & $7(2)$ & $4(2)$ \\
\hline $\mathrm{C}(7)$ & $15(2)$ & $19(2)$ & $23(2)$ & $-4(2)$ & $2(2)$ & $0(2)$ \\
\hline $\mathrm{C}(8)$ & $22(2)$ & $13(2)$ & $14(2)$ & $-5(2)$ & $5(2)$ & $-1(2)$ \\
\hline $\mathrm{C}(9)$ & $23(2)$ & $16(2)$ & $15(2)$ & $-3(2)$ & $1(2)$ & $0(2)$ \\
\hline$C(10)$ & $38(2)$ & $17(2)$ & $13(2)$ & $-1(2)$ & $4(2)$ & $3(2)$ \\
\hline $\mathrm{C}(11)$ & $40(2)$ & $13(2)$ & $24(2)$ & $2(2)$ & $18(2)$ & $1(2)$ \\
\hline$C(12)$ & $23(2)$ & $18(2)$ & $28(2)$ & $-4(2)$ & $14(2)$ & $-1(2)$ \\
\hline $\mathrm{C}(13)$ & $16(2)$ & $17(2)$ & $19(2)$ & $-1(2)$ & $4(2)$ & $3(2)$ \\
\hline$C(14)$ & $33(2)$ & $21(2)$ & $12(2)$ & $1(2)$ & $7(2)$ & $5(2)$ \\
\hline$C(15)$ & $52(3)$ & $30(2)$ & $30(2)$ & $0(2)$ & $27(2)$ & $5(2)$ \\
\hline$C(16)$ & $88(4)$ & $31(3)$ & $45(3)$ & $-2(2)$ & $50(3)$ & $-5(3)$ \\
\hline $\mathrm{C}(17)$ & $113(5)$ & $30(3)$ & $20(3)$ & $10(2)$ & $21(3)$ & $21(3)$ \\
\hline$C(18)$ & $73(4)$ & $40(3)$ & $30(3)$ & $5(2)$ & $-3(3)$ & $8(3)$ \\
\hline$C(19)$ & $41(3)$ & $33(3)$ & $23(2)$ & $-2(2)$ & $-4(2)$ & $9(2)$ \\
\hline$C(20)$ & $56(3)$ & $17(2)$ & $42(3)$ & $-8(2)$ & $34(2)$ & $-8(2)$ \\
\hline $\mathrm{C}(21)$ & $34(2)$ & $9(2)$ & $30(2)$ & $-5(2)$ & $21(2)$ & $-8(2)$ \\
\hline$C(22)$ & $26(2)$ & $16(2)$ & $14(2)$ & $-1(2)$ & $4(2)$ & $-9(2)$ \\
\hline $\mathrm{C}(23)$ & $13(2)$ & $14(2)$ & $24(2)$ & $0(2)$ & $4(2)$ & $-6(2)$ \\
\hline$C(24)$ & $19(2)$ & $11(2)$ & $21(2)$ & $-1(2)$ & $8(2)$ & $-4(2)$ \\
\hline$C(25)$ & $26(2)$ & $20(2)$ & $23(2)$ & $-3(2)$ & $13(2)$ & $-6(2)$ \\
\hline$C(26)$ & $25(2)$ & $28(2)$ & $36(2)$ & $-9(2)$ & $21(2)$ & $-6(2)$ \\
\hline $\mathrm{C}(27)$ & $50(3)$ & $33(3)$ & $34(3)$ & $-6(2)$ & $29(2)$ & $-11(2)$ \\
\hline $\mathrm{C}(28)$ & $21(2)$ & $17(2)$ & $19(2)$ & $1(2)$ & $7(2)$ & $-6(2)$ \\
\hline$C(29)$ & $27(2)$ & $14(2)$ & $34(2)$ & $5(2)$ & $17(2)$ & $-3(2)$ \\
\hline $\mathrm{C}(30)$ & $13(2)$ & $16(2)$ & $17(2)$ & $-1(2)$ & $6(2)$ & $-1(1)$ \\
\hline $\mathrm{C}(31)$ & $14(2)$ & $23(2)$ & $18(2)$ & $-2(2)$ & $6(2)$ & $-2(2)$ \\
\hline$C(32)$ & $24(2)$ & $32(2)$ & $15(2)$ & $0(2)$ & $9(2)$ & $1(2)$ \\
\hline $\mathrm{C}(33)$ & $17(2)$ & $28(2)$ & $27(2)$ & $-11(2)$ & $11(2)$ & $-4(2)$ \\
\hline$C(34)$ & $20(2)$ & $19(2)$ & $44(3)$ & $-4(2)$ & $15(2)$ & $2(2)$ \\
\hline$C(35)$ & $24(2)$ & $20(2)$ & $39(2)$ & $7(2)$ & $16(2)$ & $6(2)$ \\
\hline$C(36)$ & $21(2)$ & $17(2)$ & $21(2)$ & $2(2)$ & $8(2)$ & $3(2)$ \\
\hline $\mathrm{C}(37)$ & $13(2)$ & $10(2)$ & $16(2)$ & $2(1)$ & $2(2)$ & $3(1)$ \\
\hline $\mathrm{C}(38)$ & $17(2)$ & $17(2)$ & $23(2)$ & $4(2)$ & $7(2)$ & $3(2)$ \\
\hline$C(39)$ & $16(2)$ & $31(2)$ & $31(2)$ & $1(2)$ & $11(2)$ & $-4(2)$ \\
\hline $\mathrm{C}(40)$ & $13(2)$ & $25(2)$ & $29(2)$ & $2(2)$ & $1(2)$ & $-1(2)$ \\
\hline $\mathrm{C}(41)$ & $21(2)$ & $20(2)$ & $18(2)$ & $-1(2)$ & $-4(2)$ & $2(2)$ \\
\hline $\mathrm{C}(42)$ & $25(2)$ & $16(2)$ & $15(2)$ & $1(2)$ & $4(2)$ & $6(2)$ \\
\hline $\mathrm{C}(43)$ & $13(2)$ & $13(2)$ & $16(2)$ & $3(1)$ & $4(2)$ & $2(1)$ \\
\hline C12 & $18(5)$ & $64(8)$ & $54(14)$ & $27(10)$ & $-13(7)$ & $9(5)$ \\
\hline $\mathrm{C} 22$ & $26(6)$ & $44(8)$ & $57(8)$ & $-3(6)$ & $-7(5)$ & $9(5)$ \\
\hline C32 & $44(7)$ & $37(7)$ & $37(7)$ & $1(5)$ & $-13(5)$ & $13(5)$ \\
\hline $\mathrm{C} 42$ & $41(6)$ & $40(6)$ & $48(7)$ & $17(5)$ & $-10(5)$ & $7(5)$ \\
\hline C52 & $61(7)$ & $44(6)$ & $34(6)$ & $17(5)$ & $-9(5)$ & $-5(5)$ \\
\hline C62 & $25(5)$ & $39(6)$ & $45(6)$ & $-1(5)$ & $-13(5)$ & $2(4)$ \\
\hline C72 & $46(7)$ & $63(9)$ & $45(8)$ & $20(7)$ & $-1(6)$ & $12(7)$ \\
\hline
\end{tabular}




\subsection{Crystal Structure Determination of $\mathbf{4 b}$}

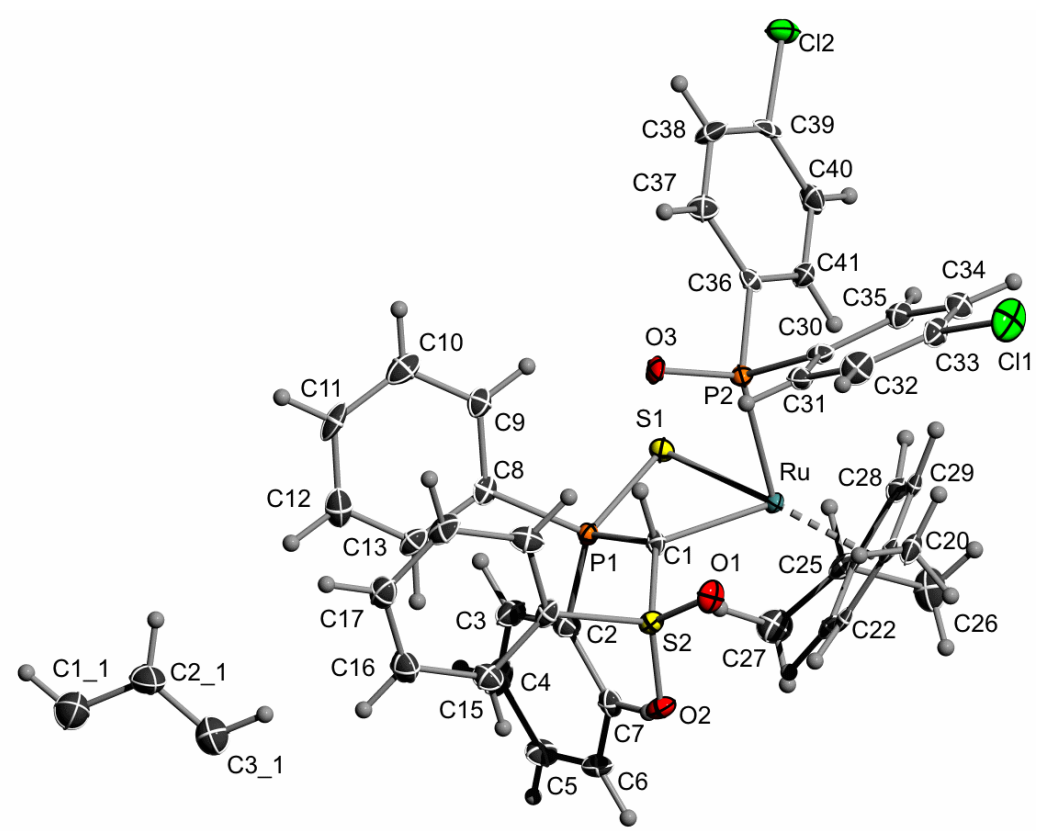

Figure. S19. ORTEP Plot of complex $\mathbf{4 b}$. Ellipsoids are drawn at the 50\% probability level.

Table S4. Atomic coordinates ( $\times 10^{4}$ ) and equivalent isotropic displacement parameters $\left(\AA^{2} \times 10^{3}\right)$ for $\mathbf{4 b}$. $\mathrm{U}(\mathrm{eq})$ is defined as one third of the trace of the orthogonalized $\mathrm{U}^{\mathrm{ij}}$ tensor.

\begin{tabular}{|c|c|c|c|c|}
\hline & $\mathrm{x}$ & $\mathrm{y}$ & $\mathrm{z}$ & $\mathrm{U}(\mathrm{eq})$ \\
\hline $\mathrm{Ru}(1)$ & $8343(1)$ & $9957(1)$ & $8516(1)$ & 11(1) \\
\hline $\mathrm{Cl}(1)$ & $6506(1)$ & $13688(1)$ & $8986(1)$ & $31(1)$ \\
\hline $\mathrm{S}(1)$ & $8207(1)$ & $8232(1)$ & $8093(1)$ & $14(1)$ \\
\hline $\mathrm{P}(1)$ & $8690(1)$ & $7813(1)$ & $8955(1)$ & $12(1)$ \\
\hline $\mathrm{O}(1)$ & $8678(1)$ & $10212(2)$ & $10084(1)$ & $18(1)$ \\
\hline $\mathrm{C}(1)$ & $8580(2)$ & $8942(3)$ & $9276(2)$ & $12(1)$ \\
\hline $\mathrm{Cl}(2)$ & $5524(1)$ & $8648(1)$ & $5778(1)$ & $24(1)$ \\
\hline $\mathrm{S}(2)$ & $8975(1)$ & $9343(1)$ & $10017(1)$ & $13(1)$ \\
\hline $\mathrm{P}(2)$ & $7445(1)$ & $9643(1)$ & $8406(1)$ & $13(1)$ \\
\hline $\mathrm{O}(2)$ & $9561(1)$ & $9477(2)$ & $10195(1)$ & $16(1)$ \\
\hline $\mathrm{C}(2)$ & $9382(2)$ & $7495(3)$ & $9086(2)$ & $15(1)$ \\
\hline $\mathrm{O}(3)$ & $7395(1)$ & $8838(2)$ & $8809(1)$ & $15(1)$ \\
\hline $\mathrm{C}(3)$ & $9434(2)$ & $6578(3)$ & $8835(2)$ & $22(1)$ \\
\hline $\mathrm{C}(4)$ & $9934(2)$ & $6324(3)$ & $8845(2)$ & $27(1)$ \\
\hline$C(5)$ & $10383(2)$ & $6984(3)$ & $9103(2)$ & $22(1)$ \\
\hline$C(6)$ & $10337(2)$ & $7889(3)$ & $9350(2)$ & $18(1)$ \\
\hline$C(7)$ & $9838(2)$ & $8149(3)$ & $9346(2)$ & $15(1)$ \\
\hline$C(8)$ & $8425(2)$ & $6704(3)$ & $9164(2)$ & $14(1)$ \\
\hline $\mathrm{C}(9)$ & $7848(2)$ & $6540(3)$ & $8886(2)$ & $17(1)$ \\
\hline$C(10)$ & $7629(2)$ & $5748(3)$ & $9076(2)$ & $24(1)$ \\
\hline $\mathrm{C}(11)$ & $7982(2)$ & $5122(3)$ & $9537(2)$ & $23(1)$ \\
\hline$C(12)$ & $8554(2)$ & $5281(3)$ & $9824(2)$ & $23(1)$ \\
\hline$C(13)$ & $8778(2)$ & $6063(3)$ & $9634(2)$ & $18(1)$ \\
\hline$C(14)$ & $8911(2)$ & $8332(3)$ & $10442(2)$ & $14(1)$ \\
\hline$C(15)$ & $9370(2)$ & $7736(3)$ & $10801(2)$ & $18(1)$ \\
\hline
\end{tabular}




\begin{tabular}{|c|c|c|c|c|}
\hline$C(16)$ & $9301(2)$ & $6919(3)$ & $11107(2)$ & $24(1)$ \\
\hline $\mathrm{C}(17)$ & $8780(2)$ & $6706(3)$ & $11053(2)$ & $22(1)$ \\
\hline$C(18)$ & $8324(2)$ & $7321(3)$ & $10703(2)$ & $22(1)$ \\
\hline C(19) & $8390(2)$ & $8142(3)$ & $10396(2)$ & $19(1)$ \\
\hline $\mathrm{C}(20)$ & $8318(2)$ & $12217(3)$ & $9196(2)$ & $21(1)$ \\
\hline $\mathrm{C}(21)$ & $8479(2)$ & $11604(3)$ & $8798(2)$ & $14(1)$ \\
\hline $\mathrm{C}(22)$ & $8996(2)$ & $11087(3)$ & $9032(2)$ & $15(1)$ \\
\hline $\mathrm{C}(23)$ & $9185(2)$ & 10597(3) & $8664(2)$ & $15(1)$ \\
\hline $\mathrm{C}(24)$ & $8834(2)$ & $10547(3)$ & $8041(2)$ & $14(1)$ \\
\hline $\mathrm{C}(25)$ & $9004(2)$ & $10068(3)$ & $7613(2)$ & $19(1)$ \\
\hline$C(26)$ & $9157(2)$ & 10923(3) & 7301(2) & $31(1)$ \\
\hline $\mathrm{C}(27)$ & $9472(2)$ & $9282(3)$ & $7892(2)$ & $25(1)$ \\
\hline $\mathrm{C}(28)$ & $8298(2)$ & $11020(3)$ & $7801(2)$ & $15(1)$ \\
\hline $\mathrm{C}(29)$ & $8128(2)$ & 11542(3) & $8167(2)$ & $16(1)$ \\
\hline $\mathrm{C}(30)$ & $7139(2)$ & $10805(3)$ & $8530(2)$ & $13(1)$ \\
\hline $\mathrm{C}(31)$ & $7190(2)$ & $10947(3)$ & $9096(2)$ & $16(1)$ \\
\hline $\mathrm{C}(32)$ & $6991(2)$ & $11827(3)$ & $9236(2)$ & $21(1)$ \\
\hline $\mathrm{C}(33)$ & $6744(2)$ & $12574(3)$ & $8807(2)$ & $19(1)$ \\
\hline $\mathrm{C}(34)$ & $6681(2)$ & $12460(3)$ & $8235(2)$ & 19(1) \\
\hline$C(35)$ & $6871(2)$ & 11564(3) & $8101(2)$ & $17(1)$ \\
\hline$C(36)$ & $6925(2)$ & $9309(3)$ & $7636(2)$ & $12(1)$ \\
\hline $\mathrm{C}(37)$ & $6461(2)$ & $8735(3)$ & $7558(2)$ & $20(1)$ \\
\hline $\mathrm{C}(38)$ & $6034(2)$ & $8518(3)$ & $6990(2)$ & $22(1)$ \\
\hline C(39) & $6074(2)$ & $8871(3)$ & $6497(2)$ & $16(1)$ \\
\hline $\mathrm{C}(40)$ & $6539(2)$ & $9398(3)$ & $6565(2)$ & $20(1)$ \\
\hline$C(41)$ & $6962(2)$ & $9615(3)$ & $7135(2)$ & $17(1)$ \\
\hline C1A1 & $9716(2)$ & $3429(4)$ & $12328(2)$ & $40(1)$ \\
\hline C2A1 & $9428(2)$ & $4344(4)$ & $12155(2)$ & $38(1)$ \\
\hline C3A1 & $9719(2)$ & $5254(3)$ & $12336(2)$ & $31(1)$ \\
\hline
\end{tabular}

Table S5. Anisotropic displacement parameters $\left(\AA^{2} \times 10^{3}\right)$ for $4 \mathbf{b}$. The anisotropic displacement factor exponent takes the form: $-2 \mathrm{p}^{2}\left[\mathrm{~h}^{2} \mathrm{a}^{* 2} \mathrm{U}^{11}+\ldots+2 \mathrm{~h} \mathrm{k} \mathrm{a}^{*} \mathrm{~b}^{*} \mathrm{U}^{12}\right]$.

\begin{tabular}{ccccccc}
\hline & $\mathrm{U}^{11}$ & $\mathrm{U}^{22}$ & $\mathrm{U}^{33}$ & $\mathrm{U}^{23}$ & $\mathrm{U}^{13}$ & $\mathrm{U}^{12}$ \\
\hline $\mathrm{Ru}(1)$ & $11(1)$ & $11(1)$ & $12(1)$ & $1(1)$ & $6(1)$ & $-1(1)$ \\
$\mathrm{Cl}(1)$ & $39(1)$ & $21(1)$ & $46(1)$ & $-8(1)$ & $31(1)$ & $3(1)$ \\
$\mathrm{S}(1)$ & $16(1)$ & $14(1)$ & $11(1)$ & $-1(1)$ & $6(1)$ & $-1(1)$ \\
$\mathrm{P}(1)$ & $12(1)$ & $11(1)$ & $12(1)$ & $-1(1)$ & $6(1)$ & $-1(1)$ \\
$\mathrm{O}(1)$ & $24(2)$ & $14(2)$ & $21(2)$ & $-5(1)$ & $14(1)$ & $0(1)$ \\
$\mathrm{C}(1)$ & $12(2)$ & $13(2)$ & $11(2)$ & $3(2)$ & $6(2)$ & $-1(2)$ \\
$\mathrm{Cl}(2)$ & $16(1)$ & $26(1)$ & $22(1)$ & $-5(1)$ & $3(1)$ & $-1(1)$ \\
$\mathrm{S}(2)$ & $14(1)$ & $14(1)$ & $12(1)$ & $-1(1)$ & $6(1)$ & $-2(1)$ \\
$\mathrm{P}(2)$ & $12(1)$ & $13(1)$ & $15(1)$ & $1(1)$ & $7(1)$ & $0(1)$ \\
$\mathrm{O}(2)$ & $13(2)$ & $21(2)$ & $15(1)$ & $-3(1)$ & $7(1)$ & $-5(1)$ \\
$\mathrm{C}(2)$ & $13(2)$ & $17(2)$ & $16(2)$ & $2(2)$ & $9(2)$ & $1(2)$ \\
$\mathrm{O}(3)$ & $16(2)$ & $15(1)$ & $19(2)$ & $6(1)$ & $11(1)$ & $-1(1)$ \\
$\mathrm{C}(3)$ & $20(2)$ & $20(2)$ & $28(2)$ & $-5(2)$ & $13(2)$ & $-5(2)$ \\
$\mathrm{C}(4)$ & $30(3)$ & $16(2)$ & $43(3)$ & $-8(2)$ & $25(2)$ & $-1(2)$ \\
$\mathrm{C}(5)$ & $15(2)$ & $26(2)$ & $28(2)$ & $4(2)$ & $14(2)$ & $6(2)$
\end{tabular}




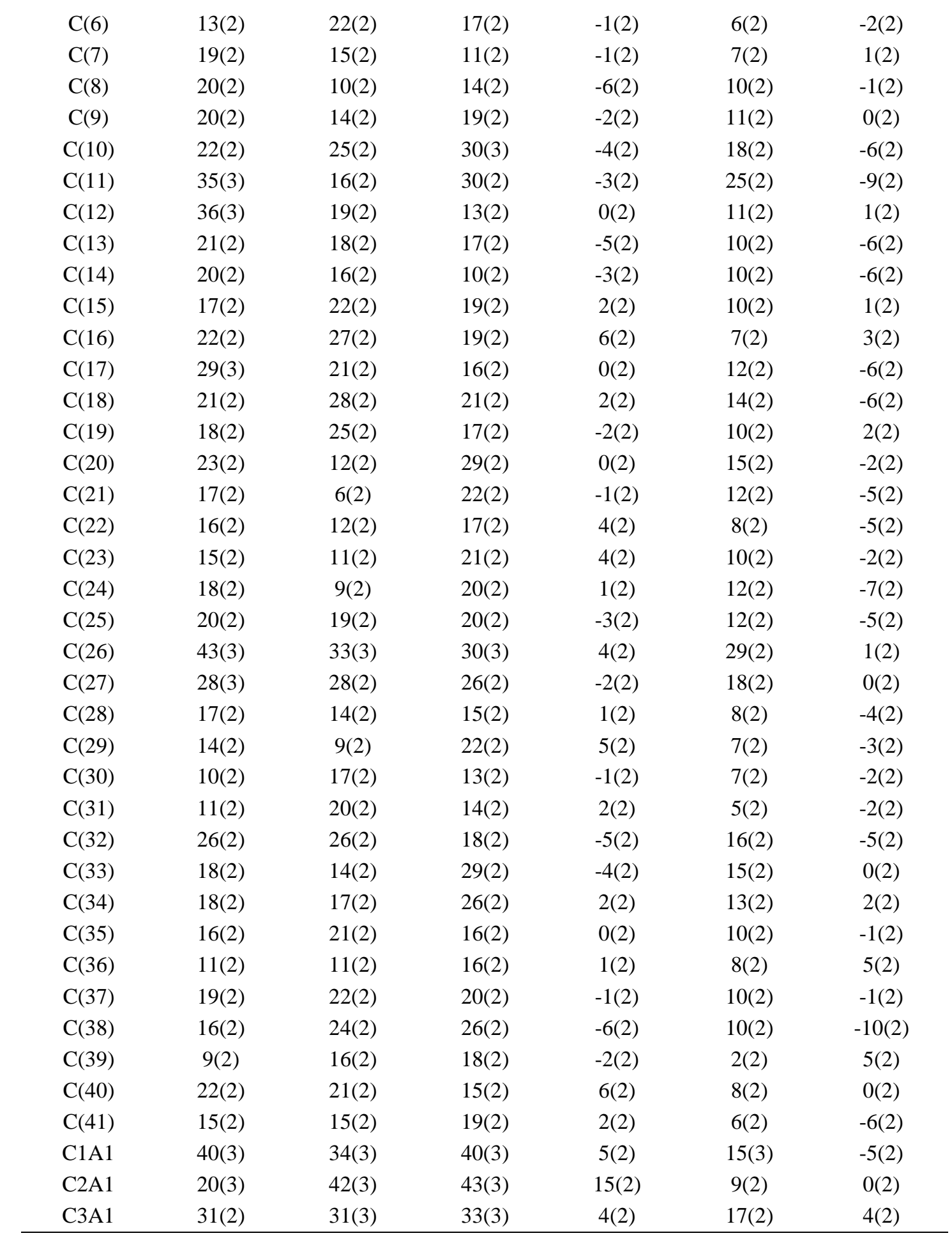


3.3 Crystal Structure Determination of 5a

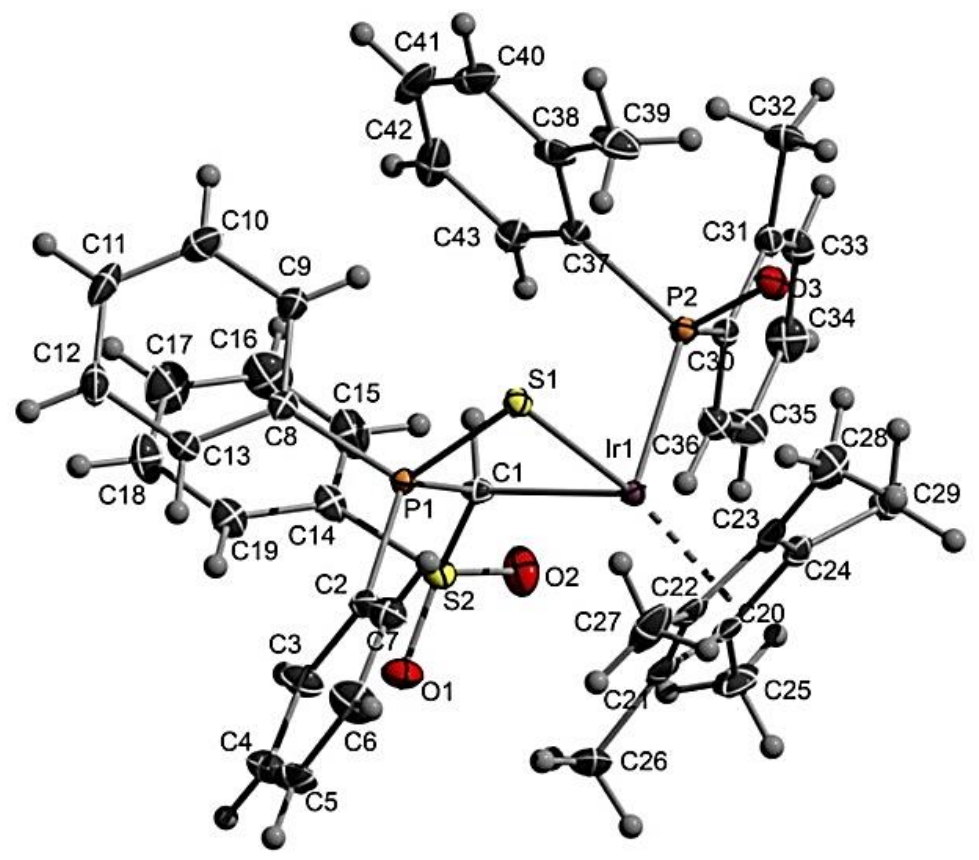

Figure S20. ORTEP Plot of complex 5a. Ellipsoids are drawn at the 50\% probability level.

Table S6. Atomic coordinates ( x 10 $)$ and equivalent isotropic displacement parameters $\left(\AA^{2} \times 10^{3}\right)$ for $\mathbf{5 a}$. $\mathrm{U}(\mathrm{eq})$ is defined as one third of the trace of the orthogonalized $\mathrm{U}^{\mathrm{ij}}$ tensor.

\begin{tabular}{|c|c|c|c|c|}
\hline & $\mathrm{X}$ & $\mathrm{y}$ & $\mathrm{Z}$ & $\mathrm{U}(\mathrm{eq})$ \\
\hline $\operatorname{Ir}(1)$ & $1859(1)$ & $1312(1)$ & $8491(1)$ & $12(1)$ \\
\hline $\mathrm{S}(1)$ & 2992(1) & $360(1)$ & $9141(1)$ & $14(1)$ \\
\hline $\mathrm{P}(1)$ & $4333(1)$ & $870(1)$ & $8748(1)$ & $12(1)$ \\
\hline $\mathrm{O}(1)$ & $4631(2)$ & $2447(1)$ & $7835(1)$ & $25(1)$ \\
\hline $\mathrm{C}(1)$ & $3450(2)$ & $1265(1)$ & $7922(2)$ & $14(1)$ \\
\hline$S(2)$ & $3971(1)$ & $1940(1)$ & $7353(1)$ & $18(1)$ \\
\hline $\mathrm{P}(2)$ & 879(1) & $506(1)$ & $7643(1)$ & $13(1)$ \\
\hline $\mathrm{O}(2)$ & $3018(2)$ & 2189(1) & $6818(1)$ & $27(1)$ \\
\hline$C(2)$ & $5089(2)$ & $1442(1)$ & $9485(2)$ & $16(1)$ \\
\hline $\mathrm{O}(3)$ & $-126(2)$ & 188(1) & $8002(1)$ & $20(1)$ \\
\hline$C(3)$ & $5895(3)$ & $1945(2)$ & $9305(2)$ & $22(1)$ \\
\hline $\mathrm{C}(4)$ & $6520(3)$ & $2320(2)$ & $9896(2)$ & $25(1)$ \\
\hline $\mathrm{C}(5)$ & $6349(3)$ & $2207(2)$ & $10669(2)$ & $25(1)$ \\
\hline$C(6)$ & $5580(3)$ & $1704(2)$ & $10853(2)$ & $29(1)$ \\
\hline$C(7)$ & $4955(3)$ & $1319(2)$ & $10267(2)$ & $21(1)$ \\
\hline $\mathrm{C}(8)$ & $5436(2)$ & 285(1) & $8459(2)$ & $14(1)$ \\
\hline $\mathrm{C}(9)$ & $5064(3)$ & $-246(2)$ & $7933(2)$ & $20(1)$ \\
\hline$C(10)$ & $5867(3)$ & $-689(2)$ & $7652(2)$ & $26(1)$ \\
\hline $\mathrm{C}(11)$ & $7027(3)$ & $-600(2)$ & $7885(2)$ & $27(1)$ \\
\hline$C(12)$ & $7408(3)$ & $-83(2)$ & $8413(2)$ & $26(1)$ \\
\hline$C(13)$ & $6613(2)$ & $358(2)$ & $8709(2)$ & $19(1)$ \\
\hline $\mathrm{C}(14)$ & $4924(2)$ & $1501(2)$ & $6770(2)$ & $18(1)$ \\
\hline$C(15)$ & $4469(3)$ & $1258(2)$ & $6041(2)$ & $28(1)$ \\
\hline$C(16)$ & $5183(3)$ & $887(2)$ & $5591(2)$ & $35(1)$ \\
\hline$C(17)$ & $6327(3)$ & $765(2)$ & $5875(2)$ & $36(1)$ \\
\hline $\mathrm{C}(18)$ & $6777(3)$ & $1011(2)$ & $6601(2)$ & $28(1)$ \\
\hline$C(19)$ & $6075(3)$ & $1388(2)$ & $7057(2)$ & $22(1)$ \\
\hline $\mathrm{C}(20)$ & $1123(2)$ & $2377(1)$ & $8432(2)$ & 19(1) \\
\hline $\mathrm{C}(21)$ & 1976(3) & $2372(2)$ & $9127(2)$ & $22(1)$ \\
\hline
\end{tabular}




\begin{tabular}{lrrrr}
$\mathrm{C}(22)$ & $1630(3)$ & $1864(2)$ & $9650(2)$ & $22(1)$ \\
$\mathrm{C}(23)$ & $565(3)$ & $1538(2)$ & $9299(2)$ & $19(1)$ \\
$\mathrm{C}(24)$ & $228(2)$ & $1888(2)$ & $8570(2)$ & $19(1)$ \\
$\mathrm{C}(25)$ & $1054(3)$ & $2918(2)$ & $7804(2)$ & $30(1)$ \\
$\mathrm{C}(26)$ & $2966(3)$ & $2872(2)$ & $9271(2)$ & $35(1)$ \\
$\mathrm{C}(27)$ & $2191(3)$ & $1687(2)$ & $10454(2)$ & $34(1)$ \\
$\mathrm{C}(28)$ & $-146(3)$ & $1021(2)$ & $9692(2)$ & $29(1)$ \\
$\mathrm{C}(29)$ & $-932(3)$ & $1805(2)$ & $8097(2)$ & $24(1)$ \\
$\mathrm{C}(30)$ & $312(2)$ & $855(2)$ & $6662(2)$ & $15(1)$ \\
$\mathrm{C}(31)$ & $-409(2)$ & $439(2)$ & $6119(2)$ & $16(1)$ \\
$\mathrm{C}(32)$ & $-771(3)$ & $-296(2)$ & $6295(2)$ & $21(1)$ \\
$\mathrm{C}(33)$ & $-787(3)$ & $726(2)$ & $5383(2)$ & $22(1)$ \\
$\mathrm{C}(34)$ & $-510(3)$ & $1402(2)$ & $5185(2)$ & $26(1)$ \\
$\mathrm{C}(35)$ & $173(3)$ & $1805(2)$ & $5721(2)$ & $27(1)$ \\
$\mathrm{C}(36)$ & $591(3)$ & $1526(2)$ & $6448(2)$ & $20(1)$ \\
$\mathrm{C}(37)$ & $1798(2)$ & $-205(1)$ & $7308(2)$ & $15(1)$ \\
$\mathrm{C}(38)$ & $1888(3)$ & $-874(2)$ & $7651(2)$ & $22(1)$ \\
$\mathrm{C}(39)$ & $1298(3)$ & $-1072(2)$ & $8359(2)$ & $31(1)$ \\
$\mathrm{C}(40)$ & $2542(3)$ & $-1381(2)$ & $7310(2)$ & $30(1)$ \\
$\mathrm{C}(41)$ & $3120(3)$ & $-1241(2)$ & $6674(2)$ & $34(1)$ \\
$\mathrm{C}(42)$ & $3054(3)$ & $-583(2)$ & $6335(2)$ & $29(1)$ \\
$\mathrm{C}(43)$ & $2388(2)$ & $-73(2)$ & $6651(2)$ & $20(1)$ \\
\hline
\end{tabular}

Table S7. Anisotropic displacement parameters $\left(\AA^{2} \times 10^{3}\right)$ for 5a. The anisotropicdisplacement factor exponent takes the form: $-2 \mathrm{p}^{2}\left[\mathrm{~h}^{2} \mathrm{a}^{* 2} \mathrm{U}^{11}+\ldots+2 \mathrm{~h} \mathrm{k} \mathrm{a}^{*} \mathrm{~b}^{*} \mathrm{U}^{12}\right]$.

\begin{tabular}{|c|c|c|c|c|c|c|}
\hline & $\mathrm{U}^{11}$ & $\mathrm{U}^{22}$ & $\mathrm{U}^{33}$ & $\mathrm{U}^{23}$ & $\mathrm{U}^{13}$ & $\mathrm{U}^{12}$ \\
\hline $\operatorname{Ir}(1)$ & $12(1)$ & $11(1)$ & $13(1)$ & $-2(1)$ & $0(1)$ & 1(1) \\
\hline$S(1)$ & $13(1)$ & $14(1)$ & $14(1)$ & 1(1) & $2(1)$ & $0(1)$ \\
\hline $\mathrm{P}(1)$ & $12(1)$ & 11(1) & $13(1)$ & $0(1)$ & 1(1) & $0(1)$ \\
\hline $\mathrm{O}(1)$ & $27(1)$ & $17(1)$ & $32(1)$ & $0(1)$ & $3(1)$ & $-5(1)$ \\
\hline $\mathrm{C}(1)$ & $16(2)$ & $12(2)$ & $14(2)$ & 1(1) & $0(1)$ & $-1(1)$ \\
\hline$S(2)$ & $17(1)$ & $17(1)$ & $19(1)$ & $6(1)$ & 1(1) & $-1(1)$ \\
\hline $\mathrm{P}(2)$ & $13(1)$ & $14(1)$ & $13(1)$ & $-2(1)$ & $2(1)$ & $-1(1)$ \\
\hline $\mathrm{O}(2)$ & $21(1)$ & $30(1)$ & $29(1)$ & $17(1)$ & $0(1)$ & $3(1)$ \\
\hline$C(2)$ & $16(2)$ & $13(2)$ & $17(2)$ & $-2(1)$ & $-2(1)$ & 1(1) \\
\hline $\mathrm{O}(3)$ & $20(1)$ & $21(1)$ & $19(1)$ & $-4(1)$ & $7(1)$ & $-4(1)$ \\
\hline$C(3)$ & $26(2)$ & $23(2)$ & $17(2)$ & 1(1) & $-1(1)$ & $-4(1)$ \\
\hline $\mathrm{C}(4)$ & $26(2)$ & $19(2)$ & $28(2)$ & $1(1)$ & $-4(1)$ & $-6(1)$ \\
\hline$C(5)$ & $27(2)$ & $21(2)$ & $25(2)$ & $-7(1)$ & $-10(1)$ & $-2(1)$ \\
\hline$C(6)$ & $35(2)$ & $35(2)$ & $14(2)$ & $0(1)$ & $-5(1)$ & $-4(2)$ \\
\hline$C(7)$ & $22(2)$ & $19(2)$ & $21(2)$ & $3(1)$ & $-1(1)$ & $-1(1)$ \\
\hline $\mathrm{C}(8)$ & $17(2)$ & $13(2)$ & $13(2)$ & $4(1)$ & $4(1)$ & 1(1) \\
\hline $\mathrm{C}(9)$ & $17(2)$ & $20(2)$ & $23(2)$ & $-1(1)$ & 1(1) & $3(1)$ \\
\hline$C(10)$ & $33(2)$ & $21(2)$ & $24(2)$ & $-5(1)$ & $3(1)$ & $5(1)$ \\
\hline $\mathrm{C}(11)$ & $26(2)$ & $25(2)$ & $32(2)$ & $4(1)$ & $15(2)$ & $13(1)$ \\
\hline$C(12)$ & $15(2)$ & $28(2)$ & $37(2)$ & $6(2)$ & $5(1)$ & $2(1)$ \\
\hline$C(13)$ & $17(2)$ & $16(2)$ & $24(2)$ & $-1(1)$ & $3(1)$ & $-2(1)$ \\
\hline$C(14)$ & $18(2)$ & $22(2)$ & $15(2)$ & $7(1)$ & $4(1)$ & $-5(1)$ \\
\hline$C(15)$ & $24(2)$ & $39(2)$ & $21(2)$ & $9(1)$ & $2(1)$ & $-4(2)$ \\
\hline$C(16)$ & $38(2)$ & $46(2)$ & $20(2)$ & $-3(2)$ & $4(2)$ & $-6(2)$ \\
\hline$C(17)$ & $37(2)$ & $48(2)$ & $26(2)$ & $-3(2)$ & $14(2)$ & $-1(2)$ \\
\hline$C(18)$ & $20(2)$ & $40(2)$ & $26(2)$ & $4(2)$ & $7(1)$ & $-1(2)$ \\
\hline$C(19)$ & $22(2)$ & $26(2)$ & $17(2)$ & $4(1)$ & $4(1)$ & $-4(1)$ \\
\hline $\mathrm{C}(20)$ & $22(2)$ & $9(2)$ & $25(2)$ & $-4(1)$ & $4(1)$ & $8(1)$ \\
\hline $\mathrm{C}(21)$ & $23(1)$ & $18(1)$ & $23(1)$ & $-13(1)$ & 1(1) & $7(1)$ \\
\hline$C(22)$ & $23(1)$ & $18(1)$ & $23(1)$ & $-13(1)$ & $1(1)$ & $7(1)$ \\
\hline $\mathrm{C}(23)$ & $20(2)$ & $20(2)$ & $17(2)$ & $-7(1)$ & $4(1)$ & $7(1)$ \\
\hline $\mathrm{C}(24)$ & $18(2)$ & $17(2)$ & $20(2)$ & $-6(1)$ & $1(1)$ & $6(1)$ \\
\hline
\end{tabular}




\begin{tabular}{llllccc}
$\mathrm{C}(25)$ & $37(2)$ & $12(2)$ & $41(2)$ & $4(1)$ & $6(2)$ & $6(1)$ \\
$\mathrm{C}(26)$ & $28(2)$ & $22(2)$ & $52(2)$ & $-18(2)$ & $-1(2)$ & $-2(1)$ \\
$\mathrm{C}(27)$ & $37(2)$ & $42(2)$ & $21(2)$ & $-14(2)$ & $-5(2)$ & $19(2)$ \\
$\mathrm{C}(28)$ & $32(2)$ & $31(2)$ & $26(2)$ & $0(1)$ & $14(2)$ & $2(2)$ \\
$\mathrm{C}(29)$ & $19(2)$ & $26(2)$ & $27(2)$ & $-5(1)$ & $0(1)$ & $8(1)$ \\
$\mathrm{C}(30)$ & $11(2)$ & $19(2)$ & $14(2)$ & $-2(1)$ & $1(1)$ & $2(1)$ \\
$\mathrm{C}(31)$ & $13(2)$ & $19(2)$ & $17(2)$ & $-1(1)$ & $1(1)$ & $1(1)$ \\
$\mathrm{C}(32)$ & $24(2)$ & $20(2)$ & $18(2)$ & $-6(1)$ & $-2(1)$ & $-5(1)$ \\
$\mathrm{C}(33)$ & $18(2)$ & $29(2)$ & $18(2)$ & $-4(1)$ & $0(1)$ & $1(1)$ \\
$\mathrm{C}(34)$ & $27(2)$ & $35(2)$ & $15(2)$ & $6(1)$ & $-1(1)$ & $3(1)$ \\
$\mathrm{C}(35)$ & $31(2)$ & $22(2)$ & $27(2)$ & $8(1)$ & $2(1)$ & $-3(1)$ \\
$\mathrm{C}(36)$ & $18(2)$ & $20(2)$ & $22(2)$ & $0(1)$ & $2(1)$ & $-2(1)$ \\
$\mathrm{C}(37)$ & $15(2)$ & $17(2)$ & $11(2)$ & $-6(1)$ & $-5(1)$ & $1(1)$ \\
$\mathrm{C}(38)$ & $23(2)$ & $16(2)$ & $25(2)$ & $-3(1)$ & $-11(1)$ & $-3(1)$ \\
$\mathrm{C}(39)$ & $37(2)$ & $18(2)$ & $36(2)$ & $7(1)$ & $-7(2)$ & $-10(2)$ \\
$\mathrm{C}(40)$ & $34(2)$ & $18(2)$ & $34(2)$ & $-7(1)$ & $-15(2)$ & $6(1)$ \\
$\mathrm{C}(41)$ & $31(2)$ & $36(2)$ & $33(2)$ & $-23(2)$ & $-13(2)$ & $18(2)$ \\
$\mathrm{C}(42)$ & $21(2)$ & $49(2)$ & $15(2)$ & $-12(1)$ & $-4(1)$ & $9(2)$ \\
$\mathrm{C}(43)$ & $20(2)$ & $25(2)$ & $13(2)$ & $-6(1)$ & $-2(1)$ & $3(1)$ \\
\hline
\end{tabular}

\subsection{Crystal Structure Determination of $\mathbf{5 b}$}

All hydrogen atoms were refined on ideal positions except for $\mathrm{H} 1$ at $\mathrm{C} 1$, which was found in the difference Fourier map and refined independently.

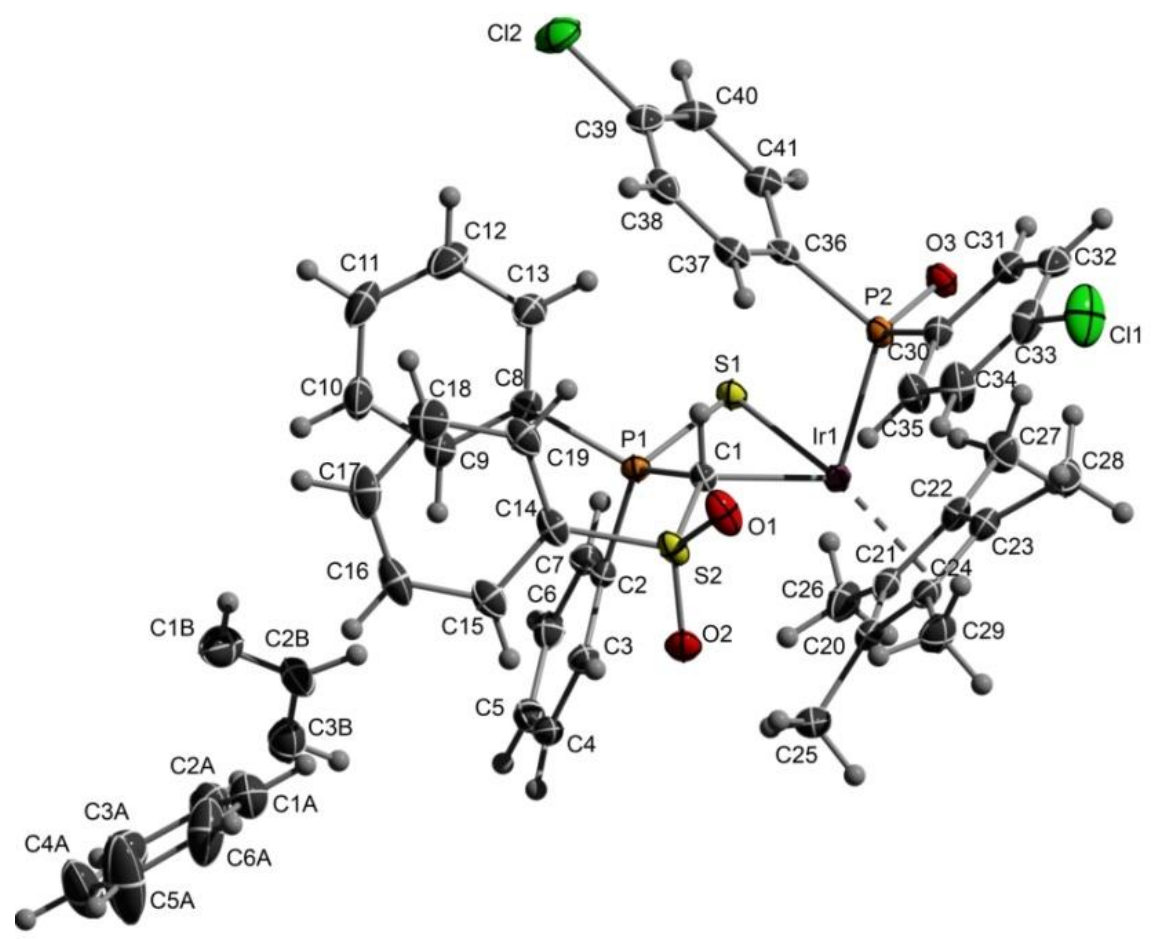

Figure S21. ORTEP Plot of complex 5b. Ellipsoids are drawn at the 50\% probability level. 


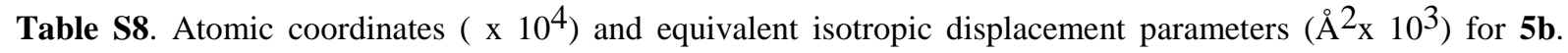
$\mathrm{U}(\mathrm{eq})$ is defined as one third of the trace of the orthogonalized $\mathrm{U}^{\mathrm{ij}}$ tensor.

\begin{tabular}{|c|c|c|c|c|}
\hline & $\mathrm{X}$ & $\mathrm{y}$ & $\mathrm{Z}$ & $\mathrm{U}(\mathrm{eq})$ \\
\hline $\operatorname{Ir}(1)$ & $6533(1)$ & $4410(1)$ & $8379(1)$ & $16(1)$ \\
\hline $\mathrm{Cl}(1)$ & $10160(1)$ & $1874(1)$ & 8144(1) & $46(1)$ \\
\hline $\mathrm{Cl}(2)$ & 3394(1) & $1950(1)$ & $10218(1)$ & $39(1)$ \\
\hline $\mathrm{S}(1)$ & $5029(1)$ & $4532(1)$ & $9211(1)$ & $19(1)$ \\
\hline$S(2)$ & $5334(1)$ & $3699(1)$ & $6503(1)$ & 21(1) \\
\hline $\mathrm{P}(1)$ & 4293(1) & $4370(1)$ & 8003(1) & $17(1)$ \\
\hline $\mathrm{P}(2)$ & $6948(1)$ & $3675(1)$ & $9360(1)$ & $18(1)$ \\
\hline $\mathrm{O}(1)$ & $6041(1)$ & $3225(1)$ & $6523(1)$ & $29(1)$ \\
\hline $\mathrm{O}(2)$ & $5495(1)$ & $4164(1)$ & 5902(1) & $26(1)$ \\
\hline $\mathrm{O}(3)$ & $7356(1)$ & $3874(1)$ & $10282(1)$ & $23(1)$ \\
\hline $\mathrm{C}(1)$ & $5308(2)$ & $3938(1)$ & $7616(2)$ & $16(1)$ \\
\hline $\mathrm{C}(2)$ & $3904(2)$ & $5021(1)$ & $7426(2)$ & $18(1)$ \\
\hline $\mathrm{C}(3)$ & $3448(2)$ & $5422(1)$ & $7957(2)$ & $22(1)$ \\
\hline $\mathrm{C}(4)$ & 2992(2) & $5906(1)$ & $7573(2)$ & $25(1)$ \\
\hline $\mathrm{C}(5)$ & $2995(2)$ & 5994(1) & $6660(2)$ & $26(1)$ \\
\hline$C(6)$ & $3466(2)$ & $5607(1)$ & $6132(2)$ & $24(1)$ \\
\hline$C(7)$ & $3911(2)$ & $5115(1)$ & $6510(2)$ & 21(1) \\
\hline $\mathrm{C}(8)$ & $3089(2)$ & $3995(1)$ & $8090(2)$ & 21(1) \\
\hline $\mathrm{C}(9)$ & $2197(2)$ & $4132(1)$ & $7552(2)$ & $26(1)$ \\
\hline$C(10)$ & $1297(2)$ & 3831(1) & $7635(2)$ & $35(1)$ \\
\hline $\mathrm{C}(11)$ & $1283(2)$ & $3398(1)$ & $8253(2)$ & $39(1)$ \\
\hline $\mathrm{C}(12)$ & $2165(2)$ & $3256(1)$ & $8787(2)$ & $36(1)$ \\
\hline$C(13)$ & $3068(2)$ & $3554(1)$ & $8708(2)$ & $27(1)$ \\
\hline$C(14)$ & $4083(2)$ & $3426(1)$ & $6199(2)$ & $21(1)$ \\
\hline$C(15)$ & $3727(2)$ & $2965(1)$ & $6664(2)$ & $27(1)$ \\
\hline$C(16)$ & $2738(2)$ & $2765(1)$ & $6446(2)$ & $34(1)$ \\
\hline$C(17)$ & $2120(2)$ & $3022(1)$ & $5760(2)$ & $34(1)$ \\
\hline$C(18)$ & $2485(2)$ & $3469(1)$ & $5289(2)$ & $32(1)$ \\
\hline C(19) & $3466(2)$ & $3680(1)$ & $5508(2)$ & $26(1)$ \\
\hline $\mathrm{C}(20)$ & $7591(2)$ & $5075(1)$ & $8926(2)$ & $22(1)$ \\
\hline $\mathrm{C}(21)$ & $6887(2)$ & $5356(1)$ & $8267(2)$ & $23(1)$ \\
\hline $\mathrm{C}(22)$ & $6989(2)$ & $5115(1)$ & $7416(2)$ & $23(1)$ \\
\hline $\mathrm{C}(23)$ & $7746(2)$ & $4662(1)$ & $7535(2)$ & $22(1)$ \\
\hline $\mathrm{C}(24)$ & $8165(2)$ & $4671(1)$ & $8455(2)$ & $22(1)$ \\
\hline$C(25)$ & $7810(2)$ & $5243(1)$ & $9890(2)$ & $30(1)$ \\
\hline$C(26)$ & $6202(2)$ & 5841(1) & $8482(2)$ & $30(1)$ \\
\hline $\mathrm{C}(27)$ & $6527(2)$ & $5335(1)$ & $6532(2)$ & $32(1)$ \\
\hline $\mathrm{C}(28)$ & $8174(2)$ & $4343(1)$ & $6787(2)$ & $32(1)$ \\
\hline C(29) & $9100(2)$ & $4367(1)$ & $8845(2)$ & $28(1)$ \\
\hline $\mathrm{C}(30)$ & $7876(2)$ & $3169(1)$ & $8964(2)$ & 21(1) \\
\hline $\mathrm{C}(31)$ & $7886(2)$ & $3013(1)$ & $8071(2)$ & $27(1)$ \\
\hline$C(32)$ & $8576(2)$ & $2608(1)$ & $7818(2)$ & $34(1)$ \\
\hline $\mathrm{C}(33)$ & $9269(2)$ & $2369(1)$ & $8460(2)$ & $29(1)$ \\
\hline $\mathrm{C}(34)$ & $9291(2)$ & $2525(1)$ & $9351(2)$ & $28(1)$ \\
\hline $\mathrm{C}(35)$ & $8590(2)$ & 2921(1) & $9596(2)$ & $25(1)$ \\
\hline$C(36)$ & $5905(2)$ & $3167(1)$ & $9529(2)$ & $19(1)$ \\
\hline$C(37)$ & $5586(2)$ & $2719(1)$ & $8949(2)$ & $22(1)$ \\
\hline $\mathrm{C}(38)$ & $4820(2)$ & $2342(1)$ & $9151(2)$ & $25(1)$ \\
\hline C(39) & $4369(2)$ & 2413(1) & $9942(2)$ & $25(1)$ \\
\hline $\mathrm{C}(40)$ & $4669(2)$ & $2847(1)$ & $10532(2)$ & $27(1)$ \\
\hline $\mathrm{C}(41)$ & $5435(2)$ & $3219(1)$ & $10327(2)$ & $22(1)$ \\
\hline $\mathrm{C} 11$ & $8719(3)$ & $6707(2)$ & $8134(3)$ & $56(1)$ \\
\hline $\mathrm{C} 41$ & $10016(3)$ & $5852(2)$ & $7655(2)$ & $45(1)$ \\
\hline C51 & $10220(3)$ & $6132(2)$ & $8448(3)$ & $63(1)$ \\
\hline C61 & $9556(3)$ & $6554(2)$ & $8692(3)$ & $86(2)$ \\
\hline $\mathrm{C} 21$ & $8511(3)$ & $6426(2)$ & $7347(2)$ & $45(1)$ \\
\hline C31 & $9155(3)$ & $6000(2)$ & $7104(2)$ & $45(1)$ \\
\hline
\end{tabular}




\begin{tabular}{rrrrr}
$\mathrm{C} 12$ & $10225(3)$ & $4438(1)$ & $5164(2)$ & $41(1)$ \\
$\mathrm{C} 22$ & $9286(3)$ & $4589(2)$ & $4748(2)$ & $39(1)$ \\
$\mathrm{C} 32$ & $9058(3)$ & $5151(2)$ & $4588(2)$ & $42(1)$ \\
\hline
\end{tabular}

Table S9. Anisotropic displacement parameters $\left(\AA^{2} \times 10^{3}\right)$ for $\mathbf{5 b}$. The anisotropic displacement factor exponent takes the form: $-2 p^{2}\left[h^{2} a^{* 2} U^{11}+\ldots+2 h k a^{*} b^{*} U^{12}\right]$

\begin{tabular}{|c|c|c|c|c|c|c|}
\hline & $\mathrm{U}^{11}$ & $\mathrm{U}^{22}$ & $\mathrm{U}^{33}$ & $\mathrm{U}^{23}$ & $\mathrm{U}^{13}$ & $\mathrm{U}^{12}$ \\
\hline $\operatorname{Ir}(1)$ & $14(1)$ & 19(1) & $15(1)$ & $-1(1)$ & $2(1)$ & $1(1)$ \\
\hline $\mathrm{Cl}(1)$ & $31(1)$ & $35(1)$ & $73(1)$ & $-17(1)$ & $3(1)$ & $12(1)$ \\
\hline $\mathrm{Cl}(2)$ & $46(1)$ & $30(1)$ & $42(1)$ & $3(1)$ & $10(1)$ & $-15(1)$ \\
\hline $\mathrm{S}(1)$ & $19(1)$ & $23(1)$ & $15(1)$ & $-2(1)$ & $2(1)$ & $0(1)$ \\
\hline$S(2)$ & $21(1)$ & $26(1)$ & $15(1)$ & $-4(1)$ & $0(1)$ & $4(1)$ \\
\hline $\mathrm{P}(1)$ & $17(1)$ & $18(1)$ & $15(1)$ & $-1(1)$ & $2(1)$ & $0(1)$ \\
\hline $\mathrm{P}(2)$ & $18(1)$ & $22(1)$ & $15(1)$ & $-1(1)$ & $0(1)$ & $2(1)$ \\
\hline $\mathrm{O}(1)$ & $27(1)$ & $34(1)$ & $25(1)$ & $-9(1)$ & $-2(1)$ & $14(1)$ \\
\hline $\mathrm{O}(2)$ & $26(1)$ & $35(1)$ & $16(1)$ & $0(1)$ & $5(1)$ & $-1(1)$ \\
\hline $\mathrm{O}(3)$ & $26(1)$ & $28(1)$ & $16(1)$ & $-2(1)$ & $-2(1)$ & $0(1)$ \\
\hline $\mathrm{C}(1)$ & $15(1)$ & $17(1)$ & $15(1)$ & $-1(1)$ & $-1(1)$ & $0(1)$ \\
\hline$C(2)$ & $14(1)$ & $20(1)$ & $20(1)$ & $1(1)$ & $1(1)$ & $-3(1)$ \\
\hline$C(3)$ & $22(2)$ & $24(2)$ & $18(1)$ & $-2(1)$ & $3(1)$ & $0(1)$ \\
\hline $\mathrm{C}(4)$ & $22(2)$ & $23(2)$ & $30(2)$ & $-3(1)$ & $1(1)$ & $0(1)$ \\
\hline$C(5)$ & $22(2)$ & $23(2)$ & $33(2)$ & $5(1)$ & $-5(1)$ & $-1(1)$ \\
\hline$C(6)$ & $24(2)$ & $28(2)$ & $20(2)$ & $5(1)$ & $0(1)$ & $-7(1)$ \\
\hline$C(7)$ & $19(1)$ & $24(2)$ & $19(1)$ & $0(1)$ & $3(1)$ & $0(1)$ \\
\hline $\mathrm{C}(8)$ & $22(2)$ & $21(2)$ & $19(1)$ & $-4(1)$ & $4(1)$ & $0(1)$ \\
\hline $\mathrm{C}(9)$ & $22(2)$ & $28(2)$ & $27(2)$ & $1(1)$ & $2(1)$ & $-2(1)$ \\
\hline$C(10)$ & $18(2)$ & $38(2)$ & $46(2)$ & $2(2)$ & $-2(1)$ & $-2(1)$ \\
\hline $\mathrm{C}(11)$ & $22(2)$ & $36(2)$ & $60(2)$ & $0(2)$ & $12(2)$ & $-7(1)$ \\
\hline $\mathrm{C}(12)$ & $33(2)$ & $26(2)$ & $50(2)$ & $9(1)$ & $11(2)$ & $-3(1)$ \\
\hline$C(13)$ & $25(2)$ & $22(2)$ & $33(2)$ & $2(1)$ & $5(1)$ & $2(1)$ \\
\hline $\mathrm{C}(14)$ & $24(2)$ & $22(2)$ & $17(1)$ & $-9(1)$ & $-2(1)$ & $5(1)$ \\
\hline$C(15)$ & $31(2)$ & $26(2)$ & $23(2)$ & $-5(1)$ & $-5(1)$ & 2(1) \\
\hline$C(16)$ & $42(2)$ & $26(2)$ & $34(2)$ & $-7(1)$ & $2(1)$ & $-6(1)$ \\
\hline$C(17)$ & $27(2)$ & $31(2)$ & $42(2)$ & $-15(1)$ & $-6(1)$ & $0(1)$ \\
\hline $\mathrm{C}(18)$ & $30(2)$ & $34(2)$ & $30(2)$ & $-12(1)$ & $-12(1)$ & $10(1)$ \\
\hline$C(19)$ & $31(2)$ & $25(2)$ & $22(2)$ & $-6(1)$ & $-3(1)$ & $3(1)$ \\
\hline $\mathrm{C}(20)$ & $19(1)$ & $21(2)$ & $26(2)$ & $-2(1)$ & $3(1)$ & $-6(1)$ \\
\hline $\mathrm{C}(21)$ & $17(1)$ & $20(1)$ & $34(2)$ & $2(1)$ & $5(1)$ & $-3(1)$ \\
\hline $\mathrm{C}(22)$ & $16(1)$ & $26(2)$ & $26(2)$ & $6(1)$ & $4(1)$ & $-4(1)$ \\
\hline $\mathrm{C}(23)$ & $19(2)$ & $26(2)$ & $22(1)$ & 1(1) & $6(1)$ & $-5(1)$ \\
\hline$C(24)$ & $18(1)$ & $23(2)$ & $26(2)$ & $0(1)$ & $5(1)$ & $-4(1)$ \\
\hline$C(25)$ & $32(2)$ & $32(2)$ & $27(2)$ & $-8(1)$ & 2(1) & $-7(1)$ \\
\hline$C(26)$ & $23(2)$ & $24(2)$ & $44(2)$ & $0(1)$ & $8(1)$ & $0(1)$ \\
\hline $\mathrm{C}(27)$ & $28(2)$ & $38(2)$ & $31(2)$ & $13(1)$ & $2(1)$ & $-5(1)$ \\
\hline $\mathrm{C}(28)$ & $26(2)$ & $44(2)$ & $28(2)$ & $-4(1)$ & $12(1)$ & $-2(1)$ \\
\hline $\mathrm{C}(29)$ & $16(2)$ & $30(2)$ & $36(2)$ & $2(1)$ & $0(1)$ & $0(1)$ \\
\hline $\mathrm{C}(30)$ & $18(1)$ & $22(2)$ & $25(2)$ & $0(1)$ & $1(1)$ & $-2(1)$ \\
\hline $\mathrm{C}(31)$ & $23(2)$ & $33(2)$ & $25(2)$ & $-6(1)$ & $-1(1)$ & $4(1)$ \\
\hline $\mathrm{C}(32)$ & $25(2)$ & $40(2)$ & $35(2)$ & $-15(1)$ & $1(1)$ & $4(1)$ \\
\hline $\mathrm{C}(33)$ & $22(2)$ & $20(2)$ & $47(2)$ & $-7(1)$ & $7(1)$ & 1(1) \\
\hline $\mathrm{C}(34)$ & $23(2)$ & $24(2)$ & $37(2)$ & $11(1)$ & $3(1)$ & 1(1) \\
\hline$C(35)$ & $21(2)$ & $27(2)$ & $25(2)$ & $5(1)$ & $4(1)$ & $-1(1)$ \\
\hline$C(36)$ & $22(2)$ & $20(1)$ & $15(1)$ & $3(1)$ & $-1(1)$ & $6(1)$ \\
\hline $\mathrm{C}(37)$ & $26(2)$ & $24(2)$ & $17(1)$ & 1(1) & $-1(1)$ & $6(1)$ \\
\hline $\mathrm{C}(38)$ & $28(2)$ & $20(2)$ & $26(2)$ & $0(1)$ & $-4(1)$ & 2(1) \\
\hline
\end{tabular}




\begin{tabular}{ccccccc}
$\mathrm{C}(39)$ & $27(2)$ & $18(2)$ & $30(2)$ & $6(1)$ & $2(1)$ & $0(1)$ \\
$\mathrm{C}(40)$ & $36(2)$ & $24(2)$ & $22(2)$ & $3(1)$ & $8(1)$ & $4(1)$ \\
$\mathrm{C}(41)$ & $29(2)$ & $18(1)$ & $19(1)$ & $-1(1)$ & $2(1)$ & $2(1)$ \\
$\mathrm{C} 11$ & $33(2)$ & $53(2)$ & $80(3)$ & $-23(2)$ & $-4(2)$ & $-2(2)$ \\
$\mathrm{C} 41$ & $41(2)$ & $50(2)$ & $44(2)$ & $0(2)$ & $6(2)$ & $1(2)$ \\
$\mathrm{C} 51$ & $44(2)$ & $98(3)$ & $46(2)$ & $-7(2)$ & $-12(2)$ & $12(2)$ \\
$\mathrm{C} 61$ & $48(3)$ & $134(5)$ & $72(3)$ & $-63(3)$ & $-17(2)$ & $13(3)$ \\
$\mathrm{C} 21$ & $33(2)$ & $59(2)$ & $43(2)$ & $8(2)$ & $-2(2)$ & $-4(2)$ \\
$\mathrm{C} 31$ & $40(2)$ & $62(2)$ & $33(2)$ & $-7(2)$ & $2(2)$ & $-12(2)$ \\
$\mathrm{C} 12$ & $37(2)$ & $40(2)$ & $49(2)$ & $10(2)$ & $14(2)$ & $10(2)$ \\
$\mathrm{C} 22$ & $32(2)$ & $51(2)$ & $34(2)$ & $-4(2)$ & $0(1)$ & $-5(2)$ \\
$\mathrm{C} 32$ & $27(2)$ & $66(3)$ & $34(2)$ & $14(2)$ & $-4(1)$ & $7(2)$ \\
\hline
\end{tabular}




\section{Computational studies}

\subsection{Thermodynamics of different isomers}

Table S10. Calculated energies [in $\mathrm{kJ} / \mathrm{mol}$ ] of the optimized structures [M062x//6-311+g(d)/LANL2TZ(f)]; energy differences, reaction enthalpies and activation energies are given relative to the energetically most favoured isomer.

\begin{tabular}{|c|c|c|c|c|c|}
\hline Compound & SCF & Enthalpy $^{\mathrm{a}}$ & Free energy $^{a}$ & $\Delta \mathbf{H}$ & $\Delta \mathbf{G}$ \\
\hline \multicolumn{6}{|c|}{ Iridium complex $+\mathrm{Ph}_{2} \mathrm{P}(\mathrm{O}) \mathrm{H}$} \\
\hline cis-isomer (cis-5) & $-8916469,36$ & $-8914433,13$ & $-8914764,78$ & 0 & 0 \\
\hline $\begin{array}{c}\text { cis-isomer (H-bond) } \\
(\text { cis-5') }\end{array}$ & $-8916458,34$ & $-8914421,11$ & $-8914755,52$ & 12,02 & 9,25 \\
\hline $\begin{array}{c}\text { trans-isomer } \\
(\text { trans-5) }\end{array}$ & $-8916437,04$ & $-8914401,58$ & $-8914737,14$ & 31,57 & 27,65 \\
\hline \multicolumn{6}{|c|}{ Iridium complex $+\mathrm{Tol}_{2} \mathrm{P}(\mathrm{O}) \mathrm{H}$} \\
\hline \multirow{2}{*}{$\begin{array}{c}\text { cis-isomer }(\text { cis-5a) } \\
\text { cis-isomer (H-bond) } \\
(\text { cis-5a') }\end{array}$} & $-9122847,25$ & $-9120655,13$ & $-9121005,98$ & 0 & 0 \\
\hline & $-9122838,16$ & $-9120645,46$ & $-9120991,62$ & 9,67 & 14,36 \\
\hline \multicolumn{6}{|c|}{ Ruthenium complex $+\mathrm{Ph}_{2} \mathrm{P}(\mathrm{O}) \mathrm{H}$} \\
\hline cis-isomer (cis-4) & $-8680068,05$ & $-8678219,17$ & $-8678540,46$ & 3,95 & 3,60 \\
\hline $\begin{array}{c}\text { cis-isomer (H-bond) } \\
(\text { cis-4') }\end{array}$ & $-8680072,281$ & $-8678223,12$ & $-8678544,06$ & 0 & 0 \\
\hline
\end{tabular}

[a] Thermal corrections were calculated using the 6-31+G(d) (for all non-metal atoms) and LANL2DZ (for Ir and $\mathrm{Ru}$ ) basis set.
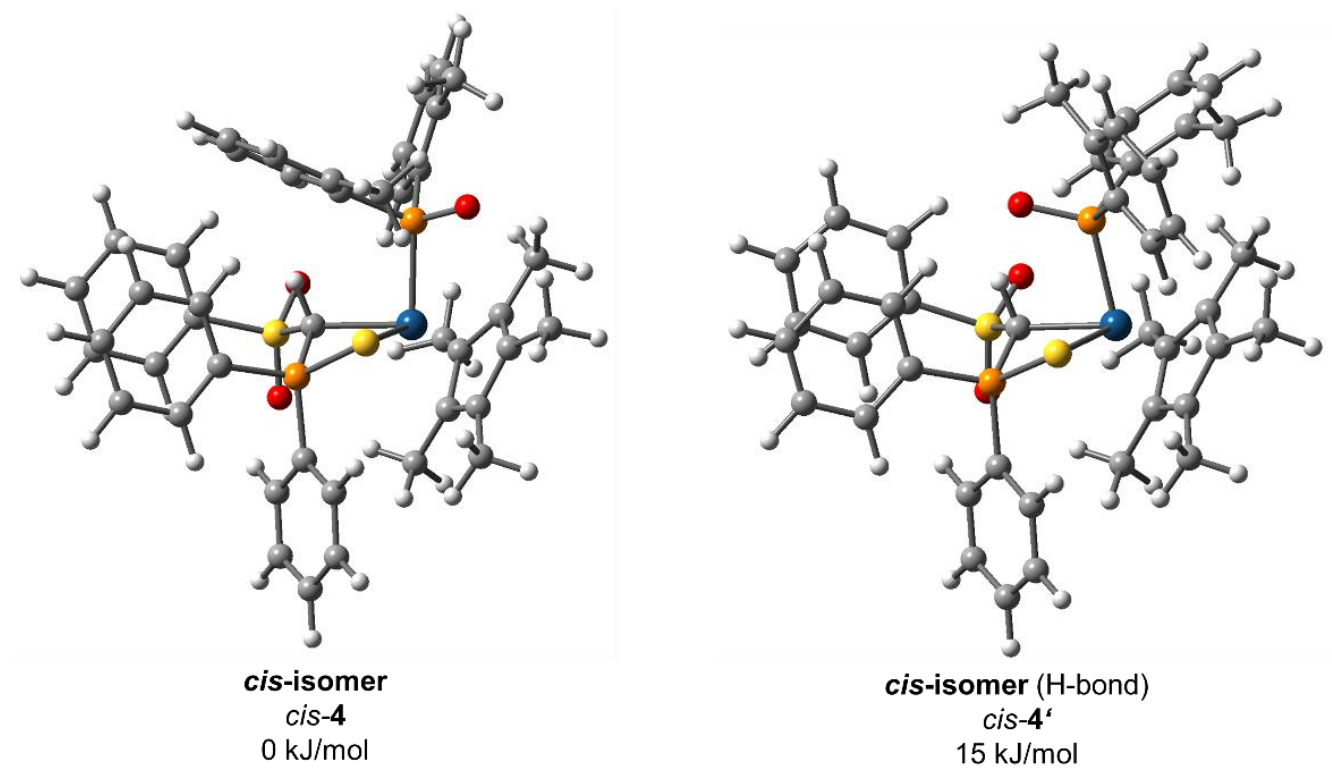

Figure S22. Structures of the energy-optimized cis-isomers of 5b [M062X//6-311+G(d)/LANL2TZ(f)]. 

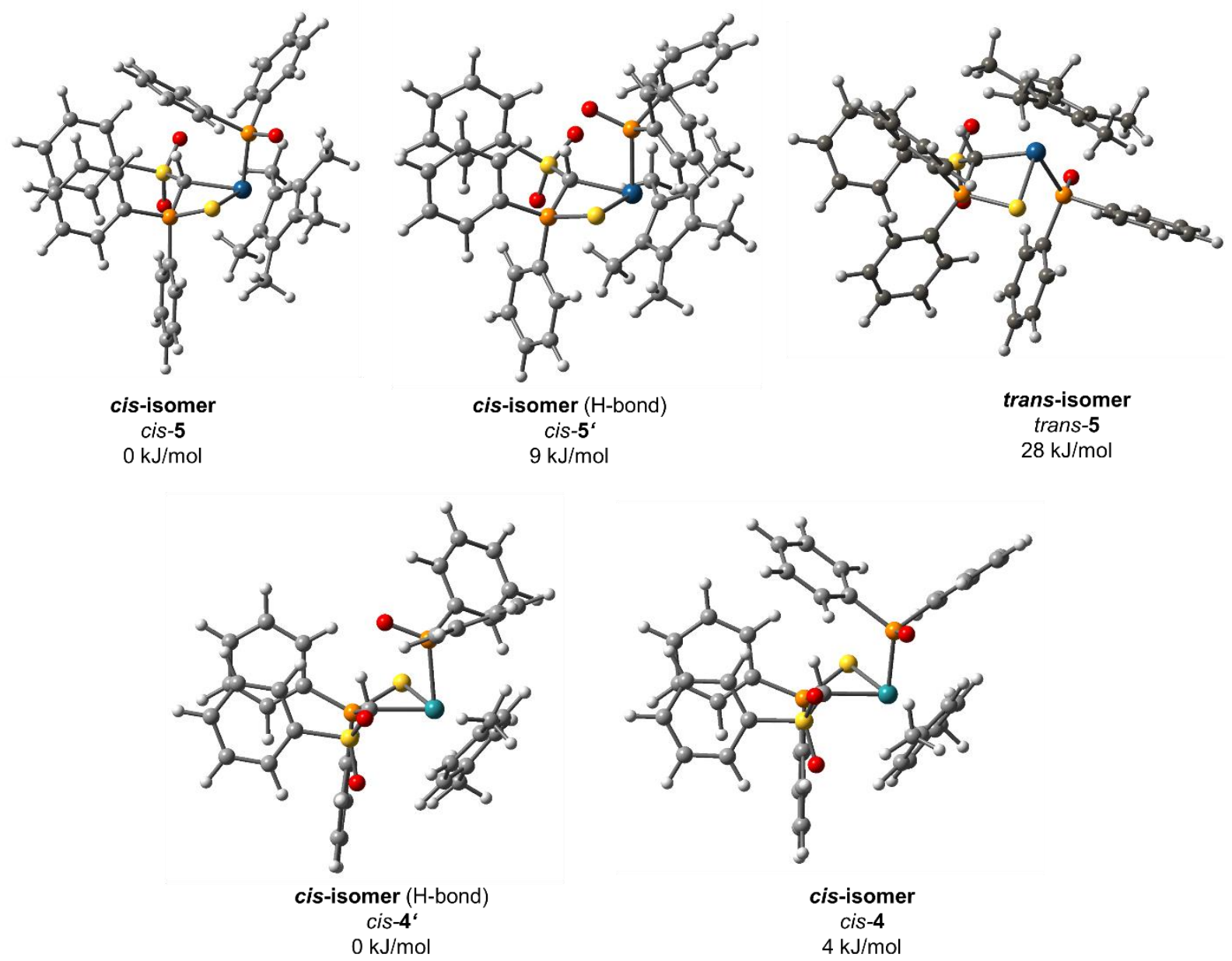

Figure S23. Structures of the energy-optimized isomers of the iridium and ruthenium complexes of the P-H activation of $\mathrm{Ph}_{2} \mathrm{P}(\mathrm{O}) \mathrm{H}[\mathrm{M} 062 \mathrm{X} / / 6-311+\mathrm{G}(\mathrm{d}) / \mathrm{LANL} 2 \mathrm{TZ}(\mathrm{f})]$.

Table S11. Cartesian coordinates of the $c i s$-isomer cis-5.

\begin{tabular}{cccc}
\hline Atomic symbol & $\mathrm{X}$ & $\mathrm{y}$ & $\mathrm{Z}$ \\
\hline $\mathrm{Ir}$ & -1.19375600 & -0.92292700 & -0.22032600 \\
$\mathrm{~S}$ & 0.33015100 & -0.78619300 & -2.18328100 \\
$\mathrm{P}$ & 1.68161000 & -0.64902200 & -0.66976800 \\
$\mathrm{O}$ & 1.27142700 & -0.74810000 & 2.92225700 \\
$\mathrm{C}$ & 0.54035800 & 0.13675600 & 0.48880500 \\
$\mathrm{~S}$ & 0.89257800 & 0.46626000 & 2.19727300 \\
$\mathrm{P}$ & -2.13303800 & 0.97185200 & -1.10652400 \\
$\mathrm{O}$ & -0.21703600 & 1.26696300 & 2.70883400 \\
$\mathrm{C}$ & 2.34666400 & -2.29475300 & -0.27078000 \\
$\mathrm{O}$ & -2.83608400 & 0.68826900 & -2.42001000 \\
$\mathrm{C}$ & 2.59554200 & -2.74961600 & 1.02296300 \\
$\mathrm{C}$ & 3.17025200 & -4.00591000 & 1.21092000 \\
$\mathrm{C}$ & 3.51079400 & -4.79634900 & 0.12000500 \\
$\mathrm{C}$ & 3.27416600 & -4.33501000 & -1.17343000 \\
$\mathrm{C}$ & 2.68916900 & -3.09180700 & -1.36969100 \\
$\mathrm{C}$ & 3.13402200 & 0.37660100 & -1.05802400 \\
$\mathrm{C}$ & 2.90230900 & 1.73040900 & -1.32200300 \\
$\mathrm{C}$ & 3.97065700 & 2.57463600 & -1.58960300 \\
$\mathrm{C}$ & 5.26985000 & 2.07175700 & -1.60867800 \\
$\mathrm{C}$ & 5.50068400 & 0.72369200 & -1.35700500 \\
$\mathrm{C}$ & 4.43566500 & -0.12699400 & -1.07559500
\end{tabular}




\begin{tabular}{|c|c|c|c|}
\hline $\mathrm{C}$ & 2.32608300 & 1.53494900 & 2.14359000 \\
\hline $\mathrm{C}$ & 2.13048400 & 2.90791700 & 2.05845800 \\
\hline $\mathrm{C}$ & 3.23875100 & 3.73528300 & 1.91712400 \\
\hline $\mathrm{C}$ & 4.51610700 & 3.18529800 & 1.86089100 \\
\hline $\mathrm{C}$ & 4.69654600 & 1.80825700 & 1.95895700 \\
\hline $\mathrm{C}$ & 3.59595400 & 0.97179200 & 2.10346300 \\
\hline $\mathrm{C}$ & -2.41272700 & -1.80090900 & 1.34431400 \\
\hline $\mathrm{C}$ & -1.35682300 & -2.78150400 & 1.13378500 \\
\hline $\mathrm{C}$ & -1.40227800 & -3.19761800 & -0.20911200 \\
\hline $\mathrm{C}$ & -2.51050500 & -2.51294600 & -0.86432800 \\
\hline $\mathrm{C}$ & -3.18757900 & -1.73834000 & 0.12783200 \\
\hline $\mathrm{C}$ & -2.78756300 & -1.26204600 & 2.69008300 \\
\hline $\mathrm{C}$ & -0.50528000 & -3.32604600 & 2.23556800 \\
\hline $\mathrm{C}$ & -0.51028100 & -4.18693600 & -0.88857400 \\
\hline $\mathrm{C}$ & -2.96491800 & -2.70145000 & -2.27853300 \\
\hline $\mathrm{C}$ & -4.52767400 & -1.10278400 & -0.06646800 \\
\hline $\mathrm{C}$ & -3.32206800 & 1.78573600 & 0.03585800 \\
\hline $\mathrm{C}$ & -4.48528300 & 2.31350300 & -0.52706300 \\
\hline $\mathrm{C}$ & -5.43693500 & 2.92560800 & 0.28201000 \\
\hline $\mathrm{C}$ & -5.22925900 & 3.01780100 & 1.65647500 \\
\hline $\mathrm{C}$ & -4.06611500 & 2.49924700 & 2.21873400 \\
\hline $\mathrm{C}$ & -3.11494000 & 1.87940400 & 1.41125200 \\
\hline $\mathrm{C}$ & -0.96421900 & 2.38217400 & -1.39511100 \\
\hline $\mathrm{C}$ & -0.55350100 & 2.61314000 & -2.70950800 \\
\hline $\mathrm{C}$ & 0.28777100 & 3.68177600 & -3.00841200 \\
\hline $\mathrm{C}$ & 0.72412700 & 4.53307400 & -1.99611500 \\
\hline $\mathrm{C}$ & 0.31728900 & 4.31113700 & -0.68291900 \\
\hline $\mathrm{C}$ & -0.52573200 & 3.24259900 & -0.38438300 \\
\hline $\mathrm{H}$ & 0.48094900 & 1.15357300 & 0.09162400 \\
\hline $\mathrm{H}$ & 2.31409000 & -2.15124500 & 1.87994400 \\
\hline $\mathrm{H}$ & 3.34426100 & -4.36430000 & 2.21935800 \\
\hline $\mathrm{H}$ & 3.95776100 & -5.77207500 & 0.27445800 \\
\hline $\mathrm{H}$ & 3.53386100 & -4.94819100 & -2.02868000 \\
\hline $\mathrm{H}$ & 2.48039300 & -2.74496600 & -2.37654700 \\
\hline $\mathrm{H}$ & 1.89228600 & 2.12917700 & -1.32856300 \\
\hline $\mathrm{H}$ & 3.78133700 & 3.62436700 & -1.78486800 \\
\hline $\mathrm{H}$ & 6.10319400 & 2.73225500 & -1.82199200 \\
\hline $\mathrm{H}$ & 6.51126900 & 0.33124000 & -1.37510200 \\
\hline $\mathrm{H}$ & 4.62403700 & -1.17388700 & -0.86467900 \\
\hline $\mathrm{H}$ & 1.12612200 & 3.31027500 & 2.11795400 \\
\hline $\mathrm{H}$ & 3.10467800 & 4.80910800 & 1.85221900 \\
\hline $\mathrm{H}$ & 5.37756400 & 3.83311700 & 1.74247100 \\
\hline $\mathrm{H}$ & 5.69329500 & 1.38477400 & 1.91731400 \\
\hline $\mathrm{H}$ & 3.71908100 & -0.10319400 & 2.18723300 \\
\hline $\mathrm{H}$ & -1.91977500 & -0.84122100 & 3.20238700 \\
\hline $\mathrm{H}$ & -3.19746900 & -2.06288500 & 3.31449200 \\
\hline $\mathrm{H}$ & -3.53906700 & -0.47779600 & 2.60878500 \\
\hline $\mathrm{H}$ & -0.07798000 & -2.53457400 & 2.85030800 \\
\hline $\mathrm{H}$ & 0.30768700 & -3.94182900 & 1.85340200 \\
\hline $\mathrm{H}$ & -1.12771800 & -3.95575700 & 2.88032900 \\
\hline $\mathrm{H}$ & -0.11702600 & -3.78063200 & -1.82341000 \\
\hline $\mathrm{H}$ & -1.07334300 & -5.09377500 & -1.12890500 \\
\hline $\mathrm{H}$ & 0.33861700 & -4.46521600 & -0.26259200 \\
\hline $\mathrm{H}$ & -3.30354700 & -1.74901500 & -2.69236000 \\
\hline $\mathrm{H}$ & -3.78090500 & -3.42912700 & -2.33203800 \\
\hline $\mathrm{H}$ & -2.14653600 & -3.06433700 & -2.90293100 \\
\hline $\mathrm{H}$ & -4.75337300 & -0.36870900 & 0.70569000 \\
\hline $\mathrm{H}$ & -5.28659800 & -1.89038700 & -0.02249000 \\
\hline $\mathrm{H}$ & -4.59862100 & -0.61342900 & -1.03956800 \\
\hline $\mathrm{H}$ & -6.34380400 & 3.32670700 & -0.15782600 \\
\hline $\mathrm{H}$ & -5.97331800 & 3.49176700 & 2.28771600 \\
\hline $\mathrm{H}$ & -3.90004700 & 2.56785700 & 3.28878100 \\
\hline
\end{tabular}




\begin{tabular}{cccc}
$\mathrm{H}$ & -2.21965000 & 1.45762300 & 1.85650900 \\
$\mathrm{H}$ & 0.59545900 & 3.85556200 & -4.03388100 \\
$\mathrm{H}$ & 1.36731100 & 5.37475200 & -2.23117700 \\
$\mathrm{H}$ & 0.64270400 & 4.98065400 & 0.10672100 \\
$\mathrm{H}$ & -0.85588100 & 3.08767900 & 0.63997500 \\
$\mathrm{H}$ & -4.63755900 & 2.21576300 & -1.59732600 \\
$\mathrm{H}$ & -0.92057900 & 1.95593200 & -3.49034400 \\
\hline
\end{tabular}

Table S12. Cartesian coordinates of the cis-isomer $c i s-5 '$ '.

\begin{tabular}{|c|c|c|c|}
\hline Atomic symbol & $\mathrm{x}$ & $\mathrm{y}$ & $\mathrm{z}$ \\
\hline $\mathrm{Ir}$ & -0.88034900 & 0.90506000 & -0.07093600 \\
\hline $\mathrm{S}$ & 0.25341700 & 0.66214900 & 2.11082700 \\
\hline $\mathrm{P}$ & 1.86103700 & 0.34990900 & 0.88796000 \\
\hline $\mathrm{O}$ & 2.37464000 & 0.45557000 & -2.60103300 \\
\hline $\mathrm{C}$ & 0.86561000 & -0.24668200 & -0.47791300 \\
\hline $\mathrm{S}$ & 1.50696700 & -0.61009600 & -2.08806300 \\
\hline $\mathrm{P}$ & -1.77198000 & -1.19054700 & 0.38005400 \\
\hline $\mathrm{O}$ & 0.38835000 & -1.05480900 & -2.91256000 \\
\hline $\mathrm{C}$ & 2.81628200 & 1.87364100 & 0.61915100 \\
\hline $\mathrm{O}$ & -0.77201600 & -2.35036300 & 0.37426400 \\
\hline $\mathrm{C}$ & 3.75776800 & 1.96841700 & -0.40990100 \\
\hline $\mathrm{C}$ & 4.49491200 & 3.13579100 & -0.56513600 \\
\hline $\mathrm{C}$ & 4.31064300 & 4.20363200 & 0.30979600 \\
\hline $\mathrm{C}$ & 3.39196600 & 4.10085000 & 1.34843500 \\
\hline $\mathrm{C}$ & 2.64454500 & 2.93728000 & 1.50451300 \\
\hline $\mathrm{C}$ & 3.02801700 & -0.89775100 & 1.49588800 \\
\hline $\mathrm{C}$ & 2.52507300 & -2.19260500 & 1.67503000 \\
\hline $\mathrm{C}$ & 3.37823500 & -3.19839400 & 2.10803600 \\
\hline $\mathrm{C}$ & 4.71832300 & -2.91985400 & 2.36755300 \\
\hline $\mathrm{C}$ & 5.21240100 & -1.63128100 & 2.19810800 \\
\hline $\mathrm{C}$ & 4.36859400 & -0.61492400 & 1.76071700 \\
\hline $\mathrm{C}$ & 2.52028200 & -2.04944700 & -1.76358200 \\
\hline $\mathrm{C}$ & 1.86772700 & -3.24942200 & -1.49370200 \\
\hline $\mathrm{C}$ & 2.63441800 & -4.36483000 & -1.18149200 \\
\hline $\mathrm{C}$ & 4.02314000 & -4.26701200 & -1.12577400 \\
\hline $\mathrm{C}$ & 4.65686400 & -3.05626400 & -1.38622300 \\
\hline $\mathrm{C}$ & 3.90218200 & -1.93362100 & -1.71446100 \\
\hline $\mathrm{C}$ & -1.35928900 & 1.92702000 & -1.93327900 \\
\hline $\mathrm{C}$ & -0.44153100 & 2.80932900 & -1.22798100 \\
\hline $\mathrm{C}$ & -1.05635700 & 3.17611100 & -0.00961500 \\
\hline $\mathrm{C}$ & -2.35883900 & 2.55407400 & 0.06885200 \\
\hline $\mathrm{C}$ & -2.56047800 & 1.83541200 & -1.15333800 \\
\hline $\mathrm{C}$ & -1.21328300 & 1.47012900 & -3.35062900 \\
\hline $\mathrm{C}$ & 0.85381600 & 3.31812300 & -1.78305600 \\
\hline $\mathrm{C}$ & -0.52511800 & 4.09516800 & 1.04430600 \\
\hline $\mathrm{C}$ & -3.40806000 & 2.89933300 & 1.08011100 \\
\hline $\mathrm{C}$ & -3.87192100 & 1.30846100 & -1.63521100 \\
\hline $\mathrm{C}$ & -2.66502600 & -1.26249100 & 1.98543300 \\
\hline $\mathrm{C}$ & -3.01233400 & -2.53099900 & 2.45956800 \\
\hline $\mathrm{C}$ & -3.71559100 & -2.66720500 & 3.65041900 \\
\hline $\mathrm{C}$ & -4.07247900 & -1.53743900 & 4.38460800 \\
\hline $\mathrm{C}$ & -3.71234300 & -0.27434700 & 3.92922200 \\
\hline $\mathrm{C}$ & -3.00770900 & -0.14121300 & 2.73431300 \\
\hline $\mathrm{C}$ & -3.09376100 & -1.63408700 & -0.81498500 \\
\hline $\mathrm{C}$ & -2.67923400 & -1.92134400 & -2.12033400 \\
\hline $\mathrm{C}$ & -3.61286400 & -2.25168000 & -3.09547900 \\
\hline $\mathrm{C}$ & -4.96984700 & -2.29956900 & -2.77791700 \\
\hline
\end{tabular}




\begin{tabular}{cccc}
$\mathrm{C}$ & -5.38530300 & -2.02999200 & -1.47888200 \\
$\mathrm{C}$ & -4.44977300 & -1.70235800 & -0.49860700 \\
$\mathrm{H}$ & 0.59293900 & -1.26011400 & -0.14262100 \\
$\mathrm{H}$ & 3.88975800 & 1.15393500 & -1.10919200 \\
$\mathrm{H}$ & 5.20947400 & 3.21263000 & -1.37642700 \\
$\mathrm{H}$ & 4.88679000 & 5.11345800 & 0.18306600 \\
$\mathrm{H}$ & 3.25113600 & 4.92633200 & 2.03719400 \\
$\mathrm{H}$ & 1.91877300 & 2.85552300 & 2.30555500 \\
$\mathrm{H}$ & 1.47613700 & -2.41072700 & 1.47857900 \\
$\mathrm{H}$ & 2.99491400 & -4.20402400 & 2.23803600 \\
$\mathrm{H}$ & 5.37879500 & -3.71117400 & 2.70503900 \\
$\mathrm{H}$ & 6.25403300 & -1.41427800 & 2.40596300 \\
$\mathrm{H}$ & 4.75568900 & 0.38905300 & 1.62688500 \\
$\mathrm{H}$ & 0.78265600 & -3.29426000 & -1.49729700 \\
$\mathrm{H}$ & 2.14470800 & -5.30809000 & -0.96852000 \\
$\mathrm{H}$ & 4.61508800 & -5.13929300 & -0.87082200 \\
$\mathrm{H}$ & 5.73726400 & -2.98406700 & -1.33717300 \\
$\mathrm{H}$ & 4.37516500 & -0.98560100 & -1.94388300 \\
$\mathrm{H}$ & -0.16641600 & 1.41036200 & -3.64677200 \\
$\mathrm{H}$ & -1.72011200 & 2.17830200 & -4.01522500 \\
$\mathrm{H}$ & -1.64684500 & 0.48110900 & -3.49590400 \\
$\mathrm{H}$ & 1.44397200 & 2.52057600 & -2.23530800 \\
$\mathrm{H}$ & 1.46536900 & 3.79273600 & -1.01434000 \\
$\mathrm{H}$ & 0.64758000 & 4.06431600 & -2.55722300 \\
$\mathrm{H}$ & -0.49831800 & 3.60397100 & 2.02013100 \\
$\mathrm{H}$ & -1.17576000 & 4.97074600 & 1.12850800 \\
$\mathrm{H}$ & 0.47992500 & 4.44142800 & 0.80918200 \\
$\mathrm{H}$ & -4.15626700 & 2.11174100 & 1.17399500 \\
$\mathrm{H}$ & -3.92492800 & 3.81866400 & 0.78467300 \\
$\mathrm{H}$ & -2.97315900 & 3.07057800 & 2.06709800 \\
$\mathrm{H}$ & -3.75773800 & 0.49527500 & -2.35062800 \\
$\mathrm{H}$ & -4.40164100 & 2.12768000 & -2.13358900 \\
$\mathrm{H}$ & -4.49651400 & 0.95322800 & -0.81703800 \\
$\mathrm{H}$ & -3.98305600 & -3.65463400 & 4.01082700 \\
$\mathrm{H}$ & -4.61974800 & -1.64467300 & 5.31484800 \\
$\mathrm{H}$ & -3.97085300 & 0.60669100 & 4.50707900 \\
$\mathrm{H}$ & -2.70271000 & 0.83886200 & 2.38889000 \\
$\mathrm{H}$ & -3.28218400 & -2.46991100 & -4.10533300 \\
$\mathrm{H}$ & -5.69847600 & -2.55464900 & -3.53983200 \\
$\mathrm{H}$ & -6.43871000 & -2.07681700 & -1.22416500 \\
$\mathrm{H}$ & -4.78541500 & -1.49249500 & 0.51206700 \\
$\mathrm{H}$ & -2.71849100 & -3.40558700 & 1.88797800 \\
$\mathrm{H}$ & -1.62370300 & -1.87061100 & -2.37140300 \\
\hline & & & \\
\hline & & & \\
\hline
\end{tabular}

Table S13. Cartesian coordinates of the trans-isomer trans-5.

\begin{tabular}{cccc}
\hline Atomic symbol & $\mathrm{x}$ & $\mathrm{y}$ & $\mathrm{z}$ \\
\hline $\mathrm{Ir}$ & -1.04606000 & 0.96678500 & 0.14882500 \\
$\mathrm{~S}$ & -0.38227900 & -0.04091100 & -2.04452000 \\
$\mathrm{P}$ & 1.43312000 & -0.08509700 & -1.15024200 \\
$\mathrm{O}$ & 1.12017000 & -1.94205400 & 1.84278700 \\
$\mathrm{C}$ & 0.96538700 & 0.39936000 & 0.53577000 \\
$\mathrm{~S}$ & 1.43091900 & -0.52848600 & 1.98822000 \\
$\mathrm{P}$ & -2.17643300 & -0.95941300 & 0.74992300 \\
$\mathrm{O}$ & 0.94005300 & 0.24268100 & 3.12410500 \\
$\mathrm{C}$ & 2.38103200 & -1.61548700 & -1.36484800 \\
$\mathrm{O}$ & -2.50588200 & -1.04292200 & 2.22638400 \\
$\mathrm{C}$ & 1.81117600 & -2.81674400 & -0.93116500
\end{tabular}




\begin{tabular}{|c|c|c|c|}
\hline $\mathrm{C}$ & 2.54386000 & -3.99389600 & -1.02179500 \\
\hline $\mathrm{C}$ & 3.83380100 & -3.97810300 & -1.54541700 \\
\hline $\mathrm{C}$ & 4.39381000 & -2.78473300 & -1.99337300 \\
\hline $\mathrm{C}$ & 3.67079300 & -1.60092400 & -1.90534800 \\
\hline $\mathrm{C}$ & 2.50816000 & 1.25771900 & -1.75817800 \\
\hline $\mathrm{C}$ & 2.25226700 & 1.86271100 & -2.98615000 \\
\hline $\mathrm{C}$ & 3.07859100 & 2.88781700 & -3.43870200 \\
\hline $\mathrm{C}$ & 4.15461700 & 3.30970600 & -2.66552000 \\
\hline $\mathrm{C}$ & 4.41642300 & 2.69962800 & -1.44034900 \\
\hline $\mathrm{C}$ & 3.59862200 & 1.67198400 & -0.98660500 \\
\hline $\mathrm{C}$ & 3.22265800 & -0.37698800 & 2.02351400 \\
\hline $\mathrm{C}$ & 3.78201000 & 0.77402400 & 2.57269600 \\
\hline $\mathrm{C}$ & 5.15992300 & 0.94832100 & 2.51856600 \\
\hline $\mathrm{C}$ & 5.96144100 & -0.02401300 & 1.92160400 \\
\hline $\mathrm{C}$ & 5.39120700 & -1.18177000 & 1.40108600 \\
\hline $\mathrm{C}$ & 4.01209500 & -1.36860800 & 1.45427800 \\
\hline $\mathrm{C}$ & -1.00053100 & 3.17113200 & -0.31378400 \\
\hline $\mathrm{C}$ & -0.61897800 & 2.97581500 & 1.03573800 \\
\hline $\mathrm{C}$ & -1.68562500 & 2.28497400 & 1.73005100 \\
\hline $\mathrm{C}$ & -2.79016000 & 2.17401700 & 0.79943600 \\
\hline $\mathrm{C}$ & -2.35140900 & 2.65125400 & -0.46744500 \\
\hline $\mathrm{C}$ & -0.21806900 & 3.83402700 & -1.40429600 \\
\hline $\mathrm{C}$ & 0.66146500 & 3.40019300 & 1.68095600 \\
\hline $\mathrm{C}$ & -1.76915100 & 1.96399000 & 3.18911600 \\
\hline $\mathrm{C}$ & -4.16872100 & 1.76348300 & 1.20620900 \\
\hline $\mathrm{C}$ & -3.16033400 & 2.78107300 & -1.72036500 \\
\hline $\mathrm{C}$ & -3.78133000 & -0.97690700 & -0.18616600 \\
\hline $\mathrm{C}$ & -4.89144700 & -1.47839300 & 0.49629600 \\
\hline $\mathrm{C}$ & -6.13980100 & -1.51603800 & -0.11602100 \\
\hline $\mathrm{C}$ & -6.29231500 & -1.05932400 & -1.42302400 \\
\hline $\mathrm{C}$ & -5.18870000 & -0.56921100 & -2.11475900 \\
\hline $\mathrm{C}$ & -3.94145800 & -0.52630300 & -1.49564800 \\
\hline $\mathrm{C}$ & -1.58268300 & -2.62453500 & 0.20861500 \\
\hline $\mathrm{C}$ & -1.13285700 & -3.48785700 & 1.20787000 \\
\hline $\mathrm{C}$ & -0.73429800 & -4.78392200 & 0.89025700 \\
\hline $\mathrm{C}$ & -0.80190500 & -5.23421100 & -0.42512400 \\
\hline $\mathrm{C}$ & -1.27797300 & -4.38742400 & -1.42369400 \\
\hline $\mathrm{C}$ & -1.67141300 & -3.09148500 & -1.10629100 \\
\hline $\mathrm{H}$ & 1.50072000 & 1.33591000 & 0.70785900 \\
\hline $\mathrm{H}$ & 0.81891000 & -2.83326800 & -0.49937700 \\
\hline $\mathrm{H}$ & 2.09775500 & -4.91788300 & -0.67354600 \\
\hline $\mathrm{H}$ & 4.40242900 & -4.89929600 & -1.61103100 \\
\hline $\mathrm{H}$ & 5.39356800 & -2.77341500 & -2.41254300 \\
\hline $\mathrm{H}$ & 4.11643500 & -0.67512800 & -2.24944000 \\
\hline $\mathrm{H}$ & 1.39820000 & 1.54370700 & -3.57355500 \\
\hline $\mathrm{H}$ & 2.87511700 & 3.36076300 & -4.39259700 \\
\hline $\mathrm{H}$ & 4.79208000 & 4.11333700 & -3.01672300 \\
\hline $\mathrm{H}$ & 5.25798100 & 3.02087800 & -0.83701300 \\
\hline $\mathrm{H}$ & 3.81781200 & 1.19157700 & -0.03756400 \\
\hline $\mathrm{H}$ & 3.13942800 & 1.50915600 & 3.04469000 \\
\hline $\mathrm{H}$ & 5.60933300 & 1.83635400 & 2.94827200 \\
\hline $\mathrm{H}$ & 7.03590100 & 0.11506100 & 1.87855100 \\
\hline $\mathrm{H}$ & 6.01662200 & -1.94632100 & 0.95447800 \\
\hline $\mathrm{H}$ & 3.55275500 & -2.27200800 & 1.06859900 \\
\hline $\mathrm{H}$ & 0.85000000 & 3.85991700 & -1.17975400 \\
\hline $\mathrm{H}$ & -0.55697400 & 4.86375600 & -1.55538700 \\
\hline $\mathrm{H}$ & -0.34038000 & 3.29950100 & -2.34910800 \\
\hline $\mathrm{H}$ & 1.47194800 & 3.51830800 & 0.95753400 \\
\hline $\mathrm{H}$ & 0.96422000 & 2.67824000 & 2.44204400 \\
\hline $\mathrm{H}$ & 0.51649200 & 4.36482400 & 2.17556100 \\
\hline $\mathrm{H}$ & -2.18180100 & 0.96135500 & 3.32261400 \\
\hline $\mathrm{H}$ & -2.40747400 & 2.69312500 & 3.69933000 \\
\hline
\end{tabular}




\begin{tabular}{cccc}
$\mathrm{H}$ & -0.78370600 & 1.97132400 & 3.65195400 \\
$\mathrm{H}$ & -4.79339400 & 1.48875300 & 0.35697900 \\
$\mathrm{H}$ & -4.63716200 & 2.60846900 & 1.72147600 \\
$\mathrm{H}$ & -4.13456000 & 0.92099100 & 1.89916900 \\
$\mathrm{H}$ & -2.60052600 & 2.43117000 & -2.59120000 \\
$\mathrm{H}$ & -3.41934500 & 3.83049300 & -1.89156900 \\
$\mathrm{H}$ & -4.08403900 & 2.20716300 & -1.65936100 \\
$\mathrm{H}$ & -6.99718400 & -1.89782300 & 0.42803100 \\
$\mathrm{H}$ & -7.26594600 & -1.08598700 & -1.90023100 \\
$\mathrm{H}$ & -5.29942800 & -0.21338500 & -3.13408800 \\
$\mathrm{H}$ & -3.09406000 & -0.11903300 & -2.03816300 \\
$\mathrm{H}$ & -0.38037100 & -5.44409600 & 1.67491300 \\
$\mathrm{H}$ & -0.50261400 & -6.24831200 & -0.67076600 \\
$\mathrm{H}$ & -1.35054000 & -4.73786700 & -2.44759100 \\
$\mathrm{H}$ & -2.05746700 & -2.45034300 & -1.88949500 \\
$\mathrm{H}$ & -4.76102000 & -1.81284000 & 1.52092000 \\
$\mathrm{H}$ & -1.09743200 & -3.12933800 & 2.22899500 \\
\hline
\end{tabular}

Table S14. Cartesian coordinates of the cis-isomer cis-5b (o-tolyl-substituted phosphine oxide).

\begin{tabular}{|c|c|c|c|}
\hline Atomic symbol & $\mathrm{X}$ & $\mathrm{y}$ & $\mathrm{Z}$ \\
\hline $\mathrm{Ir}$ & -0.95158100 & -1.19390500 & -0.16394400 \\
\hline $\mathrm{S}$ & 0.45803400 & -0.72822900 & -2.15325500 \\
\hline $\mathrm{P}$ & 1.83428300 & -0.43529000 & -0.68573200 \\
\hline $\mathrm{O}$ & 1.76128700 & -0.77856900 & 2.80629100 \\
\hline $\mathrm{C}$ & 0.62316600 & 0.10393000 & 0.54313600 \\
\hline $\mathrm{S}$ & 1.05113700 & 0.37423000 & 2.24751700 \\
\hline $\mathrm{P}$ & -2.29050100 & 0.55698000 & -0.85086800 \\
\hline $\mathrm{O}$ & -0.14376200 & 0.85648100 & 2.93162900 \\
\hline $\mathrm{C}$ & 2.79219400 & -1.95518400 & -0.36558000 \\
\hline $\mathrm{O}$ & -3.18634100 & 0.12502600 & -1.99639200 \\
\hline $\mathrm{C}$ & 3.48260700 & -2.19246200 & 0.82658000 \\
\hline $\mathrm{C}$ & 4.29010100 & -3.31775300 & 0.94727300 \\
\hline $\mathrm{C}$ & 4.42840000 & -4.20340800 & -0.11724100 \\
\hline $\mathrm{C}$ & 3.75597600 & -3.96088200 & -1.30988400 \\
\hline $\mathrm{C}$ & 2.94049000 & -2.84089800 & -1.43541000 \\
\hline $\mathrm{C}$ & 3.07196300 & 0.83657300 & -1.08094400 \\
\hline $\mathrm{C}$ & 2.59841300 & 2.13562700 & -1.29235400 \\
\hline $\mathrm{C}$ & 3.49429500 & 3.16197500 & -1.55463400 \\
\hline $\mathrm{C}$ & 4.86032600 & 2.89528200 & -1.62193600 \\
\hline $\mathrm{C}$ & 5.33039600 & 1.60042900 & -1.43061100 \\
\hline $\mathrm{C}$ & 4.43911200 & 0.56703400 & -1.15593700 \\
\hline $\mathrm{C}$ & 2.19517500 & 1.75289600 & 2.18506500 \\
\hline $\mathrm{C}$ & 1.67103200 & 3.04039900 & 2.17920700 \\
\hline $\mathrm{C}$ & 2.53736500 & 4.11849900 & 2.03816400 \\
\hline $\mathrm{C}$ & 3.90561600 & 3.90116100 & 1.90596400 \\
\hline $\mathrm{C}$ & 4.41801300 & 2.60715900 & 1.92779600 \\
\hline $\mathrm{C}$ & 3.56058800 & 1.52232100 & 2.07006500 \\
\hline $\mathrm{C}$ & -1.71956800 & -2.40381500 & 1.47011600 \\
\hline $\mathrm{C}$ & -0.60955200 & -3.17927700 & 0.92971600 \\
\hline $\mathrm{C}$ & -0.90283900 & -3.46491600 & -0.41833900 \\
\hline $\mathrm{C}$ & -2.18865100 & -2.88440000 & -0.75532600 \\
\hline $\mathrm{C}$ & -2.72648800 & -2.31131500 & 0.44357100 \\
\hline $\mathrm{C}$ & -1.87790600 & -2.09107800 & 2.92609300 \\
\hline $\mathrm{C}$ & 0.55010100 & -3.67205100 & 1.73748800 \\
\hline $\mathrm{C}$ & -0.08908300 & -4.26564700 & -1.38296400 \\
\hline $\mathrm{C}$ & -2.91405900 & -3.03811700 & -2.05525400 \\
\hline $\mathrm{C}$ & -4.14239400 & -1.84851700 & 0.58625100 \\
\hline $\mathrm{C}$ & -3.33748500 & 1.26192700 & 0.50264900 \\
\hline $\mathrm{C}$ & -4.46658200 & 2.04747300 & 0.19368500 \\
\hline
\end{tabular}




\begin{tabular}{|c|c|c|c|}
\hline $\mathrm{C}$ & -5.23391300 & 2.53858300 & 1.25241500 \\
\hline $\mathrm{C}$ & -4.89802500 & 2.29360200 & 2.58076500 \\
\hline $\mathrm{C}$ & -3.76391200 & 1.54933300 & 2.87668700 \\
\hline $\mathrm{C}$ & -2.99867400 & 1.03590000 & 1.83391500 \\
\hline $\mathrm{C}$ & -1.36758300 & 2.11335400 & -1.31165600 \\
\hline $\mathrm{C}$ & -1.10747100 & 2.47849300 & -2.64825000 \\
\hline $\mathrm{C}$ & -0.43543600 & 3.68340000 & -2.88579900 \\
\hline $\mathrm{C}$ & -0.04116400 & 4.52768400 & -1.85400400 \\
\hline $\mathrm{C}$ & -0.31432000 & 4.17382300 & -0.53839000 \\
\hline $\mathrm{C}$ & -0.97446900 & 2.97646200 & -0.28078100 \\
\hline $\mathrm{H}$ & 0.37992400 & 1.11737400 & 0.21267600 \\
\hline $\mathrm{H}$ & 3.35517700 & -1.53730000 & 1.67578500 \\
\hline $\mathrm{H}$ & 4.80467300 & -3.50473900 & 1.88280900 \\
\hline $\mathrm{H}$ & 5.05863400 & -5.08001000 & -0.01690800 \\
\hline $\mathrm{H}$ & 3.85840300 & -4.64483300 & -2.14476700 \\
\hline $\mathrm{H}$ & 2.40874000 & -2.65954700 & -2.36236300 \\
\hline $\mathrm{H}$ & 1.53394000 & 2.34884500 & -1.26371000 \\
\hline $\mathrm{H}$ & 3.11909200 & 4.16769200 & -1.70825600 \\
\hline $\mathrm{H}$ & 5.55901400 & 3.69839100 & -1.82955100 \\
\hline $\mathrm{H}$ & 6.39250800 & 1.39163300 & -1.49296100 \\
\hline $\mathrm{H}$ & 4.81096800 & -0.43952100 & -0.99829400 \\
\hline $\mathrm{H}$ & 0.60385500 & 3.18532000 & 2.30276800 \\
\hline $\mathrm{H}$ & 2.14395000 & 5.12873000 & 2.03689000 \\
\hline $\mathrm{H}$ & 4.57754700 & 4.74433500 & 1.79050600 \\
\hline $\mathrm{H}$ & 5.48449000 & 2.44079000 & 1.83016800 \\
\hline $\mathrm{H}$ & 3.94988100 & 0.51135500 & 2.09884500 \\
\hline $\mathrm{H}$ & -0.97377900 & -1.64338500 & 3.34150600 \\
\hline $\mathrm{H}$ & -2.08249400 & -3.01507300 & 3.47689900 \\
\hline $\mathrm{H}$ & -2.70507100 & -1.40493700 & 3.10391100 \\
\hline $\mathrm{H}$ & 0.97803800 & -2.88027000 & 2.35343500 \\
\hline $\mathrm{H}$ & 1.34267100 & -4.07070400 & 1.10294700 \\
\hline $\mathrm{H}$ & 0.21592700 & -4.47470100 & 2.40341700 \\
\hline $\mathrm{H}$ & 0.11214700 & -3.69872100 & -2.29470800 \\
\hline $\mathrm{H}$ & -0.64541700 & -5.16375100 & -1.66708300 \\
\hline $\mathrm{H}$ & 0.86303600 & -4.57381700 & -0.95444000 \\
\hline $\mathrm{H}$ & -3.45574600 & -2.12127700 & -2.29295500 \\
\hline $\mathrm{H}$ & -3.61554200 & -3.87764800 & -2.00894900 \\
\hline $\mathrm{H}$ & -2.21241100 & -3.22951500 & -2.86943100 \\
\hline $\mathrm{H}$ & -4.29312100 & -1.23707900 & 1.47403400 \\
\hline $\mathrm{H}$ & -4.78317600 & -2.73274000 & 0.66357900 \\
\hline $\mathrm{H}$ & -4.45866500 & -1.27419100 & -0.28688400 \\
\hline $\mathrm{H}$ & -6.11165400 & 3.13692900 & 1.02680100 \\
\hline $\mathrm{H}$ & -5.51558100 & 2.69593600 & 3.37690100 \\
\hline $\mathrm{H}$ & -3.46747900 & 1.36846400 & 3.90450100 \\
\hline $\mathrm{H}$ & -2.10878200 & 0.46611300 & 2.06136900 \\
\hline $\mathrm{H}$ & -0.23324500 & 3.97032200 & -3.91323700 \\
\hline $\mathrm{H}$ & 0.46138000 & 5.46246100 & -2.08021000 \\
\hline $\mathrm{H}$ & -0.03026300 & 4.82670500 & 0.27992200 \\
\hline $\mathrm{H}$ & -1.21124100 & 2.71496400 & 0.74768600 \\
\hline $\mathrm{C}$ & -1.57550600 & 1.67475300 & -3.83500700 \\
\hline $\mathrm{H}$ & -1.13760300 & 2.07206500 & -4.75241800 \\
\hline $\mathrm{H}$ & -2.66365600 & 1.70915200 & -3.91140800 \\
\hline $\mathrm{H}$ & -1.32008700 & 0.62087100 & -3.74633300 \\
\hline $\mathrm{C}$ & -4.86220200 & 2.39452000 & -1.21971800 \\
\hline $\mathrm{H}$ & -5.69461200 & 3.10013500 & -1.21672000 \\
\hline $\mathrm{H}$ & -5.13707300 & 1.50376200 & -1.78384600 \\
\hline $\mathrm{H}$ & -4.02677900 & 2.84996400 & -1.75914500 \\
\hline
\end{tabular}


Table S15. Cartesian coordinates of the H-bonded cis-isomer cis-5b' (o-tolyl-substituted phosphine oxide).

\begin{tabular}{|c|c|c|c|}
\hline Atomic symbol & $\mathrm{X}$ & $\mathrm{y}$ & $\mathrm{z}$ \\
\hline $\mathrm{Ir}$ & -0.69258800 & 1.04078200 & -0.08666000 \\
\hline $\mathrm{S}$ & 0.37984400 & 0.61653300 & 2.10278300 \\
\hline $\mathrm{P}$ & 1.98561200 & 0.24457500 & 0.89892600 \\
\hline $\mathrm{O}$ & 2.59080800 & 0.48581100 & -2.54704900 \\
\hline $\mathrm{C}$ & 0.97672500 & -0.21873400 & -0.51004800 \\
\hline $\mathrm{S}$ & 1.64521000 & -0.54938800 & -2.11469700 \\
\hline $\mathrm{P}$ & -1.77178000 & -0.99742100 & 0.28536500 \\
\hline $\mathrm{O}$ & 0.53328800 & -0.89186400 & -2.99472400 \\
\hline $\mathrm{C}$ & 3.05163800 & 1.70921400 & 0.73214100 \\
\hline $\mathrm{O}$ & -0.83090700 & -2.20703200 & 0.22548600 \\
\hline $\mathrm{C}$ & 4.02871200 & 1.79413400 & -0.26385600 \\
\hline $\mathrm{C}$ & 4.84727100 & 2.91473700 & -0.33396400 \\
\hline $\mathrm{C}$ & 4.70922000 & 3.94419500 & 0.59373500 \\
\hline $\mathrm{C}$ & 3.75406700 & 3.84944200 & 1.59984800 \\
\hline $\mathrm{C}$ & 2.92521200 & 2.73383900 & 1.67023700 \\
\hline $\mathrm{C}$ & 3.04763900 & -1.10683300 & 1.47743400 \\
\hline $\mathrm{C}$ & 2.45050700 & -2.36830400 & 1.59256800 \\
\hline $\mathrm{C}$ & 3.21839200 & -3.44830700 & 2.00605500 \\
\hline $\mathrm{C}$ & 4.56728200 & -3.27628700 & 2.30927200 \\
\hline $\mathrm{C}$ & 5.15511400 & -2.02090000 & 2.20286300 \\
\hline $\mathrm{C}$ & 4.39708700 & -0.93102800 & 1.78591800 \\
\hline $\mathrm{C}$ & 2.56200500 & -2.06092900 & -1.83004500 \\
\hline $\mathrm{C}$ & 1.83085300 & -3.23038600 & -1.63938100 \\
\hline $\mathrm{C}$ & 2.51740600 & -4.40486900 & -1.36007100 \\
\hline $\mathrm{C}$ & 3.90695100 & -4.39589600 & -1.25771800 \\
\hline $\mathrm{C}$ & 4.62035000 & -3.21558600 & -1.43931000 \\
\hline $\mathrm{C}$ & 3.94593600 & -2.03418400 & -1.73493500 \\
\hline $\mathrm{C}$ & -0.97911700 & 2.19174300 & -1.91714400 \\
\hline $\mathrm{C}$ & -0.06543200 & 2.96973500 & -1.09791300 \\
\hline $\mathrm{C}$ & -0.74800500 & 3.30190100 & 0.09777500 \\
\hline $\mathrm{C}$ & -2.08454800 & 2.75715600 & 0.04818200 \\
\hline $\mathrm{C}$ & -2.23356900 & 2.12135700 & -1.23063100 \\
\hline $\mathrm{C}$ & -0.76095800 & 1.80605700 & -3.34610400 \\
\hline $\mathrm{C}$ & 1.28417300 & 3.44009500 & -1.54744000 \\
\hline $\mathrm{C}$ & -0.23146300 & 4.11643400 & 1.24165500 \\
\hline $\mathrm{C}$ & -3.18296000 & 3.09000000 & 1.01044200 \\
\hline $\mathrm{C}$ & -3.52102300 & 1.70400100 & -1.86045300 \\
\hline $\mathrm{C}$ & -2.64773200 & -1.07631100 & 1.90845300 \\
\hline $\mathrm{C}$ & -3.17687800 & -2.29865900 & 2.36909700 \\
\hline $\mathrm{C}$ & -3.83217400 & -2.31131300 & 3.60235800 \\
\hline $\mathrm{C}$ & -3.97540000 & -1.16097300 & 4.37094600 \\
\hline $\mathrm{C}$ & -3.44468600 & 0.03892700 & 3.91634000 \\
\hline $\mathrm{C}$ & -2.77980100 & 0.06641900 & 2.69552800 \\
\hline $\mathrm{C}$ & -3.07255900 & -1.30676700 & -1.00002800 \\
\hline $\mathrm{C}$ & -2.50024600 & -1.71580000 & -2.21172000 \\
\hline $\mathrm{C}$ & -3.27707000 & -1.98497600 & -3.33043100 \\
\hline $\mathrm{C}$ & -4.65963700 & -1.86506100 & -3.24377300 \\
\hline $\mathrm{C}$ & -5.23568900 & -1.46505800 & -2.04567500 \\
\hline $\mathrm{C}$ & -4.46926700 & -1.16872100 & -0.91214100 \\
\hline $\mathrm{H}$ & 0.61512600 & -1.22097800 & -0.22633100 \\
\hline $\mathrm{H}$ & 4.12568700 & 1.01214500 & -1.00473600 \\
\hline $\mathrm{H}$ & 5.58942300 & 2.98579100 & -1.12067200 \\
\hline $\mathrm{H}$ & 5.34901500 & 4.81755600 & 0.53315600 \\
\hline $\mathrm{H}$ & 3.64836700 & 4.64438500 & 2.32959900 \\
\hline $\mathrm{H}$ & 2.17019800 & 2.66043700 & 2.44465800 \\
\hline $\mathrm{H}$ & 1.39440900 & -2.50154000 & 1.36207700 \\
\hline $\mathrm{H}$ & 2.76191500 & -4.42824100 & 2.08698600 \\
\hline $\mathrm{H}$ & 5.16088200 & -4.12486500 & 2.63151400 \\
\hline $\mathrm{H}$ & 6.20343700 & -1.88676500 & 2.44439500 \\
\hline
\end{tabular}




\begin{tabular}{|c|c|c|c|}
\hline $\mathrm{H}$ & 4.85756500 & 0.04704600 & 1.70253400 \\
\hline $\mathrm{H}$ & 0.74596600 & -3.20892200 & -1.67988000 \\
\hline $\mathrm{H}$ & 1.96511700 & -5.32538400 & -1.20971000 \\
\hline $\mathrm{H}$ & 4.43650400 & -5.31403400 & -1.02788600 \\
\hline $\mathrm{H}$ & 5.70093500 & -3.21257700 & -1.35420300 \\
\hline $\mathrm{H}$ & 4.48291300 & -1.10763200 & -1.90305200 \\
\hline $\mathrm{H}$ & 0.29859100 & 1.69950800 & -3.57718900 \\
\hline $\mathrm{H}$ & -1.17825500 & 2.58286900 & -3.99644300 \\
\hline $\mathrm{H}$ & -1.24102100 & 0.85636000 & -3.58007600 \\
\hline $\mathrm{H}$ & 1.85282100 & 2.63974900 & -2.02227100 \\
\hline $\mathrm{H}$ & 1.87780800 & 3.83084400 & -0.72030100 \\
\hline $\mathrm{H}$ & 1.15998200 & 4.24350900 & -2.28088500 \\
\hline $\mathrm{H}$ & -0.31427100 & 3.57233900 & 2.18563600 \\
\hline $\mathrm{H}$ & -0.81636200 & 5.03652200 & 1.33192000 \\
\hline $\mathrm{H}$ & 0.81253000 & 4.39133500 & 1.09894500 \\
\hline $\mathrm{H}$ & -3.93820900 & 2.30461900 & 1.05366900 \\
\hline $\mathrm{H}$ & -3.68089800 & 4.01797800 & 0.71025700 \\
\hline $\mathrm{H}$ & -2.79772400 & 3.23564200 & 2.02163400 \\
\hline $\mathrm{H}$ & -3.39687700 & 0.86351800 & -2.54260600 \\
\hline $\mathrm{H}$ & -3.90429500 & 2.55376200 & -2.43605000 \\
\hline $\mathrm{H}$ & -4.27366200 & 1.43720600 & -1.12044200 \\
\hline $\mathrm{H}$ & -4.24672700 & -3.24768500 & 3.96347800 \\
\hline $\mathrm{H}$ & -4.49422100 & -1.20702100 & 5.32242700 \\
\hline $\mathrm{H}$ & -3.53860300 & 0.94362600 & 4.50726000 \\
\hline $\mathrm{H}$ & -2.35918200 & 0.99909900 & 2.33925700 \\
\hline $\mathrm{H}$ & -2.80245500 & -2.28510200 & -4.25811500 \\
\hline $\mathrm{H}$ & -5.28735300 & -2.07506900 & -4.10312400 \\
\hline $\mathrm{H}$ & -6.31504700 & -1.36214200 & -1.98422400 \\
\hline $\mathrm{H}$ & -1.42182700 & -1.80300900 & -2.28316000 \\
\hline $\mathrm{C}$ & -3.09806000 & -3.57563000 & 1.57107600 \\
\hline $\mathrm{H}$ & -3.60080900 & -4.38613000 & 2.10086900 \\
\hline $\mathrm{H}$ & -2.06175500 & -3.84768500 & 1.37509600 \\
\hline $\mathrm{H}$ & -3.57203900 & -3.46056400 & 0.59171500 \\
\hline $\mathrm{C}$ & -5.21228400 & -0.68935400 & 0.31362300 \\
\hline $\mathrm{H}$ & -6.21932800 & -0.37278400 & 0.03587900 \\
\hline $\mathrm{H}$ & -4.71516100 & 0.14816500 & 0.80302300 \\
\hline $\mathrm{H}$ & -5.30556700 & -1.47413200 & 1.06819800 \\
\hline
\end{tabular}

Table S16. Cartesian coordinates of the ruthenium complex cis-4.

\begin{tabular}{cccc}
\hline Atomic symbol & $\mathrm{x}$ & $\mathrm{y}$ & $\mathrm{z}$ \\
\hline $\mathrm{Ru}$ & 1.32984500 & -0.99753100 & 0.27084200 \\
$\mathrm{~S}$ & 0.51333800 & -0.25146900 & -2.02747600 \\
$\mathrm{P}$ & -1.21757300 & -0.39798800 & -0.98517000 \\
$\mathrm{O}$ & -0.77152000 & 0.81753600 & 3.08106700 \\
$\mathrm{C}$ & -0.55611400 & 0.01707600 & 0.62913600 \\
$\mathrm{~S}$ & -1.44004700 & -0.09113600 & 2.16062600 \\
$\mathrm{P}$ & 2.36846800 & 1.01270000 & 0.71473000 \\
$\mathrm{O}$ & -1.64989600 & -1.48461400 & 2.57069300 \\
$\mathrm{C}$ & -1.95283600 & -2.04954300 & -1.18670100 \\
$\mathrm{O}$ & 2.72656800 & 1.20732600 & 2.17406800 \\
$\mathrm{C}$ & -2.06994200 & -2.51177000 & -2.50179300 \\
$\mathrm{C}$ & -2.62518100 & -3.75943500 & -2.75377700 \\
$\mathrm{C}$ & -3.05876100 & -4.55543800 & -1.69555700 \\
$\mathrm{C}$ & -2.93813700 & -4.09821000 & -0.38857900 \\
$\mathrm{C}$ & -2.38801100 & -2.84437200 & -0.12732700 \\
$\mathrm{C}$ & -2.47324600 & 0.78887000 & -1.55488200 \\
$\mathrm{C}$ & -2.05653700 & 2.11189700 & -1.73130800
\end{tabular}




\begin{tabular}{|c|c|c|c|}
\hline $\mathrm{C}$ & -2.97737900 & 3.08163200 & -2.10388100 \\
\hline $\mathrm{C}$ & -4.31195300 & 2.73446400 & -2.30358400 \\
\hline $\mathrm{C}$ & -4.72745900 & 1.42020700 & -2.11861200 \\
\hline $\mathrm{C}$ & -3.81132200 & 0.44278500 & -1.74005400 \\
\hline $\mathrm{C}$ & -3.06362500 & 0.57910700 & 1.80850400 \\
\hline $\mathrm{C}$ & -4.17260300 & -0.25557100 & 1.82036300 \\
\hline $\mathrm{C}$ & -5.42217700 & 0.28381200 & 1.52289100 \\
\hline $\mathrm{C}$ & -5.54096900 & 1.63328800 & 1.21157900 \\
\hline $\mathrm{C}$ & -4.41626100 & 2.45732300 & 1.19928100 \\
\hline $\mathrm{C}$ & -3.16759900 & 1.93350300 & 1.50346300 \\
\hline $\mathrm{C}$ & 1.60507800 & -1.58822000 & 3.58161800 \\
\hline $\mathrm{C}$ & 1.82016000 & -2.09930600 & 2.18540900 \\
\hline $\mathrm{C}$ & 0.86121800 & -2.90718900 & 1.50618700 \\
\hline $\mathrm{C}$ & 1.06544500 & -3.35316000 & 0.19414200 \\
\hline $\mathrm{C}$ & 2.23805400 & -2.96792700 & -0.51556500 \\
\hline $\mathrm{C}$ & 2.40522500 & -3.35508500 & -1.95711600 \\
\hline $\mathrm{C}$ & 3.24045400 & -2.24286200 & 0.16001600 \\
\hline $\mathrm{C}$ & 3.01344700 & -1.79379500 & 1.48576400 \\
\hline $\mathrm{C}$ & 3.93591800 & 1.06813900 & -0.26663800 \\
\hline $\mathrm{C}$ & 5.08119200 & 1.48662400 & 0.41201800 \\
\hline $\mathrm{C}$ & 6.31409500 & 1.51570200 & -0.23662600 \\
\hline $\mathrm{C}$ & 6.41262600 & 1.12740700 & -1.56829000 \\
\hline $\mathrm{C}$ & 5.27461000 & 0.70332500 & -2.25167800 \\
\hline $\mathrm{C}$ & 4.04570700 & 0.66671600 & -1.60114800 \\
\hline $\mathrm{C}$ & 1.48513000 & 2.53360400 & 0.14614500 \\
\hline $\mathrm{C}$ & 0.69667800 & 3.18533400 & 1.10097500 \\
\hline $\mathrm{C}$ & -0.00749100 & 4.33845700 & 0.76155000 \\
\hline $\mathrm{C}$ & 0.08680300 & 4.86273200 & -0.52552200 \\
\hline $\mathrm{C}$ & 0.88295000 & 4.22700800 & -1.47466400 \\
\hline $\mathrm{C}$ & 1.57208900 & 3.06257800 & -1.14259700 \\
\hline $\mathrm{H}$ & -0.40489100 & 1.09638100 & 0.53860500 \\
\hline $\mathrm{H}$ & -1.71212600 & -1.90081600 & -3.32465400 \\
\hline $\mathrm{H}$ & -2.71302800 & -4.11326900 & -3.77460000 \\
\hline $\mathrm{H}$ & -3.48605500 & -5.53238300 & -1.89215900 \\
\hline $\mathrm{H}$ & -3.26674400 & -4.71817100 & 0.43799500 \\
\hline $\mathrm{H}$ & -2.27684900 & -2.50857500 & 0.89589500 \\
\hline $\mathrm{H}$ & -1.01431200 & 2.38182600 & -1.58440100 \\
\hline $\mathrm{H}$ & -2.64776100 & 4.10588400 & -2.23926000 \\
\hline $\mathrm{H}$ & -5.03029700 & 3.49116400 & -2.59988200 \\
\hline $\mathrm{H}$ & -5.76833900 & 1.15249000 & -2.26239100 \\
\hline $\mathrm{H}$ & -4.14130300 & -0.57937000 & -1.58695700 \\
\hline $\mathrm{H}$ & -4.05638200 & -1.30073200 & 2.08151400 \\
\hline $\mathrm{H}$ & -6.30040000 & -0.35151000 & 1.53941500 \\
\hline $\mathrm{H}$ & -6.51440900 & 2.04905000 & 0.97557900 \\
\hline $\mathrm{H}$ & -4.51243300 & 3.50743700 & 0.94848000 \\
\hline $\mathrm{H}$ & -2.28224600 & 2.56266800 & 1.50628100 \\
\hline $\mathrm{H}$ & 2.21396200 & -2.16014700 & 4.28778400 \\
\hline $\mathrm{H}$ & 0.55901800 & -1.67872400 & 3.87223500 \\
\hline $\mathrm{H}$ & 1.89598000 & -0.53607400 & 3.63198300 \\
\hline $\mathrm{H}$ & -0.07626400 & -3.11810700 & 2.00679600 \\
\hline $\mathrm{H}$ & 0.29299600 & -3.91816000 & -0.31558100 \\
\hline $\mathrm{H}$ & 3.10763700 & -2.68936700 & -2.46044300 \\
\hline $\mathrm{H}$ & 4.14136200 & -1.94293700 & -0.36213100 \\
\hline $\mathrm{H}$ & 3.74027300 & -1.14767200 & 1.96639300 \\
\hline $\mathrm{H}$ & 4.98701100 & 1.77846800 & 1.45315200 \\
\hline $\mathrm{H}$ & 7.19874000 & 1.84039200 & 0.30061000 \\
\hline $\mathrm{H}$ & 5.34713900 & 0.39556200 & -3.28950300 \\
\hline $\mathrm{H}$ & 3.16956500 & 0.31055700 & -2.13449400 \\
\hline $\mathrm{H}$ & 0.65004600 & 2.78037200 & 2.10848300 \\
\hline $\mathrm{H}$ & -0.61045500 & 4.84114200 & 1.51094600 \\
\hline $\mathrm{H}$ & 0.97070700 & 4.63765100 & -2.47498500 \\
\hline $\mathrm{H}$ & 2.18945400 & 2.57753800 & -1.89036500 \\
\hline
\end{tabular}




\begin{tabular}{cccc}
$\mathrm{H}$ & -0.44527200 & 5.77258200 & -0.78330500 \\
$\mathrm{H}$ & 7.37229100 & 1.15000000 & -2.07312900 \\
$\mathrm{H}$ & 2.78750600 & -4.37712300 & -2.02874500 \\
$\mathrm{H}$ & 1.44974300 & -3.30587600 & -2.48149300 \\
\hline
\end{tabular}

Table S17. Cartesian coordinates of the ruthenium complex cis-4' (H-bond).

\begin{tabular}{|c|c|c|c|}
\hline Atomic symbol & $\mathrm{X}$ & $\mathrm{y}$ & $\mathrm{z}$ \\
\hline $\mathrm{Ru}$ & 0.99931300 & 1.03614700 & -0.35698000 \\
\hline $\mathrm{S}$ & -0.00757800 & 1.02459400 & 1.93879000 \\
\hline $\mathrm{P}$ & -1.59574400 & 0.38828400 & 0.83762200 \\
\hline $\mathrm{O}$ & -0.29094400 & -1.52158400 & -2.77760100 \\
\hline $\mathrm{C}$ & -0.66552400 & -0.29830800 & -0.53188700 \\
\hline $\mathrm{S}$ & -1.31259900 & -0.71828500 & -2.11162800 \\
\hline $\mathrm{P}$ & 2.05840700 & -0.85918200 & 0.48369500 \\
\hline $\mathrm{O}$ & -1.83543200 & 0.45087000 & -2.83256000 \\
\hline $\mathrm{C}$ & -2.71781100 & 1.79359700 & 0.53926100 \\
\hline $\mathrm{O}$ & 1.16596800 & -2.06363300 & 0.77664500 \\
\hline $\mathrm{C}$ & -3.03029700 & 2.56222400 & 1.66701000 \\
\hline $\mathrm{C}$ & -3.87536000 & 3.65717100 & 1.55294500 \\
\hline $\mathrm{C}$ & -4.40435100 & 4.00263800 & 0.31082700 \\
\hline $\mathrm{C}$ & -4.09375700 & 3.24213900 & -0.80957400 \\
\hline $\mathrm{C}$ & -3.25670900 & 2.13198600 & -0.70100300 \\
\hline $\mathrm{C}$ & -2.57472100 & -0.89775200 & 1.66445200 \\
\hline $\mathrm{C}$ & -1.86315500 & -1.97519500 & 2.20629500 \\
\hline $\mathrm{C}$ & -2.56222300 & -3.02488800 & 2.78863200 \\
\hline $\mathrm{C}$ & -3.95526700 & -3.00740600 & 2.82267200 \\
\hline $\mathrm{C}$ & -4.65733800 & -1.94302400 & 2.26762600 \\
\hline $\mathrm{C}$ & -3.96891300 & -0.88209200 & 1.68630700 \\
\hline $\mathrm{C}$ & -2.71234900 & -1.76397800 & -1.72433400 \\
\hline $\mathrm{C}$ & -3.99508400 & -1.33903900 & -2.04133800 \\
\hline $\mathrm{C}$ & -5.07634800 & -2.13193900 & -1.66412500 \\
\hline $\mathrm{C}$ & -4.86052700 & -3.31785500 & -0.97179500 \\
\hline $\mathrm{C}$ & -3.56533800 & -3.72911400 & -0.66008800 \\
\hline $\mathrm{C}$ & -2.47834800 & -2.95406500 & -1.03985000 \\
\hline $\mathrm{C}$ & 3.09424000 & 0.65824600 & -3.15364300 \\
\hline $\mathrm{C}$ & 2.28538500 & 1.51160400 & -2.22145500 \\
\hline $\mathrm{C}$ & 0.93455100 & 1.77192700 & -2.49995600 \\
\hline $\mathrm{C}$ & 0.17894300 & 2.72374300 & -1.75153500 \\
\hline $\mathrm{C}$ & 0.73995500 & 3.35605700 & -0.64365600 \\
\hline $\mathrm{C}$ & -0.05754900 & 4.29470500 & 0.21538900 \\
\hline $\mathrm{C}$ & 2.08931500 & 3.02520500 & -0.28645900 \\
\hline $\mathrm{C}$ & 2.84815100 & 2.13666500 & -1.06655700 \\
\hline $\mathrm{C}$ & 3.39673600 & -1.42966200 & -0.64043600 \\
\hline $\mathrm{C}$ & 3.01178300 & -2.25849700 & -1.69782500 \\
\hline $\mathrm{C}$ & 3.95711200 & -2.71436100 & -2.60984700 \\
\hline $\mathrm{C}$ & 5.29444000 & -2.34751400 & -2.47513300 \\
\hline $\mathrm{C}$ & 5.68565900 & -1.53182000 & -1.41755400 \\
\hline $\mathrm{C}$ & 4.74010700 & -1.07624100 & -0.50089500 \\
\hline $\mathrm{C}$ & 3.00108100 & -0.48454400 & 2.02315800 \\
\hline $\mathrm{C}$ & 3.13340300 & -1.52005000 & 2.94941100 \\
\hline $\mathrm{C}$ & 3.85676300 & -1.32627400 & 4.12204700 \\
\hline $\mathrm{C}$ & 4.45321000 & -0.09566300 & 4.38132300 \\
\hline $\mathrm{C}$ & 4.31821200 & 0.94424900 & 3.46648500 \\
\hline $\mathrm{C}$ & 3.59064200 & 0.74909100 & 2.29592600 \\
\hline $\mathrm{H}$ & -0.33285500 & -1.26859600 & -0.13433400 \\
\hline $\mathrm{H}$ & -2.59650000 & 2.31306900 & 2.63014600 \\
\hline $\mathrm{H}$ & -4.11230000 & 4.24730500 & 2.43077700 \\
\hline $\mathrm{H}$ & -5.05525700 & 4.86505200 & 0.21958100 \\
\hline $\mathrm{H}$ & -4.49871900 & 3.50970000 & -1.77910400 \\
\hline
\end{tabular}




\begin{tabular}{cccc}
$\mathrm{H}$ & -3.00502200 & 1.56023600 & -1.58445700 \\
$\mathrm{H}$ & -0.77577900 & -1.99550200 & 2.14853600 \\
$\mathrm{H}$ & -2.01698500 & -3.86051300 & 3.21263400 \\
$\mathrm{H}$ & -4.49519100 & -3.82998500 & 3.27927500 \\
$\mathrm{H}$ & -5.74150300 & -1.93620000 & 2.28407200 \\
$\mathrm{H}$ & -4.51635900 & -0.05328600 & 1.24936300 \\
$\mathrm{H}$ & -4.13510800 & -0.41160400 & -2.58404200 \\
$\mathrm{H}$ & -6.08482500 & -1.81945200 & -1.91000900 \\
$\mathrm{H}$ & -5.70505700 & -3.92685800 & -0.66868200 \\
$\mathrm{H}$ & -3.40280300 & -4.65008600 & -0.11241500 \\
$\mathrm{H}$ & -1.46328700 & -3.25819000 & -0.80038600 \\
$\mathrm{H}$ & 3.41233000 & 1.26863700 & -4.00424000 \\
$\mathrm{H}$ & 2.49500200 & -0.17012600 & -3.53264000 \\
$\mathrm{H}$ & 3.98398300 & 0.25249800 & -2.67476800 \\
$\mathrm{H}$ & 0.45549000 & 1.25334400 & -3.32168100 \\
$\mathrm{H}$ & -0.86011200 & 2.88948000 & -2.00926000 \\
$\mathrm{H}$ & -0.01872600 & 3.97809400 & 1.26028900 \\
$\mathrm{H}$ & 2.52055400 & 3.47357600 & 0.60158500 \\
$\mathrm{H}$ & 3.87079800 & 1.90330300 & -0.78856900 \\
$\mathrm{H}$ & 1.96628000 & -2.53097900 & -1.80733800 \\
$\mathrm{H}$ & 3.64952500 & -3.35472400 & -3.42953300 \\
$\mathrm{H}$ & 6.72873500 & -1.25849900 & -1.29880000 \\
$\mathrm{H}$ & 5.05775200 & -0.46041400 & 0.33497200 \\
$\mathrm{H}$ & 2.65387300 & -2.47001600 & 2.73837300 \\
$\mathrm{H}$ & 3.95283200 & -2.13619000 & 4.83720000 \\
$\mathrm{H}$ & 4.77246400 & 1.90835400 & 3.66900000 \\
$\mathrm{H}$ & 3.47280000 & 1.56856000 & 1.59497400 \\
$\mathrm{H}$ & 5.01567300 & 0.05521200 & 5.29625800 \\
$\mathrm{H}$ & 6.03101100 & -2.70443700 & -3.18678100 \\
$\mathrm{H}$ & 0.34607900 & 5.30847000 & 0.14814300 \\
\hline & -1.10472300 & 4.31579400 & -0.09215900 \\
\hline
\end{tabular}

\subsection{Mechanistic Studies}

Table S18. Calculated energies [in $\mathrm{kJ} / \mathrm{mol}]$ of the optimized structures and transition states $[\mathrm{M} 062 \mathrm{x} / / 6$ $311+\mathrm{g}(\mathrm{d}) / \mathrm{LANL} 2 \mathrm{TZ}(\mathrm{f})]$; energy differences, reaction enthalpies and activation energies are given relative to the Iridium complex 2 ' and the respective diphenylphosphine oxide.

\begin{tabular}{cccccc}
\hline Compound & SCF & Enthalpy & Free energy & $\Delta \mathbf{H}$ & $\Delta \mathbf{G}$ \\
\hline Iridium complex 2' & -4578874.748 & -4578249.006 & -4578420.734 & 0.00 & 0.00 \\
Diphenyl phosphine oxid & -2311416.030 & -2310862.805 & -2311002.356 & 0.00 & 0.00 \\
Diphenyl phosphinous acid & -2311416.116 & -2310860.369 & -2311003.275 & 0.00 & 0.00 \\
\hline \multicolumn{7}{c}{ Path via Diphenyl phosphine oxid } \\
\hline TS1 & -6890246.648 & -6889069.364 & -6889314.822 & 42.45 & 108.27 \\
Int1 & -6890319.030 & -6889136.617 & -6889381.746 & -24.81 & 41.34 \\
TS2 & -6890308.604 & -6889131.710 & -6889373.983 & -19.90 & 49.11 \\
$5_{\mathrm{Me}}$ & -6890483.160 & -6889293.742 & -6889533.873 & -181.93 & -110.78 \\
\hline \multicolumn{7}{c}{ Path via Diphenyl phosphinous acid } & & \\
\hline TS1' & -6890333.182 & -6889149.103 & -6889402.168 & -39.73 & 21.84 \\
Int1' & -6890435.678 & -6889249.505 & -6889495.328 & -140.13 & -71.32 \\
TS2' & -6890428.399 & -6889252.987 & -6889495.690 & -143.61 & -71.68 \\
5' $^{\prime}$ & -6890498.303 & -6889309.264 & -6889552.766 & -199.89 & -128.76 \\
\hline
\end{tabular}




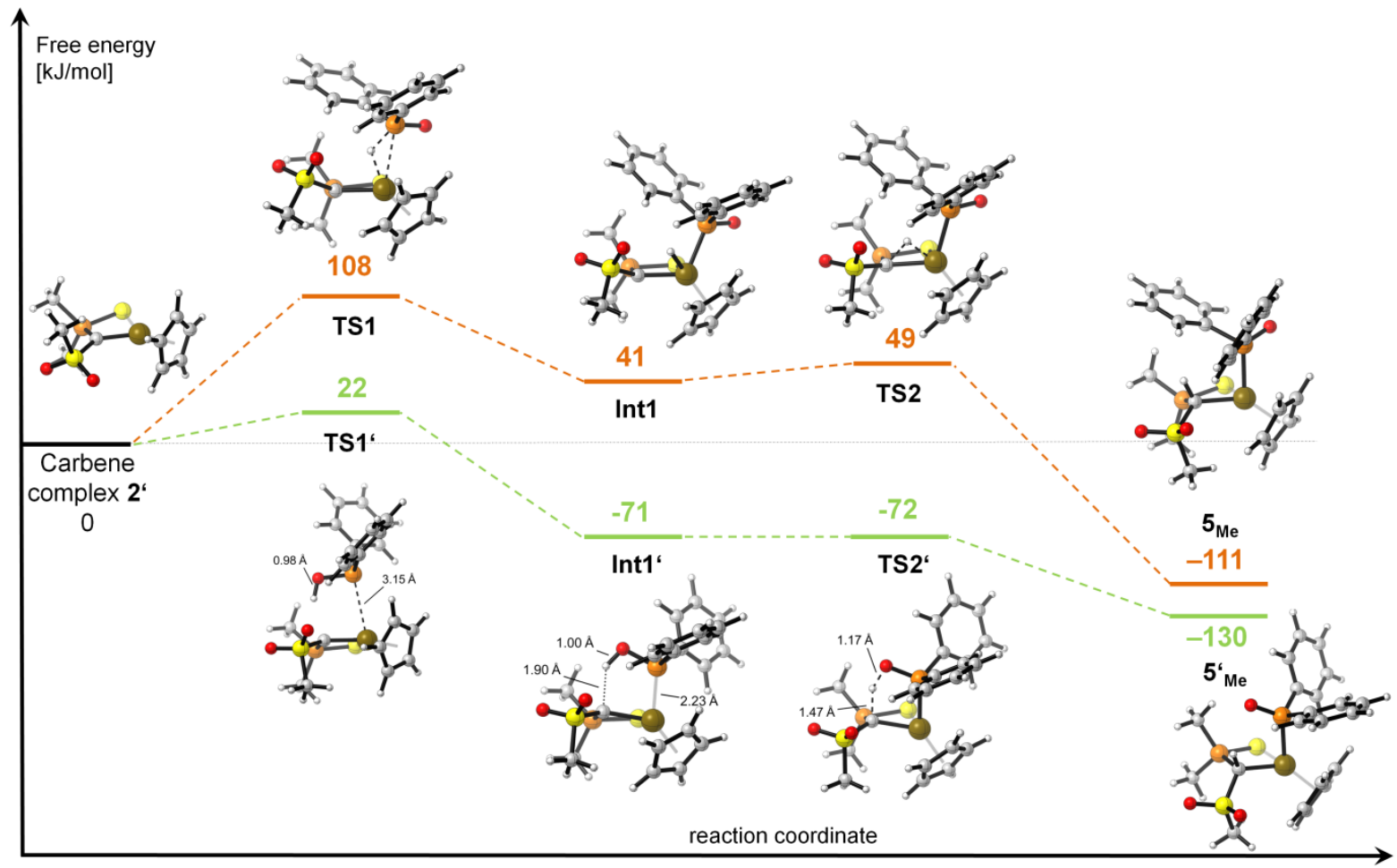

Figure S24. Reaction profile and structures of the optimized intermediates and transition states for the oxidative addition of the $\mathrm{P}-\mathrm{H}$ bond to iridium (orange pathway) and for coordination of the phosphinous acid tautomer (green pathway) [M062X//6-311+G(d)/LANL2TZ(f)].

Table S19. Cartesian Coordinates of the iridium complex 2'.

\begin{tabular}{cccc}
\hline Atomic symbol & $\mathrm{x}$ & $\mathrm{y}$ & $\mathrm{z}$ \\
\hline $\mathrm{Ir}$ & 0.94863900 & -0.17471600 & 0.05247700 \\
$\mathrm{~S}$ & -0.00199300 & -2.44209000 & 0.20658100 \\
$\mathrm{~S}$ & -1.76725800 & 1.68576900 & -0.16186800 \\
$\mathrm{P}$ & -1.72392100 & -1.34466100 & -0.02265600 \\
$\mathrm{O}$ & -3.08394100 & 1.39698000 & -0.75326500 \\
$\mathrm{O}$ & -0.88995000 & 2.67173800 & -0.79659800 \\
$\mathrm{C}$ & -0.92960800 & 0.19294700 & 0.05892300 \\
$\mathrm{C}$ & 3.17463500 & -0.41119800 & 0.06601800 \\
$\mathrm{C}$ & 2.72751500 & 0.47324300 & 1.08469300 \\
$\mathrm{C}$ & 2.04130800 & 1.58461900 & 0.45766900 \\
$\mathrm{C}$ & 2.01760400 & 1.33638000 & -0.94498600 \\
$\mathrm{C}$ & 2.70016500 & 0.08077700 & -1.17944200 \\
$\mathrm{H}$ & 3.67074500 & -1.35612400 & 0.22485900 \\
$\mathrm{H}$ & 2.87933900 & 0.34336200 & 2.14509300 \\
$\mathrm{H}$ & 1.60499600 & 2.43751000 & 0.95361700 \\
$\mathrm{H}$ & 1.52731200 & 1.95495700 & -1.68087300 \\
$\mathrm{H}$ & 2.81871600 & -0.40038900 & -2.13784500 \\
$\mathrm{C}$ & -2.96325200 & -1.66543000 & 1.26380000 \\
$\mathrm{H}$ & -3.79863900 & -0.97501400 & 1.12225200 \\
$\mathrm{H}$ & -3.32554100 & -2.69281100 & 1.19859300 \\
$\mathrm{H}$ & -2.50542400 & -1.50320800 & 2.23937400
\end{tabular}




\begin{tabular}{llll}
$\mathrm{C}$ & -2.56685700 & -1.68519100 & -1.58873500 \\
$\mathrm{H}$ & -2.95777200 & -2.70397400 & -1.59637900 \\
$\mathrm{H}$ & -3.37266300 & -0.95890200 & -1.70859100 \\
$\mathrm{H}$ & -1.84515600 & -1.56140300 & -2.39560900 \\
$\mathrm{C}$ & -2.08905500 & 2.30928900 & 1.48836000 \\
$\mathrm{H}$ & -2.61025600 & 3.25910100 & 1.36976200 \\
$\mathrm{H}$ & -1.13524800 & 2.44842300 & 1.99326200 \\
$\mathrm{H}$ & -2.71236200 & 1.59033100 & 2.01727800 \\
\hline
\end{tabular}

Table S20. Cartesian Coordinates of TS1.

\begin{tabular}{|c|c|c|c|}
\hline Atomic symbol & $\mathrm{x}$ & $\mathrm{y}$ & $\mathrm{z}$ \\
\hline $\mathrm{S}$ & 1.55688100 & 0.15384300 & -2.09507400 \\
\hline $\mathrm{S}$ & 1.54184700 & -0.18732100 & 2.27902500 \\
\hline $\mathrm{P}$ & 2.69572900 & -0.40100200 & -0.45306800 \\
\hline $\mathrm{O}$ & 2.36071600 & -1.40154000 & 2.46857400 \\
\hline $\mathrm{O}$ & 0.12627000 & -0.23495700 & 2.71035700 \\
\hline $\mathrm{C}$ & 0.75194800 & 3.35545600 & -0.55780200 \\
\hline $\mathrm{C}$ & 0.10222700 & 3.12568200 & 0.70776800 \\
\hline $\mathrm{C}$ & -1.21808800 & 2.67197000 & 0.45282500 \\
\hline $\mathrm{C}$ & -1.43181500 & 2.68920800 & -0.97950300 \\
\hline $\mathrm{C}$ & -0.23562600 & 3.11194900 & -1.59110600 \\
\hline $\mathrm{H}$ & 1.76056200 & 3.70738900 & -0.70767800 \\
\hline $\mathrm{H}$ & 0.56499000 & 3.20363700 & 1.67863300 \\
\hline $\mathrm{H}$ & -1.93678500 & 2.35841200 & 1.19518300 \\
\hline $\mathrm{H}$ & -2.32467800 & 2.36029600 & -1.49091200 \\
\hline $\mathrm{H}$ & -0.05315400 & 3.15743000 & -2.65407400 \\
\hline $\mathrm{C}$ & 4.36945300 & 0.30844800 & -0.58567300 \\
\hline $\mathrm{H}$ & 4.93416700 & 0.05075000 & 0.31422900 \\
\hline $\mathrm{H}$ & 4.88944800 & -0.07756100 & -1.46479000 \\
\hline $\mathrm{H}$ & 4.27870700 & 1.39192900 & -0.65909500 \\
\hline $\mathrm{C}$ & 3.00422900 & -2.18917400 & -0.41661100 \\
\hline $\mathrm{H}$ & 3.64759000 & -2.45995200 & -1.25666300 \\
\hline $\mathrm{H}$ & 3.47405200 & -2.44893400 & 0.53239300 \\
\hline $\mathrm{H}$ & 2.05391300 & -2.71345200 & -0.49782400 \\
\hline $\mathrm{C}$ & 1.67036400 & 0.34804100 & 0.69025400 \\
\hline $\mathrm{H}$ & -0.47386400 & -0.16731100 & 0.05539700 \\
\hline Ir & 0.22998800 & 1.31620600 & -0.44056900 \\
\hline $\mathrm{C}$ & 2.28080900 & 1.02096800 & 3.38802000 \\
\hline $\mathrm{H}$ & 2.18348300 & 0.63145900 & 4.40106700 \\
\hline $\mathrm{H}$ & 1.74524600 & 1.96302200 & 3.29001000 \\
\hline $\mathrm{H}$ & 3.32773000 & 1.13616100 & 3.11376700 \\
\hline $\mathrm{P}$ & -1.46485600 & -0.80594200 & -0.96105300 \\
\hline $\mathrm{C}$ & -0.75924000 & -2.43541900 & -0.63679000 \\
\hline $\mathrm{C}$ & -0.42403000 & -2.83713800 & 0.65853400 \\
\hline $\mathrm{C}$ & -0.51628800 & -3.26745800 & -1.73009300 \\
\hline $\mathrm{C}$ & 0.13298800 & -4.09490900 & 0.85875600 \\
\hline $\mathrm{H}$ & -0.56593900 & -2.17258900 & 1.50531300 \\
\hline $\mathrm{C}$ & 0.03992600 & -4.52501000 & -1.51872200 \\
\hline $\mathrm{H}$ & -0.75922000 & -2.91718100 & -2.72736000 \\
\hline $\mathrm{C}$ & 0.35762700 & -4.93819900 & -0.22688800 \\
\hline $\mathrm{H}$ & 0.41129100 & -4.40136600 & 1.86006300 \\
\hline $\mathrm{H}$ & 0.23033100 & -5.17928200 & -2.36188100 \\
\hline
\end{tabular}




$\begin{array}{lccc}\mathrm{H} & 0.79654100 & -5.91690300 & -0.06679000 \\ \mathrm{C} & -2.91671300 & -0.50322800 & 0.07602800 \\ \mathrm{C} & -2.81997200 & -0.30950600 & 1.45650400 \\ \mathrm{C} & -4.15619600 & -0.45909200 & -0.56453000 \\ \mathrm{C} & -3.97919700 & -0.09138600 & 2.19383800 \\ \mathrm{H} & -1.85062300 & -0.30454900 & 1.94991300 \\ \mathrm{C} & -5.30814700 & -0.23594500 & 0.18262400 \\ \mathrm{H} & -4.20500300 & -0.59224400 & -1.64006500 \\ \mathrm{C} & -5.21906000 & -0.05625900 & 1.55978500 \\ \mathrm{H} & -3.91092000 & 0.05640600 & 3.26566400 \\ \mathrm{H} & -6.27311200 & -0.20059300 & -0.31019200 \\ \mathrm{H} & -6.11813200 & 0.11666800 & 2.14104800 \\ \mathrm{O} & -1.72818900 & -0.54146900 & -2.40096400\end{array}$

Table S21. Cartesian Coordinates of Int1.

\begin{tabular}{|c|c|c|c|}
\hline Atomic symbol & $\mathrm{X}$ & $\mathrm{y}$ & $\mathrm{Z}$ \\
\hline $\mathrm{S}$ & 1.37537800 & -0.09184000 & -2.12276400 \\
\hline $\mathrm{S}$ & 1.90118400 & 0.07483600 & 2.22409700 \\
\hline $\mathrm{P}$ & 2.68767100 & -0.50570900 & -0.56717600 \\
\hline $\mathrm{O}$ & 3.00236900 & -0.88653800 & 2.41937000 \\
\hline $\mathrm{O}$ & 0.59276300 & -0.19790500 & 2.85170700 \\
\hline $\mathrm{C}$ & 0.41274100 & 3.15404300 & -1.53060500 \\
\hline $\mathrm{C}$ & 0.68170900 & 3.36414700 & -0.16884400 \\
\hline $\mathrm{C}$ & -0.47123700 & 2.95518900 & 0.57855200 \\
\hline $\mathrm{C}$ & -1.49179900 & 2.57577500 & -0.35976800 \\
\hline $\mathrm{C}$ & -0.92672700 & 2.64817200 & -1.66508800 \\
\hline $\mathrm{H}$ & 1.11644000 & 3.27658400 & -2.34063000 \\
\hline $\mathrm{H}$ & 1.63089500 & 3.66075400 & 0.25132900 \\
\hline $\mathrm{H}$ & -0.59163500 & 2.99827200 & 1.65072400 \\
\hline $\mathrm{H}$ & -2.49737400 & 2.26955300 & -0.11128000 \\
\hline $\mathrm{H}$ & -1.42311700 & 2.37554300 & -2.58431200 \\
\hline $\mathrm{C}$ & 4.35523600 & 0.14932000 & -0.87896700 \\
\hline $\mathrm{H}$ & 4.95765000 & -0.02409900 & 0.01671400 \\
\hline $\mathrm{H}$ & 4.82168900 & -0.34888000 & -1.73088700 \\
\hline $\mathrm{H}$ & 4.28267300 & 1.21962800 & -1.06802500 \\
\hline $\mathrm{C}$ & 2.95475900 & -2.29138000 & -0.40363800 \\
\hline $\mathrm{H}$ & 3.53967800 & -2.64887500 & -1.25413100 \\
\hline $\mathrm{H}$ & 3.48422000 & -2.47731700 & 0.53204200 \\
\hline $\mathrm{H}$ & 1.99159700 & -2.79778300 & -0.38456100 \\
\hline $\mathrm{C}$ & 1.75669500 & 0.37277500 & 0.56399200 \\
\hline $\mathrm{H}$ & -0.31666300 & 0.28684700 & 0.84069500 \\
\hline Ir & 0.08891700 & 1.07290200 & -0.43367300 \\
\hline $\mathrm{C}$ & 2.44990300 & 1.59087400 & 3.01368500 \\
\hline $\mathrm{H}$ & 2.52060000 & 1.39397100 & 4.08298300 \\
\hline $\mathrm{H}$ & 1.71124000 & 2.36697700 & 2.81834800 \\
\hline $\mathrm{H}$ & 3.42015900 & 1.85775100 & 2.59962900 \\
\hline $\mathrm{P}$ & -1.44490300 & -0.58291300 & -0.92323400 \\
\hline $\mathrm{C}$ & -0.76255900 & -2.22228200 & -0.50643700 \\
\hline $\mathrm{C}$ & -0.40518900 & -2.58977200 & 0.79255900 \\
\hline $\mathrm{C}$ & -0.65245300 & -3.14157000 & -1.54975100 \\
\hline $\mathrm{C}$ & 0.04325000 & -3.88204100 & 1.04585600 \\
\hline $\mathrm{H}$ & -0.44926800 & -1.88079700 & 1.61180200 \\
\hline
\end{tabular}




\begin{tabular}{lccc}
$\mathrm{C}$ & -0.19278800 & -4.43018600 & -1.29172000 \\
$\mathrm{H}$ & -0.93354600 & -2.83502000 & -2.55154300 \\
$\mathrm{C}$ & 0.14805300 & -4.80260900 & 0.00584600 \\
$\mathrm{H}$ & 0.32432500 & -4.16085900 & 2.05501800 \\
$\mathrm{H}$ & -0.10672000 & -5.14432600 & -2.10319600 \\
$\mathrm{H}$ & 0.50126400 & -5.80823800 & 0.20635900 \\
$\mathrm{C}$ & -2.86666400 & -0.35783300 & 0.21852400 \\
$\mathrm{C}$ & -2.75836500 & -0.19024000 & 1.60347100 \\
$\mathrm{C}$ & -4.12711500 & -0.35065000 & -0.38050300 \\
$\mathrm{C}$ & -3.90439700 & -0.02734000 & 2.37365200 \\
$\mathrm{H}$ & -1.79140100 & -0.17690100 & 2.09968600 \\
$\mathrm{C}$ & -5.27109200 & -0.18068100 & 0.39575700 \\
$\mathrm{H}$ & -4.19488900 & -0.47425600 & -1.45598800 \\
$\mathrm{C}$ & -5.16108300 & -0.01994800 & 1.77194400 \\
$\mathrm{H}$ & -3.81401900 & 0.09612800 & 3.44706700 \\
$\mathrm{H}$ & -6.24687400 & -0.17435900 & -0.07720600 \\
$\mathrm{H}$ & -6.05103500 & 0.11183400 & 2.37740900 \\
$\mathrm{O}$ & -1.92816500 & -0.53015400 & -2.34251200 \\
\hline
\end{tabular}

Table S22. Cartesian Coordinates of TS2.

\begin{tabular}{cccc}
\hline Atomic symbol & $\mathrm{X}$ & $\mathrm{y}$ & $\mathrm{z}$ \\
\hline $\mathrm{S}$ & -1.43457300 & 0.15423600 & -2.12853900 \\
$\mathrm{~S}$ & -1.87924600 & -0.14905000 & 2.27333800 \\
$\mathrm{P}$ & -2.65799400 & 0.57560500 & -0.51020600 \\
$\mathrm{O}$ & -3.01240300 & 0.76563000 & 2.49459900 \\
$\mathrm{O}$ & -0.59591900 & 0.10066900 & 2.95203500 \\
$\mathrm{C}$ & -0.62441500 & -3.33555200 & -0.14113800 \\
$\mathrm{C}$ & 0.57444300 & -2.89032800 & 0.52191800 \\
$\mathrm{C}$ & 1.52344000 & -2.53856500 & -0.48715400 \\
$\mathrm{C}$ & 0.87265800 & -2.64388900 & -1.75540600 \\
$\mathrm{C}$ & -0.44612800 & -3.17232800 & -1.52106700 \\
$\mathrm{H}$ & -1.54009400 & -3.63523700 & 0.34655200 \\
$\mathrm{H}$ & 0.76226700 & -2.89518800 & 1.58546200 \\
$\mathrm{H}$ & 2.53840500 & -2.21254000 & -0.31449600 \\
$\mathrm{H}$ & 1.31408900 & -2.40986500 & -2.71205000 \\
$\mathrm{H}$ & -1.19892900 & -3.32779300 & -2.27971400 \\
$\mathrm{C}$ & -4.33304800 & -0.10626200 & -0.69068000 \\
$\mathrm{H}$ & -4.86731600 & 0.06276100 & 0.24785800 \\
$\mathrm{H}$ & -4.86781400 & 0.38181900 & -1.50751000 \\
$\mathrm{H}$ & -4.25704300 & -1.17552100 & -0.88505300 \\
$\mathrm{C}$ & -2.92615200 & 2.35601700 & -0.31497200 \\
$\mathrm{H}$ & -3.59167000 & 2.71469300 & -1.10325400 \\
$\mathrm{H}$ & -3.37171900 & 2.52727700 & 0.66673900 \\
$\mathrm{H}$ & -1.97023400 & 2.87267000 & -0.38115000 \\
$\mathrm{C}$ & -1.67193100 & -0.28253900 & 0.59935400 \\
$\mathrm{H}$ & -0.04611300 & -0.03822900 & 0.67443400 \\
$\mathrm{Ir}$ & -0.08907600 & -1.06448700 & -0.53354000 \\
$\mathrm{C}$ & -2.41496100 & -1.73826100 & 2.91332500 \\
$\mathrm{H}$ & -2.54929500 & -1.62599600 & 3.98875200 \\
$\mathrm{H}$ & -1.63832800 & -2.47241600 & 2.70368300 \\
$\mathrm{H}$ & -3.35265900 & -2.00359500 & 2.42856700 \\
$\mathrm{P}$ & 1.45941000 & 0.58917600 & -0.94194600 \\
& & &
\end{tabular}




\begin{tabular}{cccc} 
C & 0.78847400 & 2.23355900 & -0.50265400 \\
$\mathrm{C}$ & 0.46521800 & 2.59663200 & 0.80716200 \\
$\mathrm{C}$ & 0.65641100 & 3.16092100 & -1.53587900 \\
$\mathrm{C}$ & 0.02642800 & 3.88816000 & 1.08033400 \\
$\mathrm{H}$ & 0.54521800 & 1.88833200 & 1.62584600 \\
$\mathrm{C}$ & 0.20786600 & 4.44968000 & -1.25883900 \\
$\mathrm{H}$ & 0.91656300 & 2.86121700 & -2.54542000 \\
$\mathrm{C}$ & -0.10071100 & 4.81534600 & 0.04875800 \\
$\mathrm{H}$ & -0.22294500 & 4.16373100 & 2.09868100 \\
$\mathrm{H}$ & 0.10744800 & 5.17007900 & -2.06311000 \\
$\mathrm{H}$ & -0.44332700 & 5.82151000 & 0.26431900 \\
$\mathrm{C}$ & 2.82397200 & 0.33247400 & 0.25783500 \\
$\mathrm{C}$ & 2.63532900 & 0.05125900 & 1.61522400 \\
$\mathrm{C}$ & 4.11753000 & 0.41502300 & -0.25878700 \\
$\mathrm{C}$ & 3.73749900 & -0.13000300 & 2.44353600 \\
$\mathrm{H}$ & 1.63892300 & -0.03690800 & 2.04009700 \\
$\mathrm{C}$ & 5.21699800 & 0.22558400 & 0.57492500 \\
$\mathrm{H}$ & 4.24677900 & 0.61920000 & -1.31642400 \\
$\mathrm{C}$ & 5.02810400 & -0.04532400 & 1.92539600 \\
$\mathrm{H}$ & 3.58623000 & -0.34030000 & 3.49648600 \\
$\mathrm{H}$ & 6.21985400 & 0.28856200 & 0.16719700 \\
$\mathrm{H}$ & 5.88363400 & -0.19294700 & 2.57526000 \\
$\mathrm{O}$ & 1.99265400 & 0.57818500 & -2.34423700 \\
\hline
\end{tabular}

Table S23. Cartesian Coordinates of $5_{\mathrm{Me}}$.

\begin{tabular}{cccc}
\hline Atomic symbol & $\mathrm{x}$ & $\mathrm{y}$ & $\mathrm{Z}$ \\
\hline $\mathrm{S}$ & 1.48306600 & -0.99749300 & -1.98208400 \\
$\mathrm{~S}$ & 1.79077200 & 0.13093300 & 2.36707700 \\
$\mathrm{P}$ & 2.05195900 & -1.59013500 & -0.12311400 \\
$\mathrm{O}$ & 2.27717000 & -1.06224100 & 3.06792300 \\
$\mathrm{O}$ & 0.81049600 & 0.99462100 & 3.02157100 \\
$\mathrm{C}$ & 1.91248900 & 2.69456400 & -0.95926400 \\
$\mathrm{C}$ & 0.99602800 & 2.87931500 & 0.11949900 \\
$\mathrm{C}$ & -0.33544500 & 2.86224900 & -0.39878900 \\
$\mathrm{C}$ & -0.24891500 & 2.65044100 & -1.82088400 \\
$\mathrm{C}$ & 1.12389100 & 2.51168000 & -2.14331600 \\
$\mathrm{H}$ & 2.99071100 & 2.69995300 & -0.91430900 \\
$\mathrm{H}$ & 1.23593600 & 3.00788600 & 1.16566500 \\
$\mathrm{H}$ & -1.23821400 & 3.00644800 & 0.17404300 \\
$\mathrm{H}$ & -1.07957300 & 2.52939900 & -2.50010900 \\
$\mathrm{H}$ & 1.51154500 & 2.27929300 & -3.12433900 \\
$\mathrm{C}$ & 3.83968300 & -1.38770500 & 0.11883800 \\
$\mathrm{H}$ & 4.11248100 & -1.62194800 & 1.14950500 \\
$\mathrm{H}$ & 4.33702300 & -2.08250900 & -0.56061700 \\
$\mathrm{H}$ & 4.13857200 & -0.37498700 & -0.14844300 \\
$\mathrm{C}$ & 1.71814500 & -3.30816600 & 0.32452900 \\
$\mathrm{H}$ & 2.32647600 & -3.97684600 & -0.28722200 \\
$\mathrm{H}$ & 1.96697400 & -3.43390900 & 1.38154800 \\
$\mathrm{H}$ & 0.66315800 & -3.52937200 & 0.16449500 \\
$\mathrm{C}$ & 1.05748100 & -0.41745300 & 0.82970900 \\
$\mathrm{H}$ & 0.21678800 & -0.98574000 & 1.24159000 \\
$\mathrm{Ir}$ & 0.52477900 & 0.89022900 & -0.72948900
\end{tabular}




\begin{tabular}{llll}
$\mathrm{C}$ & 3.23158500 & 1.15351500 & 2.01167700 \\
$\mathrm{H}$ & 3.20824600 & 1.95549500 & 2.74812200 \\
$\mathrm{H}$ & 3.15906300 & 1.55698400 & 1.00547900 \\
$\mathrm{H}$ & 4.12455500 & 0.54750900 & 2.13569500 \\
$\mathrm{P}$ & -1.53231000 & -0.10962600 & -0.92814100 \\
$\mathrm{C}$ & -1.59137200 & -1.89356200 & -0.44622900 \\
$\mathrm{C}$ & -1.80566400 & -2.35205100 & 0.85652500 \\
$\mathrm{C}$ & -1.49329400 & -2.82327700 & -1.48573100 \\
$\mathrm{C}$ & -1.89526000 & -3.71737900 & 1.12060600 \\
$\mathrm{H}$ & -1.93960700 & -1.64599900 & 1.67086300 \\
$\mathrm{C}$ & -1.58652700 & -4.18639600 & -1.22293400 \\
$\mathrm{H}$ & -1.37043700 & -2.46076400 & -2.50022700 \\
$\mathrm{C}$ & -1.78308300 & -4.63614500 & 0.08133300 \\
$\mathrm{H}$ & -2.06818700 & -4.05936100 & 2.13497600 \\
$\mathrm{H}$ & -1.51814500 & -4.89859000 & -2.03803900 \\
$\mathrm{H}$ & -1.86329200 & -5.69844700 & 0.28461500 \\
$\mathrm{C}$ & -2.68332300 & 0.62456400 & 0.30292800 \\
$\mathrm{C}$ & -2.29819200 & 0.99089100 & 1.59545300 \\
$\mathrm{C}$ & -4.00844000 & 0.79828600 & -0.09723500 \\
$\mathrm{C}$ & -3.23739200 & 1.50477000 & 2.48413600 \\
$\mathrm{H}$ & -1.26255800 & 0.90310500 & 1.91273700 \\
$\mathrm{C}$ & -4.94397100 & 1.32230500 & 0.79122500 \\
$\mathrm{H}$ & -4.28769300 & 0.52848100 & -1.11064300 \\
$\mathrm{C}$ & -4.56110900 & 1.67054400 & 2.08276700 \\
$\mathrm{H}$ & -2.93026100 & 1.78406600 & 3.48604400 \\
$\mathrm{H}$ & -5.97172500 & 1.46051600 & 0.47343200 \\
$\mathrm{H}$ & -5.29048700 & 2.07782000 & 2.77449100 \\
$\mathrm{O}$ & -2.11258600 & 0.01753500 & -2.31559300 \\
\hline & & &
\end{tabular}

Table S24. Cartesian Coordinates of TS1'.

\begin{tabular}{cccc}
\hline Atomic symbol & $\mathrm{x}$ & $\mathrm{y}$ & $\mathrm{z}$ \\
\hline $\mathrm{S}$ & -1.26059300 & -2.20319300 & 1.36402300 \\
$\mathrm{~S}$ & -2.32488700 & 0.46414900 & -2.04711000 \\
$\mathrm{P}$ & -1.94613100 & -2.09609500 & -0.57523400 \\
$\mathrm{O}$ & -2.56779800 & -0.47117300 & -3.15881600 \\
$\mathrm{O}$ & -1.57712600 & 1.70232700 & -2.30005400 \\
$\mathrm{C}$ & -2.41625300 & 1.60265500 & 1.92059300 \\
$\mathrm{C}$ & -1.52314200 & 2.31383600 & 1.03944700 \\
$\mathrm{C}$ & -0.20095400 & 2.09960900 & 1.53041400 \\
$\mathrm{C}$ & -0.26666800 & 1.34725800 & 2.75823000 \\
$\mathrm{C}$ & -1.62380900 & 1.03645300 & 2.99253700 \\
$\mathrm{H}$ & -3.49138800 & 1.54380700 & 1.84078900 \\
$\mathrm{H}$ & -1.77490600 & 2.83617600 & 0.12782400 \\
$\mathrm{H}$ & 0.70385600 & 2.44223800 & 1.04923000 \\
$\mathrm{H}$ & 0.57826600 & 1.01882700 & 3.34257900 \\
$\mathrm{H}$ & -2.00827800 & 0.41463300 & 3.78673600 \\
$\mathrm{C}$ & -3.70044000 & -2.56037200 & -0.68305700 \\
$\mathrm{H}$ & -4.05517400 & -2.35910400 & -1.69698300 \\
$\mathrm{H}$ & -3.82039100 & -3.62088100 & -0.45534200 \\
$\mathrm{H}$ & -4.26495400 & -1.97335300 & 0.04158600 \\
$\mathrm{C}$ & -1.10574600 & -3.22368000 & -1.71639300 \\
$\mathrm{H}$ & -1.33891000 & -4.26161700 & -1.47270500
\end{tabular}




\begin{tabular}{cccc}
$\mathrm{H}$ & -1.44846400 & -2.98581300 & -2.72655800 \\
$\mathrm{H}$ & -0.02996400 & -3.05895700 & -1.65176000 \\
$\mathrm{C}$ & -1.58916000 & -0.41018200 & -0.77212900 \\
$\mathrm{H}$ & 0.20695500 & -0.65110700 & -1.64794900 \\
$\mathrm{Ir}$ & -1.11765800 & 0.23105100 & 1.05481000 \\
$\mathrm{C}$ & -3.95386500 & 1.01641900 & -1.51744500 \\
$\mathrm{H}$ & -4.38381400 & 1.56365000 & -2.35620600 \\
$\mathrm{H}$ & -3.83854900 & 1.67004200 & -0.65497700 \\
$\mathrm{H}$ & -4.56333400 & 0.14789300 & -1.27501600 \\
$\mathrm{P}$ & 1.74624900 & -0.23609300 & -0.18263400 \\
$\mathrm{C}$ & 2.45910900 & 1.39517900 & -0.62466600 \\
$\mathrm{C}$ & 3.63669900 & 1.87365700 & -0.04611300 \\
$\mathrm{C}$ & 1.70126000 & 2.23882800 & -1.44551600 \\
$\mathrm{C}$ & 4.05873900 & 3.17766800 & -0.29070900 \\
$\mathrm{H}$ & 4.23640600 & 1.22268500 & 0.58369600 \\
$\mathrm{C}$ & 2.13108300 & 3.53919500 & -1.69035200 \\
$\mathrm{H}$ & 0.77399000 & 1.88681700 & -1.89096600 \\
$\mathrm{C}$ & 3.30667500 & 4.01237000 & -1.11201700 \\
$\mathrm{H}$ & 4.98013600 & 3.53820600 & 0.15359600 \\
$\mathrm{H}$ & 1.54264000 & 4.18256000 & -2.33529000 \\
$\mathrm{H}$ & 3.63826900 & 5.02666800 & -1.30484000 \\
$\mathrm{C}$ & 3.24904700 & -1.26327000 & -0.03835800 \\
$\mathrm{C}$ & 3.65407800 & -1.70081300 & 1.22147900 \\
$\mathrm{C}$ & 4.00973100 & -1.59365600 & -1.16368700 \\
$\mathrm{C}$ & 4.82187600 & -2.44786700 & 1.36403200 \\
$\mathrm{H}$ & 3.04874500 & -1.46538600 & 2.09210600 \\
$\mathrm{C}$ & 5.16621900 & -2.34914200 & -1.02178100 \\
$\mathrm{H}$ & 3.68336300 & -1.25941300 & -2.14276000 \\
$\mathrm{C}$ & 5.57582900 & -2.77176500 & 0.24257800 \\
$\mathrm{H}$ & 5.13382100 & -2.78476400 & 2.34631900 \\
$\mathrm{H}$ & 5.75396000 & -2.60699600 & -1.89589900 \\
$\mathrm{H}$ & 6.48154900 & -3.35849900 & 0.34970600 \\
$\mathrm{O}$ & 1.18741200 & -0.74190800 & -1.64267600 \\
\hline & & & \\
\hline
\end{tabular}

Table S25. Cartesian Coordinates of Int1'.

\begin{tabular}{cccc}
\hline Atomic symbol & $\mathrm{x}$ & $\mathrm{y}$ & $\mathrm{z}$ \\
\hline $\mathrm{S}$ & -0.74588200 & -2.40518800 & 0.56527200 \\
$\mathrm{~S}$ & -2.69617300 & 1.09522400 & -1.34409100 \\
$\mathrm{P}$ & -2.24120800 & -1.74131200 & -0.70147800 \\
$\mathrm{O}$ & -3.48962000 & 0.47988200 & -2.42650300 \\
$\mathrm{O}$ & -1.91614900 & 2.31114100 & -1.62235400 \\
$\mathrm{C}$ & -0.86823600 & 0.05398200 & 3.14575400 \\
$\mathrm{C}$ & -1.74286700 & 0.92400900 & 2.45375000 \\
$\mathrm{C}$ & -0.93316300 & 1.90196400 & 1.76633300 \\
$\mathrm{C}$ & 0.43091100 & 1.62739600 & 2.08829800 \\
$\mathrm{C}$ & 0.47937100 & 0.47770100 & 2.94143400 \\
$\mathrm{H}$ & -1.16969700 & -0.83086400 & 3.68748800 \\
$\mathrm{H}$ & -2.81801800 & 0.84039200 & 2.42352400 \\
$\mathrm{H}$ & -1.27320400 & 2.71075100 & 1.13573800 \\
$\mathrm{H}$ & 1.28042100 & 2.19266700 & 1.73301300 \\
$\mathrm{H}$ & 1.36360000 & 0.03882100 & 3.37581300 \\
$\mathrm{C}$ & -3.89081400 & -2.08503800 & -0.00320000
\end{tabular}




\begin{tabular}{|c|c|c|c|}
\hline $\mathrm{H}$ & -4.64830800 & -1.64415800 & -0.65649500 \\
\hline $\mathrm{H}$ & -4.05589000 & -3.16130800 & 0.07288800 \\
\hline $\mathrm{H}$ & -3.95020800 & -1.64275200 & 0.99198900 \\
\hline $\mathrm{C}$ & -2.26002000 & -2.59058300 & -2.30279300 \\
\hline $\mathrm{H}$ & -2.56644500 & -3.63223300 & -2.19128400 \\
\hline $\mathrm{H}$ & -2.95802800 & -2.05383600 & -2.94938800 \\
\hline $\mathrm{H}$ & -1.25894500 & -2.54514500 & -2.73213100 \\
\hline $\mathrm{C}$ & -1.69378900 & -0.11004600 & -0.71124100 \\
\hline $\mathrm{H}$ & -0.34639300 & -0.20790100 & -2.05160000 \\
\hline $\mathrm{Ir}$ & -0.38669200 & -0.01767500 & 0.91324100 \\
\hline $\mathrm{C}$ & -3.94551900 & 1.64646300 & -0.16161000 \\
\hline $\mathrm{H}$ & -4.60404400 & 2.31981600 & -0.70971300 \\
\hline $\mathrm{H}$ & -3.46656200 & 2.17998500 & 0.65595800 \\
\hline $\mathrm{H}$ & -4.50282200 & 0.78217300 & 0.19740700 \\
\hline $\mathrm{P}$ & 1.21427800 & -0.06525400 & -0.63728500 \\
\hline $\mathrm{C}$ & 2.10642400 & 1.51776900 & -0.72601400 \\
\hline $\mathrm{C}$ & 3.42527000 & 1.66520500 & -0.29963100 \\
\hline $\mathrm{C}$ & 1.37566500 & 2.63184500 & -1.15640000 \\
\hline $\mathrm{C}$ & 4.01730000 & 2.92725600 & -0.30081400 \\
\hline $\mathrm{H}$ & 3.99407400 & 0.80156000 & 0.02959700 \\
\hline $\mathrm{C}$ & 1.97714200 & 3.88402500 & -1.16305800 \\
\hline $\mathrm{H}$ & 0.34027200 & 2.52007800 & -1.47335000 \\
\hline $\mathrm{C}$ & 3.29504700 & 4.03380700 & -0.73197300 \\
\hline $\mathrm{H}$ & 5.04379600 & 3.04105600 & 0.02930100 \\
\hline $\mathrm{H}$ & 1.41437200 & 4.74582400 & -1.50366700 \\
\hline $\mathrm{H}$ & 3.75795000 & 5.01447100 & -0.73571900 \\
\hline $\mathrm{C}$ & 2.52194800 & -1.31608100 & -0.50206100 \\
\hline $\mathrm{C}$ & 2.81207300 & -1.92402400 & 0.71716700 \\
\hline $\mathrm{C}$ & 3.27602300 & -1.62968600 & -1.63513000 \\
\hline $\mathrm{C}$ & 3.86136500 & -2.83348700 & 0.80967000 \\
\hline $\mathrm{H}$ & 2.20401600 & -1.69661700 & 1.58686700 \\
\hline $\mathrm{C}$ & 4.31963700 & -2.54257700 & -1.54069900 \\
\hline $\mathrm{H}$ & 3.03568400 & -1.16440100 & -2.58516100 \\
\hline $\mathrm{C}$ & 4.61524600 & -3.14072600 & -0.31805500 \\
\hline $\mathrm{H}$ & 4.08251200 & -3.30976400 & 1.75814900 \\
\hline $\mathrm{H}$ & 4.90169900 & -2.78962300 & -2.42131800 \\
\hline $\mathrm{H}$ & 5.42955400 & -3.85325600 & -0.24722800 \\
\hline $\mathrm{O}$ & 0.64934700 & -0.28501000 & -2.12814800 \\
\hline
\end{tabular}

Table S26. Cartesian Coordinates of TS2'.

\begin{tabular}{cccc}
\hline Atomic symbol & $\mathrm{x}$ & $\mathrm{y}$ & $\mathrm{z}$ \\
\hline $\mathrm{S}$ & -0.70423700 & -2.39600700 & 0.63165100 \\
$\mathrm{~S}$ & -2.73106200 & 1.04401300 & -1.36136700 \\
$\mathrm{P}$ & -2.17642000 & -1.78854500 & -0.67409800 \\
$\mathrm{O}$ & -3.52662100 & 0.39212900 & -2.41621000 \\
$\mathrm{O}$ & -2.00794700 & 2.28357100 & -1.66194600 \\
$\mathrm{C}$ & -0.81237400 & 0.12874600 & 3.15277100 \\
$\mathrm{C}$ & -1.72870700 & 0.96079000 & 2.46568700 \\
$\mathrm{C}$ & -0.95865200 & 1.93826800 & 1.73122400 \\
$\mathrm{C}$ & 0.41961500 & 1.70540800 & 2.02129800 \\
$\mathrm{C}$ & 0.51861300 & 0.57844200 & 2.90073900 \\
$\mathrm{H}$ & -1.07687300 & -0.74829600 & 3.72571500
\end{tabular}




\begin{tabular}{|c|c|c|c|}
\hline $\mathrm{H}$ & -2.80264500 & 0.86088100 & 2.48345400 \\
\hline $\mathrm{H}$ & -1.33076700 & 2.72455800 & 1.09033300 \\
\hline $\mathrm{H}$ & 1.24507500 & 2.28014700 & 1.62708900 \\
\hline $\mathrm{H}$ & 1.42447900 & 0.17084100 & 3.32057800 \\
\hline $\mathrm{C}$ & -3.83508100 & -2.07996000 & 0.01466000 \\
\hline $\mathrm{H}$ & -4.58247500 & -1.65133700 & -0.65755100 \\
\hline $\mathrm{H}$ & -4.00532800 & -3.15324900 & 0.11579300 \\
\hline $\mathrm{H}$ & -3.89786100 & -1.61849500 & 1.00078100 \\
\hline $\mathrm{C}$ & -2.16439200 & -2.66064400 & -2.25851100 \\
\hline $\mathrm{H}$ & -2.41627600 & -3.71443100 & -2.12861800 \\
\hline $\mathrm{H}$ & -2.88948600 & -2.16989300 & -2.91187000 \\
\hline $\mathrm{H}$ & -1.16667500 & -2.56538900 & -2.68817600 \\
\hline $\mathrm{C}$ & -1.62953100 & -0.13288400 & -0.76872900 \\
\hline $\mathrm{H}$ & -0.57637500 & -0.21599200 & -1.78489900 \\
\hline Ir & -0.39117200 & 0.01163500 & 0.92153900 \\
\hline $\mathrm{C}$ & -3.94482300 & 1.51874100 & -0.11693100 \\
\hline $\mathrm{H}$ & -4.67233000 & 2.13430400 & -0.64560900 \\
\hline $\mathrm{H}$ & -3.46304200 & 2.10058100 & 0.66388900 \\
\hline $\mathrm{H}$ & -4.42716000 & 0.62923000 & 0.28413000 \\
\hline $\mathrm{P}$ & 1.21128300 & -0.07002500 & -0.67133500 \\
\hline $\mathrm{C}$ & 2.10083100 & 1.51523000 & -0.77106700 \\
\hline $\mathrm{C}$ & 3.40930000 & 1.68485900 & -0.32215700 \\
\hline $\mathrm{C}$ & 1.37186900 & 2.61303700 & -1.24355400 \\
\hline $\mathrm{C}$ & 3.99103700 & 2.95175600 & -0.34028400 \\
\hline $\mathrm{H}$ & 3.97873500 & 0.83323300 & 0.03656700 \\
\hline $\mathrm{C}$ & 1.96079500 & 3.87105300 & -1.26425100 \\
\hline $\mathrm{H}$ & 0.34650200 & 2.48173300 & -1.58316800 \\
\hline $\mathrm{C}$ & 3.26810800 & 4.04255800 & -0.80931800 \\
\hline $\mathrm{H}$ & 5.01024400 & 3.08202200 & 0.00622800 \\
\hline $\mathrm{H}$ & 1.39804400 & 4.72007600 & -1.63583000 \\
\hline $\mathrm{H}$ & 3.72261900 & 5.02703900 & -0.82537900 \\
\hline $\mathrm{C}$ & 2.51915900 & -1.31798700 & -0.49120400 \\
\hline $\mathrm{C}$ & 2.85906600 & -1.84857800 & 0.75124600 \\
\hline $\mathrm{C}$ & 3.21830100 & -1.71508600 & -1.63261000 \\
\hline $\mathrm{C}$ & 3.90458900 & -2.76064500 & 0.85788600 \\
\hline $\mathrm{H}$ & 2.29159500 & -1.55927100 & 1.63018100 \\
\hline $\mathrm{C}$ & 4.25949100 & -2.63001600 & -1.52436000 \\
\hline $\mathrm{H}$ & 2.93449900 & -1.31147700 & -2.59890200 \\
\hline $\mathrm{C}$ & 4.60559300 & -3.14914800 & -0.27943500 \\
\hline $\mathrm{H}$ & 4.16445500 & -3.17628700 & 1.82501300 \\
\hline $\mathrm{H}$ & 4.79948200 & -2.94069500 & -2.41173700 \\
\hline $\mathrm{H}$ & 5.41705400 & -3.86378600 & -0.19734900 \\
\hline $\mathrm{O}$ & 0.55203500 & -0.30383600 & -2.08194300 \\
\hline
\end{tabular}

Table S27. Cartesian Coordinates of $5^{\prime} \mathrm{Me}$.

\begin{tabular}{cccc}
\hline Atomic symbol & $\mathrm{x}$ & $\mathrm{y}$ & $\mathrm{z}$ \\
\hline $\mathrm{S}$ & 0.79922000 & 2.18602800 & 0.85292300 \\
$\mathrm{~S}$ & 3.29895800 & -0.86532200 & -0.85776200 \\
$\mathrm{P}$ & 1.97699800 & 1.85051600 & -0.78301700 \\
$\mathrm{O}$ & 4.17431000 & -0.23169000 & -1.84812500 \\
O & 2.99692500 & -2.28651400 & -0.99425300 \\
C & 1.33203600 & -1.14569400 & 2.61619500
\end{tabular}


C

C

C

C

$\mathrm{H}$

$\mathrm{H}$

$\mathrm{H}$

$\mathrm{H}$

$\mathrm{H}$

C

$\mathrm{H}$

$\mathrm{H}$

$\mathrm{H}$

C

$\mathrm{H}$

$\mathrm{H}$

$\mathrm{H}$

C

$\mathrm{H}$

Ir

C

$\mathrm{H}$

$\mathrm{H}$

$\mathrm{H}$

$\mathrm{P}$

C

C

C

C

$\mathrm{H}$

C

$\mathrm{H}$

C

$\mathrm{H}$

$\mathrm{H}$

$\mathrm{H}$

C

C

C

C

$\mathrm{H}$

C

$\mathrm{H}$

C

$\mathrm{H}$

$\mathrm{H}$

$\mathrm{H}$

$\mathrm{O}$ $\begin{array}{r}1.17244900 \\ -0.21510800 \\ -0.93063400 \\ 0.02479200 \\ 2.25399400 \\ 1.95183400 \\ -0.65127500 \\ -2.00058900 \\ -0.19369400 \\ 3.66565000 \\ 4.30126700 \\ 3.58834800 \\ 4.08909300 \\ 1.44950500 \\ 1.45040000 \\ 2.16262900 \\ 0.45473000 \\ 1.75851500 \\ 1.32997300 \\ 0.39187900 \\ 4.06797400 \\ 3.99159300 \\ 3.55848000 \\ 5.10999000 \\ -1.21676200 \\ -2.23551600 \\ -3.37483100 \\ -1.79030900 \\ -4.04602800 \\ -3.75475200 \\ -2.46109400 \\ -0.91860300 \\ -3.58630300 \\ -4.93406000 \\ -2.10909700 \\ -4.10975700 \\ -2.45210200 \\ -2.67503600 \\ -3.22782500 \\ -3.67325500 \\ -2.05348800 \\ -4.22361400 \\ -3.03577400 \\ -4.45061400 \\ -3.83751400 \\ -4.82165900 \\ -5.22618200 \\ -0.73584400 \\ \hline\end{array}$

$-2.12912200$

$-2.24991400$

$-1.33163600$

$-0.63708700$

$-0.82949300$

$-2.68254800$

$-2.93407700$

$-1.19428300$

0.15309200

2.48270500

2.18055400

3.57156600

2.13235200

2.64802200

3.72971000

2.37883100

2.28011600

0.04148900

$-0.20774000$

$-0.22430600$

$-0.61859200$

$-1.54865900$

0.18251100

$-0.36652200$

0.20122000

$-1.31840000$

$-1.58067300$

$-2.27235700$

$-2.79404100$

$-0.82963000$

$-3.48278000$

$-2.05533800$

$-3.74835400$

$-2.98871800$

$-4.21799200$

$-4.69207900$

1.42496600

1.72423300

2.05115900

2.62246300

1.26849400

2.95147500

1.82962700

3.23455200

2.85282100

3.43441800

3.93724500

0.61139200
1.59986400

1.28544100

2.13453900

2.91776300

3.07793600

1.09636100

0.57365900

2.15766000

3.62157600

$-0.53390000$

$-1.36610600$

$-0.49040300$

0.40466400

$-2.31446000$

$-2.16790700$

$-3.09802500$

$-2.56788100$

$-0.83890300$

$-1.81596300$

0.74516100

0.75999700

1.31867200

1.29319900

0.57112900

$-0.82406600$

$-1.00201400$

$-0.24143300$

$-1.92028300$

$-0.37668000$

0.44520900

$-2.05587200$

$-2.52980300$

$-1.27837100$

0.21473600

$-2.77110400$

$-1.38431000$

$-0.23281700$

1.11038900

$-1.21025900$

1.47632400

1.87461600

$-0.84591700$

$-2.25513000$

0.49790900

2.52335500

$-1.61092500$

0.78213700

$-2.20464500$ 
Table S28. Cartesian Coordinates of Diphenyl phosphine oxid.

\begin{tabular}{cccc}
\hline Atomic symbol & $\mathrm{x}$ & $\mathrm{y}$ & $\mathrm{z}$ \\
\hline $\mathrm{C}$ & -1.45700100 & 0.34771500 & -0.21789300 \\
$\mathrm{C}$ & -2.05857200 & -0.54670500 & -1.10290300 \\
$\mathrm{C}$ & -2.00253800 & 0.55092900 & 1.04998300 \\
$\mathrm{C}$ & -3.19081900 & -1.25493400 & -0.71302500 \\
$\mathrm{H}$ & -1.65362600 & -0.68445400 & -2.10183200 \\
$\mathrm{C}$ & -3.13524000 & -0.15602800 & 1.43558200 \\
$\mathrm{H}$ & -1.54343500 & 1.27130700 & 1.71936900 \\
$\mathrm{C}$ & -3.72570500 & -1.06057400 & 0.55673000 \\
$\mathrm{H}$ & -3.66057300 & -1.94784500 & -1.40176100 \\
$\mathrm{H}$ & -3.56235300 & 0.00260500 & 2.41918700 \\
$\mathrm{H}$ & -4.61108700 & -1.60859900 & 0.85894900 \\
$\mathrm{C}$ & 1.44015900 & 0.18266600 & -0.22934500 \\
$\mathrm{C}$ & 2.56646700 & 0.79352200 & 0.32064900 \\
$\mathrm{C}$ & 1.42163700 & -1.19781300 & -0.43501500 \\
$\mathrm{C}$ & 3.67662300 & 0.02389600 & 0.65496500 \\
$\mathrm{H}$ & 2.55516900 & 1.86500900 & 0.49087300 \\
$\mathrm{C}$ & 2.53410400 & -1.96178400 & -0.10390400 \\
$\mathrm{H}$ & 0.53736500 & -1.68074500 & -0.83982300 \\
$\mathrm{C}$ & 3.66127600 & -1.35010400 & 0.43988000 \\
$\mathrm{H}$ & 4.55167700 & 0.49714600 & 1.08572100 \\
$\mathrm{H}$ & 2.52005200 & -3.03434600 & -0.26094600 \\
$\mathrm{H}$ & 4.52675700 & -1.94878300 & 0.70122900 \\
$\mathrm{O}$ & 0.16414300 & 2.62042700 & -0.07272200 \\
$\mathrm{P}$ & 0.04289500 & 1.25840700 & -0.66323400 \\
$\mathrm{H}$ & 0.00112300 & 1.20447300 & -2.07489700 \\
\hline & & &
\end{tabular}

Table S29. Cartesian Coordinates of Diphenyl phosphinous acid.

\begin{tabular}{cccc}
\hline Atomic symbol & $\mathrm{X}$ & $\mathrm{y}$ & $\mathrm{z}$ \\
\hline $\mathrm{C}$ & -1.39917900 & 0.37434600 & -0.22370900 \\
$\mathrm{C}$ & -2.32051200 & -0.13193800 & -1.13838500 \\
$\mathrm{C}$ & -1.55225100 & 0.09382300 & 1.13696000 \\
$\mathrm{C}$ & -3.37967000 & -0.92750700 & -0.70310100 \\
$\mathrm{H}$ & -2.21582700 & 0.10025000 & -2.19437600 \\
$\mathrm{C}$ & -2.61517900 & -0.68526500 & 1.57183000 \\
$\mathrm{H}$ & -0.83619400 & 0.49591100 & 1.84676400 \\
$\mathrm{C}$ & -3.52707600 & -1.20134700 & 0.65063100 \\
$\mathrm{H}$ & -4.09152700 & -1.32201600 & -1.41961700 \\
$\mathrm{H}$ & -2.73349900 & -0.89872300 & 2.62849400 \\
$\mathrm{H}$ & -4.35372200 & -1.81408000 & 0.99295700 \\
$\mathrm{C}$ & 1.37504500 & 0.27108900 & -0.34135900 \\
$\mathrm{C}$ & 2.37185500 & 0.68061100 & 0.54282300 \\
$\mathrm{C}$ & 1.44521300 & -1.00245300 & -0.91312100 \\
$\mathrm{C}$ & 3.42264600 & -0.17937200 & 0.85577200 \\
$\mathrm{H}$ & 2.31435200 & 1.66498200 & 0.99172400 \\
$\mathrm{C}$ & 2.49418200 & -1.85644300 & -0.60031600 \\
$\mathrm{H}$ & 0.66852400 & -1.33463000 & -1.59703600 \\
$\mathrm{C}$ & 3.48761800 & -1.44513600 & 0.28591300 \\
$\mathrm{H}$ & 4.19141300 & 0.14340000 & 1.54942600
\end{tabular}




\begin{tabular}{lccc}
$\mathrm{H}$ & 2.53642800 & -2.84463200 & -1.04474900 \\
$\mathrm{H}$ & 4.30704000 & -2.11157600 & 0.53087200 \\
$\mathrm{O}$ & 0.17295700 & 2.47521500 & 0.42189200 \\
$\mathrm{P}$ & 0.00595200 & 1.38713600 & -0.83853800 \\
$\mathrm{H}$ & -0.07608400 & 3.36990500 & 0.17484900 \\
\hline
\end{tabular}

